\title{
A Secure and Privacy-Friendly IP-based Emergency Services Architecture
}

\author{
Dissertation \\ for the award of the degree \\ 'Doctor rerum naturalium' \\ at the Georg-August-Universität Göttingen \\ within the doctoral programme/doctoral degree programme \\ of the Georg-August University School of Science (GAUSS)
}

submitted by

Hannes Tschofenig

from Villach, Austria

Göttingen, November 2019 
Thesis advisory committee

Prof. Dr. Xiaoming Fu, Georg-August Universität Göttingen

Prof. Dr. Dieter Hogrefe, Georg-August Universität Göttingen

Members of the examination board

Referee: Prof. Dr. Xiaoming Fu, Georg-August Universität Göttingen

Co-referee: Prof. Dr. Dieter Hogrefe, Georg-August Universität Göttingen

Co-referee: Prof. Dr. Thomas Schmidt, HAW Hamburg

Other members of the Examination Board

Prof. Dr.-Ing. Marcus Baum, Georg-August Universität Göttingen

Prof. Dr. Ramin Yahyapour, Georg-August Universität Göttingen

Prof. Dr.-Ing. Delphine Reinhardt, Georg-August Universität Göttingen

Day of the oral examination: 28. November 2019 


\begin{abstract}
Emergency services support is one of the most valued features of the public switched telephone network. With the transition to IP-based communication the opportunity to re-design the emergency services communication architecture emerged. It was a chance to offer more features with improved security and privacy properties.
\end{abstract}

This dissertation makes a contribution to the design of an IP-based emergency services architecture that offers international applicability, and multi-media support. Due to the extended functionality emergency calls can also be triggered by vehicles and Internet of Things devices. Security and privacy was taken care off during the work on this architecture. 



\section{Zusammenfassung}

Die Unterstützung von Notrufdiensten ist eine der am meisten geschätzten Funktionen des öffentlichen Telefonnetzes. Mit dem Übergang zur IP-basierten Kommunikation werden neue Geschäftsmodelle und Funktionen untersucht. Diese Entwicklung bietet die Möglichkeit einer Neugestaltung, die unter anderem auch sicherer ist und bessere Eigenschaften für den Datenschutz bietet.

Diese Dissertation leistet einen Beitrag zur Entwicklung einer auf dem Internet Protokoll basierenden Notrufarchitektur, welche international anwendbar ist und eine Multimediafähigkeit besitzt. Durch die erweiterte Funktionalität können Notrufe auch von Fahrzeugen und von Internet der Dinge ausgelöst werden. Auf Sicherheit und Datenschutz wurde schon bei der Entwicklung der Architektur Rücksicht genommen. 



\section{Acknowledgements}

I would like to thank my supervisor Prof. Dr. Xiaoming Fu for inviting me to work in his group, and for the support he provided to me over the years. During that time we contributed to the research community and also to standards developing organizations. I would also like to thank Prof. Dr. Dieter Hogrefe for his guidance regarding my $\mathrm{PhD}$ thesis. My gratitude also goes to all members of the examination board.

During the work on my thesis I met many researchers, product developers, standardization experts, practitionaires from emergency services authorities, regulators, politicians, and even those who benefitted from the existence of the emergency services infrastructure. In summary, I was fortunate to get to know so many great people who dedicated their life to improve the emergency services infrastructure to make a difference for others.

In particular, I would like to thank those who worked with me in the Internet Engineering Task Force (IETF), the European Emergency Number Association (EENA), and the National Emergency Number Association (NENA).

From February 2005 to March 2010 I co-chaired the IETF Emergency Context Resolution with Internet Technologies (ECRIT) working group, which developed many of the standards described in this thesis. I particlularly would like to thank Brian Rosen, Marc Linsner, Henning Schulzrinne, Roger Marshall, Randy Gellens, Alissa Cooper, James Winterbottom, Laura Liess, Tom Taylor, Ted Hardie, Andy Newton, James Polk, Martin Thomson, Karl Heinz Wolf, Barbara Stark, Andres Kuett, Christer Holmberg, Milan Patel, Bernard Aboba, Stephen McCann, Gabor Bajko, Dirk Kroeselberg, Jon Peterson, Jonathan Rosenberg, Alexander Mayrhofer, Richard Barnes, Mary Barnes, John Morris, Matt Lepinski, Rohan Mahy, and Ray Bellis. Many of the above-mentioned individuals have also contributed to the work on location technologies in the IETF Geolocation and Privacy (GEOPRIV) working group.

I have to thank Jon Peterson and Allison Mankin for appointing me to the co-chair of the ECRIT working group together with Marc Linsner after a very successful Birds of a Feather (BOF) in November 2004 at the 61st IETF meeting in Washington DC/USA.

Soon after we started our work in the IETF ECRIT working group we had to realize that other organizations also began to standardize emergency services functionality. Many in the ECRIT working group were convinced that these ongoing activities had to be harmonized to ensure the best possible interoperability. This lead to a series of emergency services 
workshops, which helped to exchange information about ongoing activities and to make members of various organizations to get to know each other better.

During one of the first workshops I met Paul-Morandini and Gary Machado from EENA and also the leadership, in particular Roger Hixson, from NENA. Their view about emergency services was much wider than our purely technical focus in the IETF ECRIT working group. Over the years many standardization experts got involved in the work in EENA and NENA. We all better understood the complexity of the regulatory frameworks and the bigger emergency services ecosystem. It was in February 2009 when I was awarded for the 'Outstanding Vision for 112' from EENA and later became the co-chair of the NG112 technical committee and an EENA board member.

I would like to particulary thank the members of the EENA advisory board, the members of the EENA NG112 Technical Committee, and the members of the NENA long-term definition and the additional data working groups: Gary Machado, Cristina Lumbreras, Tony O’Brien, Wolfgang Kampichler, Jerome Paris, Emmanuel Paul, Greg Rohde, John Medland, Tadas Maroscikas, Gerhard Fischer, Peter Woodford, Yacine Rebahi, Mark Fletcher, Anand Akundi, Carla Anderson, John Chiaramonte, Gordon Vanauken, Byron Smith, Jakob Schlyter, and Gunnar Hellstroem.

I would also like to thank those who have contributed to standardization efforts from other organizations, such as the Open Geospatial Consortium (OGC), the European Telecommunications Standards Institute (ETSI), the Open Mobile Alliance (OMA), and the 3rd Generation Partnership Program (3GPP): Ed Wells, Carl Reed, Khiem Tran, Jan Kall, Hannu Hietalahti, Chantal Bonardi, Roger Hixson, Matt Serra, Martin Dawson, and Guy Caron.

Many persons have contributed to make the emergency services workshop series a success with their organization and their contributions. Many of the participants have later contributed to the IP-based emergency services book, which Henning Schulzrinne and I co-edited, to help capture the state-of-the-art so that others can contribute to emergency services activities more easily.

I would also like to thank Krzysztof Rzecki, Piotr Blaszczyk, Anna Makarowska, and Michal Niedzwiecki for their work on a proof-of-concept implementation of various emergency services protocols described in this thesis. Their implementation support has helped to improve the quality of the LoST specification, and to gain insight into performance and feasibility. The LoST implementation has also been used during interoperability events and it can be downloaded from http://ecrit. sourceforge.net. Furthermore, I would like to thank Muraguaj Shanmugam, and Mayutan Arumaithurai for their help with implementations. 
I am grateful that I met Henning Schulzrinne in the early days of my career. He taught me to work more efficiently, we developed many technical solutions together, and organized several workshops.

I would also like to extend my gratitude to the current and former members of the Telematics Group at the University of Göttingen for their help, particularly Youssef El Hajj Shehadeh, Muraguaj Shanmugam, and Mayutan Arumaithurai.

I am very thankful to the administrative support from the University of Goettingen. I would like to thank Annette Kadziora, Nina Giebel, Prof. Dr. Jens Grabowski, Prof. Dr. Stephan Waack, and Federica Poltronieri for their help. 



\section{Contents}

Table of Contents $\quad$ xi

List of Figures $\quad$ xv

List of Tables

Acronyms $\quad$ xvii

1. Introduction 1

1.1. Background ...................... 1

1.2. Research Challenges . . . . . . . . . . . . . . . . . 2

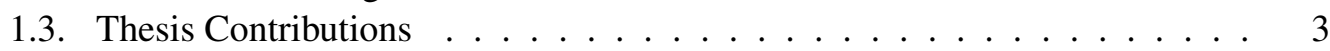

1.4. Thesis Organization . . . . . . . . . . . . . . 5

1.5. Standards Contributions . . . . . . . . . . . . . . . . 6

2. Requirements 11

3. Related Work 15

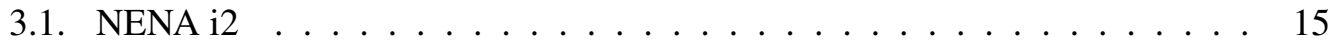

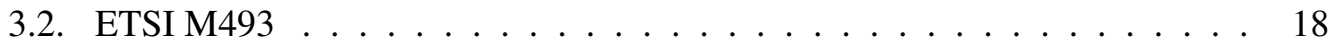

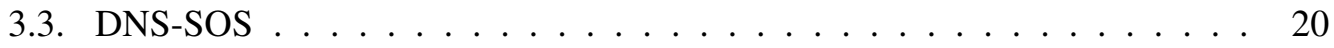

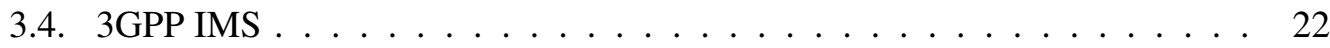

4. Comparison 25

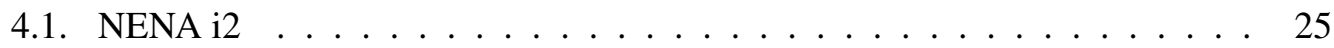

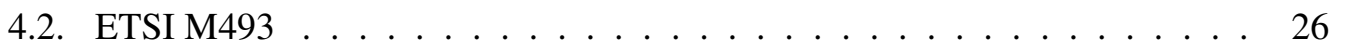

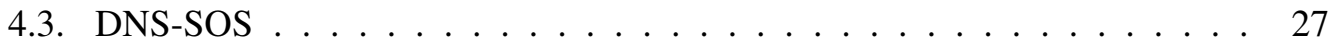

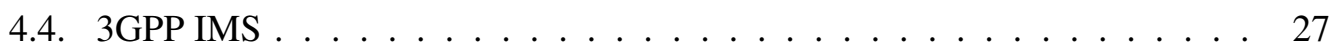

5. An IP-based Emergency Services Architecture 31

5.1. Building Blocks . . . . . . . . . . . . . . . . . . 34

5.2. Routing Emergency Calls . . . . . . . . . . . . . . . . . . . 36

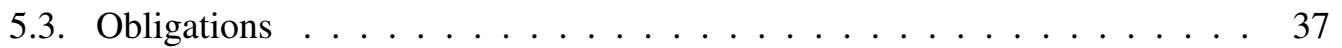

5.3.1. End Hosts . . . . . . . . . . . . . . . . . . . . . . . 38 


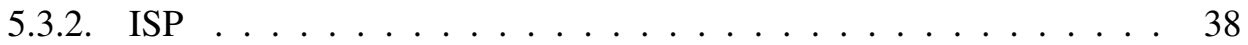

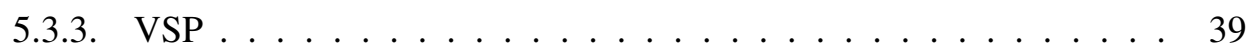

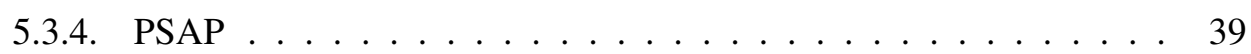

5.4. LoST Mapping Architecture . . . . . . . . . . . . . . . . . . 40

5.5. Steps towards an IETF Emergency Services Architecture . . . . . . . . . . 44

5.5.1. Legacy End Points . . . . . . . . . . . . . . . . . . . 44

5.5.2. Partially Upgraded End Hosts . . . . . . . . . . . . . . . . 46

5.5.3. Emergency Services for Persons with Disabilities . . . . . . . . . 46

5.5.4. Vehicle Initiated Emergency Calls . . . . . . . . . . . . . 47

5.5.5. Additional Data . . . . . . . . . . . . . . . . . . . 48

5.5.6. Data-Only Emergency Calls . . . . . . . . . . . . . . . . . 49

6. Securing IP-based Emergency Services Networks 51

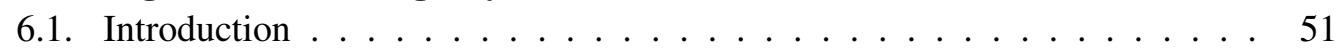

6.2. Communication Model . . . . . . . . . . . . . . . . . . 52

6.3. Adversary Models . . . . . . . . . . . . . . . . . . . . . . . . . . . . . . . . . . . . . 54

6.4. Security Threats . . . . . . . . . . . . . . . . . . 55

6.4.1. Denial of Service Attacks . . . . . . . . . . . . . 56

6.4.2. Attacks Involving the Emergency Identifier . . . . . . . . . . . . . 57

6.4.3. Attacks Against the Mapping System _. . . . . . . . . . . . 58

6.4.4. Attacks against the Location Information Server . . . . . . . . 60

6.4.5. Swatting . . . . . . . . . . . . . . . . . . 61

6.4.6. Attacks to Prevent a Specific Individual from Receiving Aid . . . . 62

6.4.7. Attacks to Gain Information about an Emergency . . . . . . . . . 63

6.4.8. Interfering with the LIS and LoST Server Discovery Procedure . . . 64

6.4.9. Call Identity Spoofing . . . . . . . . . . . . . . . . . 64

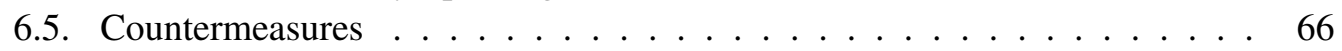

6.5.1. Discovery . . . . . . . . . . . . . . . . . 66

6.5.2. Secure Session Setup and Caller Identity . . . . . . . . . . . . . 69

6.5.3. Media Exchange . . . . . . . . . . . . . . . . . 71

6.5.4. Mapping Database Security . . . . . . . . . . . . 72

7. Securing Data-Only Emergency Calls $\quad \mathbf{7 3}$

7.1. Introduction . . . . . . . . . . . . . . . . . 73

7.2. Deployment Scenarios . . . . . . . . . . . . . . . . . . . . 75

7.3. Security and Privacy Threats . . . . . . . . . . . . . . 76

7.3.1. Categories of Attacks . . . . . . . . . . . . . . . . 76

7.3.2. Threat Modelling . . . . . . . . . . . . . . . . . . . 77

7.4. Overview of ENISA Guidelines . . . . . . . . . . . . . . 78

7.5. IETF IoT Security Standardization . . . . . . . . . . . . . . . 79

7.5.1. Authentication and Communication Security . . . . . . . . . . 79 
7.5.2. Object Security . . . . . . . . . . . . . . . . . . 82

7.5.3. Authorization and Access Control . . . . . . . . . . . . . . . . 84

7.5.4. Key Management . . . . . . . . . . . . . . . . . . . . . 86

7.5.5. State-of-the-Art Crypto . . . . . . . . . . . . . . . . . 87

7.5.6. Restricting Communication . . . . . . . . . . . . . . . 89

7.5.7. Firmware and Software Updates . . . . . . . . . . . . . . 90

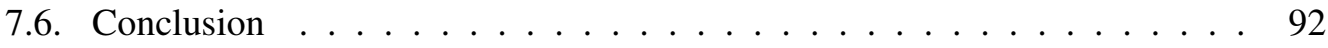

8. Conclusion 95

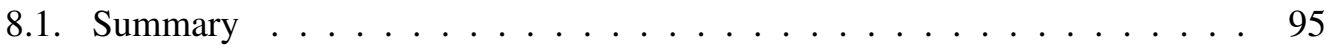

8.2. Future Work . . . . . . . . . . . . . . . . . . 96

$\begin{array}{lr}\text { Software } & 98\end{array}$

A. LoST Software 99

A.1. LoST Client . . . . . . . . . . . . . . . . . . . . . . . . . . . 99

A.2. LoST Server . . . . . . . . . . . . . . . . . . . . . 102

A.3. Database . . . . . . . . . . . . . . . . . 106

A.4. Performance Analysis . . . . . . . . . . . . . . . . . . . 109

Bibliography 122 



\section{List of Figures}

1.1. Thesis Organization. $\ldots \ldots \ldots \ldots \ldots \ldots$

3.1. NENA i2 Architecture (Simplified) . . . . . . . . . . . . . . . . . . 16

3.2. M493 Architecture (Simplified) . . . . . . . . . . . . . . . . . . . . 19

3.3. Example DNS-SOS Configuration. . . . . . . . . . . . . . . . 22

3.4. IMS Architecture (Simplified) . . . . . . . . . . . . . . . . . . . 24

5.1. Communication Architecture Overview . . . . . . . . . . . . 32

5.2. High-Level Functionality of Location-to-Service Translation (LoST). . . . . 37

5.3. Main Components involved in an Emergency Call. . . . . . . . . . . . . . 37

5.4. Trees and Forest Guides in the LoST Mapping Architecture. . . . . . . . . 41

5.5. Example Query / Response in the LoST Mapping Architecture. . . . . . . . 43

5.6. Mapping Element. . . . . . . . . . . . . . . . . . . . . . . . 43

5.7. Emergency Services Architecture with Legacy End Points. . . . . . . . . . 45

6.1. Communication Model Overview. . . . . . . . . . . . . . . . . . 53

7.1. IoT Deployment Scenarios. . . . . . . . . . . . . . . . . . . . . . 75

7.2. Categories of Threats. . . . . . . . . . . . . . . . 76

A.1. Database Design. . . . . . . . . . . . . . . . . . . 108

A.2. Plot of the U.S. TIGER County Dataset (2018). . . . . . . . . . . . . 110

A.3. Example data to be inserted in LoST Database. . . . . . . . . . . . . 113

A.4. Plot of example queries. . . . . . . . . . . . . . . . . . 118

A.5. CSV file with example queries. . . . . . . . . . . . . . . . . . . . . 119

A.6. Roundtrip Time of findService Request/Response. . . . . . . . . . . . . . . 121 



\section{List of Tables}

4.1. Emergency Services Architecture Solution Comparison. . . . . . . . . . . . 29 



\section{Acronyms}

3G 3rd Generation (Mobile Systems)

3GPP 3rd Generation Partnership Program

AAA Authentication, Authorization and Accounting

ALI Automatic Location Identification

AN Access Network

ASP Application Service Provider

CA Certification Authority (often also called Certificate Authority)

CAP Common Alerting Protocol

CS Circuit Switched

CSCF Call Session Control Function

CSP Communication Service Provider

DHCP Dynamic Host Configuration Protocol

DNS Domain Name Server (or Service or System)

DoS Denial of Service

DSL Digital Subscriber Line

EAP Extensible Authentication Protocol

ECRIT Emergency Context Resolution with Internet Technologies

E-CSCF Emergency Call Session Control Function

EENA European Emergency Number Association

ERDB Emergency Service Zone Routing Data Base

ERT Emergency Route Tuple

ESGW Emergency Services Gateway

ESGWRI Emergency Services Gateway Route Identifier

ESINet Emergency Services IP Network

ESN Emergency Service Number, Emergency Service Network

ESNet Emergency Services Network

ESQK Emergency Services Query Key

ESRD Emergency Services Routing Digits

ESRK Emergency Services Routing Key 
ESRP Emergency Services Routing Proxy

ESZ Emergency Services Zone

ETSI European Telecommunications Standards Institute (ETSI)

FCC Federal Communications Commission

Geopriv Geolocation and Privacy

GML Geographic Markup Language

GPRS General Packet Radio Service

GPS Global Positioning System

GSM Global Standard for Mobile Communication

HELD HTTP Enabled Location Delivery

HSS Home Subscriber Server

HTTP Hypertext Transfer Protocol

IANA Internet Assigned Numbers Authority

IAP Internet Access Provider

I-CSCF Interrogating Call Session Control Function

IEEE Institute of Electrical and Electronics Engineers

IETF Internet Engineering Task Force

ILP Internal Location Protocol

IM Instant Messaging

IMEI International Mobile Equipment Identity

IMS IP Multimedia Subsystem

IMSI International Mobile Subscriber Identity

IoT Internet of Things

IP Internet Protocol

IPsec Internet Protocol Security

ISP Internet Service Provider

IVS In-Vehicle System

LBS Location Based Services

LbyR Location by Reference

LbyV Location by Value

LCP Location Configuration Protocol

LCS Location Service

LIS Location Information Server

LO Location Object

LoST Location to Service Translation 
LRF Location Retrieval Function

LRO Last Routing Option

LS Location Server

LTD Long Term Definition

LTE Long Term Evolution

LVF Location Validation Function

MAC Media Access Control

ME Mobile Equipment

MLP Mobile Location Protocol

MLS Mobile Location Service

MS Mobile Station

MSAG Master Street Address Guide

MSD Minimum Set of Data

MSISDN Mobile Station International ISDN Number

MSRP Message Session Relay Protocol

NAD83 North American Datum 1983

NAI Network Access Identifier

NAP Network Access Provider

NAPTR Naming Authority Pointer

NAT Network Address Translation

NAVD88 North American Vertical Datum of 1988

NENA National Emergency Number Association

NG9-1-1 Next Generation 9-1-1

NGO Non-Governmental Organization

NRA National Regulatory Authority

NSP Network Service Provider

OGC Open Geospatial Consortium

OMA Open Mobile Alliance

PAI P-Asserted-Identity

P-CSCF Proxy Call Session Control Function

Phone Either ME or UE

PIDF Presence Information Data Format

PIDF-LO Presence Information Data Format - Location Object

PKI Public Key Infrastructure

PLMN Public Land Mobile Network 
PS Packet Switched

PSAP Public Safety Answering Point

PSK-TLS Pre-shared Key TLS

PSTN Public Switched Telephone Network

QoP Quality of Position

QoS Quality-of-Service

RADIUS Remote Access Dial-In User Service

RDF Routing Determination Function

RDS Root Discovery Server

RFC Request For Comment

RTCP Real Time Control Protocol

RTP Real Time Transport Protocol

RTSP Real Time Streaming Protocol

RTT Real Time Text

SBC Session Border Control

S-CSCF Serving Call Session Control Function

SDES Session Description Protocol Security Descriptions

SDO Standards Development Organization

SDP Session Description Protocol

SET SUPL Enabled Terminal

SGSN Serving GPRS Support Node

SIM Subscriber Identity Module

SIP Session Initiation Protocol

SMS Short Message Service

SRDB Selective Routing Database

SRTP Secure Real Time Transport Protocol

SSAC Service Specific Access Control

STA Station (an entity with 802.11 interface)

SUPL Secure User Plane Location

TCP Transmission Control Protocol

TDD Telecommunications Device for the Deaf and Hard-of-Hearing

TLS Transport Layer Security

TSP Telematics Service Provider

TTY Teletypewriter (a.k.a. TDD)

UA User Agent 
UAC User Agent Client

UAS User Agent Server

UDP User Datagram Protocol

UE User Equipment

U-NAPTR Straightforward URI-Enabled NAPTR

URI Uniform Resource Identifier

URL Uniform Resource Locator

URN Uniform Resource Name

USI Universal Services Interface

VDB Validation Database

VEDS Vehicle Emergency Data Set

VolP Voice over IP

VPC VoIP Positioning Center

VPN Virtual Private Network

VSP VoIP Service Provider

WebRTC Web Real-Time Communication

WiFi WLAN based on IEEE802.11

XML eXtensible Markup Language

XMPP eXtensible Messaging and Presence Protocol 



\section{Chapter 1}

\section{Introduction}

\subsection{Background}

The ability to call up the police, the fire department or an ambulance in emergencies is one of the most important functions available when using the telephone. As telephone functionality moves from circuit-switched to Internet telephony, its users rightfully expect that this core feature will continue to be available and work as well as it has in the past. Users also expect to be able to reach emergency assistance using new communication devices, such as instant messaging or real-time text, and utilize new media, such as video or data. In all cases, the basic objective is the same: The person seeking help needs to be connected with the most appropriate public safety answering point (PSAP), where call takers dispatch assistance to the caller's location. Users typically assume accurate location to be available to call takers and first responders. PSAPs are responsible for a particular geographic region, which can be as small as a single university campus or as large as a country.

The Internet infrastructure is used for a wide range of services, including for real-time communication between friends, within enterprises, and with business partners as part of e-commerce transactions. IP-based emergency services are an extension of this communication infrastructure. Around 2004/2005, work on IP-based emergency services was kicked off officially by the Internet Engineering Task Force (IETF) with the formation of the 'Emergency Context Resolution with Internet Technologies' (ECRIT) working group. At that time the standardization work on the VoIP-based communication protocol SIP (the Session Initiation Protocol) was ongoing, and it took many more years to get all the necessary building blocks standardized. The foundational work on the SIP protocol was important for the work in ECRIT because telephony providers wanted to use a standardized protocol they could rely on and use as a replacement for their circuit-switched telephony technology. This shift from circuit-switched networks to IP-based VoIP services took place for several reasons, 
including:

1. Lower capital expenses due to the commodity nature and increased competition,

2. Lower operational expenses of IP-based VoIP infrastructure,

3. Future-proofing because the rest of the industry moved to IP as well, and

4. Increased functionality and better extensibility.

The transition to IP-based networking and VoIP allows an emergency services authorities to connect PSAPs with each other and to reroute calls. Rerouting can happen for various reasons, including load balancing, better utilizing call taker skills (e.g., language skills), rerouting incorrectly routed calls, use of conference bridging capabilities, distributing technical functionality (e.g., separating the handling of vehicular emergency calls from emergency calls using instant messaging and real-time text), etc. Furthermore, multimedia data from end devices as well as from service providers can be distributed to entities along the emergency services chain, such as first responders, to improve situational awareness.

The transition to an all-IP-based emergency services network is also used to rethink the established emergency services organization. As a result, the existing processes and organizational structures were simplified, typically leading to a reduction in the number of PSAPs while accomplishing the same mission.

\subsection{Research Challenges}

At the time work on this thesis began, IP-based emergency services were at an early state of deployment. Proprietary and standardized voice-over-IP solutions were available and deployed but they typically did not offer emergency services capabilities ${ }^{1}$. If emergency services support was provided by a VoIP service provider, it was through interconnection with the public switched telephone network (PSTN) because none of the PSAPs were IP capable at that time.

The requirements for an IP-based emergency services architecture had been finalized in the IETF ECRIT working group, with contributions from a wide range of stakeholders when this thesis was started. Due to the differences between the VoIP and the circuit-switched architecture, it was clear that an IP-based emergency services architecture would have to be redesigned; just copying the concepts from the circuit-switched world was not possible.

\footnotetext{
${ }^{1}$ Emergency services support, at a minimum, means to have the possibility to dial an emergency number to reach a call taker at a Public Safety Answering Point (PSAP).
} 
First, an IP-based emergency services architecture had to be designed. As part of this architecture, a protocol for mapping service identifiers and geodetic or civic location information to service contact URIs had to be developed. This protocol had to be flexible enough to work in different deployment scenarios while also offering performance to be used in an emergency services context. There was a question about feasibility and whether the performance expectations could be met.

Second, security and privacy had to be considered during the design because emergency services are critical infrastructure. With an increasing amount of attacks against IP-based services, there was the concern that the transition to IP-based emergency services would make the new infrastructure more vulnerable and fragile.

Third, multimedia-based emergency calling had to be supported. In addition to voice, video, instant messaging, and real-time text, more advanced forms of emergency services also had to be offered. The concept of a data-only emergency call was born with the introduction of emergency services triggered by Internet of Things (IoT) devices, including vehicles. Special attention had to be paid to the security of IoT devices interacting with emergency services.

\subsection{Thesis Contributions}

This dissertation discusses an IP-based emergency services architecture "to ensure that everyone has access to emergency services anytime, anywhere, from any device"2.

The contributions of this dissertation are threefold:

1. The IP-based emergency services architecture required a robust way to route emergency calls to the appropriate PSAPs. The Location-to-Service Translation (LoST) protocol was developed to provide this functionality. I am a co-author of the LoST technical specification, which has been verified with an implementation. The LoST specification has been published in RFC 5222. Details about the implementation including a performance analysis can be found in Appendix A. The performance analysis illustrated that the LoST protocol can be used on devices without negatively impacting emergency services dispatch in terms of latency for call setup.

2. Improving the security properties of the architecture was important, which lead to the

\footnotetext{
${ }^{2}$ Access to emergency services anytime, anywhere and from any device is the stated goal of the National Emergency Number Association (NENA), an emergency services association with more than 9,000 members in 48 chapters across the United States and around the globe [1].
} 
development of various technical specifications and the publication of research papers on the security and privacy of the developed IP-based emergency services architecture. Security mechanisms have been added to the standardized protocols to the greatest extent possible, while the consequences of certain attacks, such as DDoS attacks against first responders, have been highlighted.

3. As the field of emergency services has been expanded to support more than just voice with the addition of data-only emergency services capabilities, I have contributed several technical specifications on Internet of Things security. An initial survey of standardized IoT security protocols has been published in [2], and has recently been updated [3]. The latest survey concludes that "standardization work has advanced to a point that there is typically no need for homegrown solutions". The article does, however, also acknowledge that implementations lag behind standardization and some standardization efforts are still ongoing. The lack of a firmware update mechanism for low-end IoT devices has been a security challenge in many IoT deployments. Firmware update mechanism allows for upgrades to these devices with new emergency services features and protocols. In [4] we asked ourselves whether it is possible to create a secure, standards-compliant firmware update solution that uses state-of-the-art crypto for low-end IoT devices. While this is one of the currently ongoing standardization efforts, the article concludes that incorporating a secure firmware update solution is possible with IoT devices that have as little as $32 \mathrm{kB}$ of RAM and $128 \mathrm{kB}$ of flash memory. Such low-end devices would, however, not offer multi-media support but rather data-only functionality.

The contributions of this dissertation have been published in the following articles:

1. H. Schulzrinne, H. Tschofenig, A. Newton, and T. Hardie, LoST: A Protocol FOR Mapping Geographic Locations to Public Safety Answering Points, Proceedings of the 26th IEEE International Performance Computing and Communications Conference, IPCCC 2007, April 11-13, 2007, New Orleans, Louisiana.

2. H. Tschofenig, H. Schulzrinne, M. Shanmugam, and A. Newton, Protecting FirstLevel Responder Resources in AN IP-BAsed EMERgency SERvices ARCHITECTURE, Proceedings of the 26th IEEE International Performance Computing and Communications Conference, IPCCC 2007, April 11-13, 2007, New Orleans, Louisiana.

3. H. Tschofenig, M. Arumaithurai, H. Schulzrinne, and B. Aboba, How SECURE IS THE Next Generation of IP-based Emergency Services Architecture?, International Journal of Critical Infrastructure Protection (Elsevier), Volume 3, Issue 1, Pages 41-50, May 2010.

4. S. Keoh, S. Kumar, and H. Tschofenig, Securing the Internet of Things: A StANDARdizATION PERSPECTIVE, IEEE Internet of Things Journal, June 2014.

5. H. Tschofenig, and E. Baccelli, Cyber-Physical Security for the Masses: SurVEy of The IP Protocol Suite for IoT Security, IEEE IoT Security \& Privacy, 
Volume: 17 , Issue: 5 , Sept.-Oct. 2019.

\subsection{Thesis Organization}

The work on the IETF emergency services architecture evolved as shown in Figure 1.1, which is also how this dissertation is organized ${ }^{3}$. From a set of requirements an architecture was derived and a solution design developed. The solution consists of functionality for multi-media emergency calls, vehicular emergency calls and data-only emergency calls. Mechanisms for ensuring proper security and privacy properties cut accross the design of all mechanisms.

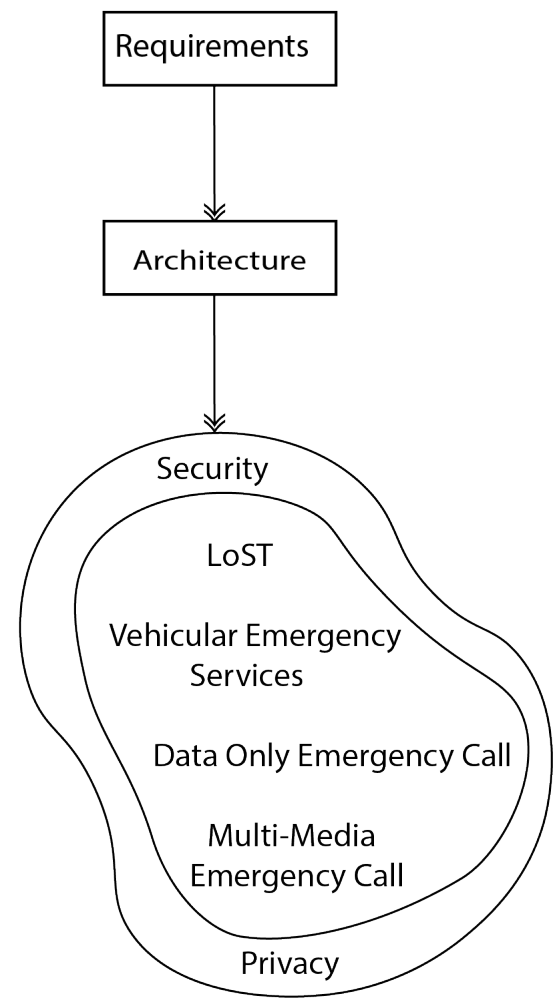

Figure 1.1.: Thesis Organization.

Chapter 2 gives a high-level overview of the requirements and points to requirements

${ }^{3}$ Chapter 5 , Chapter 6 and Chapter 7 are based on publicatications listed above. The text of the publications had been modified to accomodate the different focus. 
documents published by the IETF ECRIT group. These requirements served as the starting point for this thesis. The section concludes with a description of what is outside the scope of this thesis because the field of emergency services is huge.

Chapter 5 describes the IETF emergency services architecture, which was developed based on the requirements in Chapter 2. This chapter also provides an overview of the solution components and explains how the Location to Service Translation (LoST) protocol fits into this architecture. LoST is a key building block for routing emergency calls in the IETF emergency services architecture and Appendix A provides details on the implementation and a performance analysis.

Chapter 6 explores security and privacy aspects of the architecture described in Chapter 5 and explains what security mitigation techniques are available.

Chapter 7 surveys ways to secure IoT devices used in data-only emergency calls. The resulting survey has applicability beyond the field of emergency services. With the advances in standardization and research more technologies are available not only for communication security but also for securing the lifecycle of IoT devices, including firmware updates.

Other solution designs for an IP-based emergency services architecture have been developed as well. Chapter 3 describes those alternative architectures, namely NENA i2, ETSI M493, DNS-SOS, and the 3GPP IMS. While these architectures re-use some of the technical building blocks developed during this thesis they utilize them in a different arrangement. Chapter 4 compares the IETF emergency services architecture against these alternatives.

Chapter 8 summarizes this thesis and discusses future work.

\subsection{Standards Contributions}

A functioning emergency services system requires standardized protocols and procedures. During the work on this thesis I have co-authored the following technical specifications:

1. B. Rosen, H. Schulzrinne, H. Tschofenig, and R. Gellens, DATA-Only EMERGENCY CALLS, draft-ietf-ecrit-data-only-ea-18 (work in progress), Apr. 2019.

2. R. Gellens, and H. Tschofenig, Next-Generation Pan-European eCall, IETF RFC 8147 (Standards Track), May 2017.

3. R. Gellens, B. Rosen, and H. Tschofenig, Next-Generation Vehicle-Initiated EMERGENCY CALLS, IETF RFC 8148 (Standards Track), May 2017. 
4. R. Gellens, B. Rosen, H. Tschofenig, R. Marshall, and J. Winterbottom, AdDitionaL Data Related to AN EMERGENCy CALl, IETF RFC 7852 (Standards Track), July 2016.

5. J. Winterbottom, H. Tschofenig, and L. Liess, A Routing REQuest EXTENSION FOR THE HTTP-EnAbled Location Delivery (HELD) Protocol, IETF RFC 7840 (Standards Track), May 2016.

6. H. Schulzrinne, S. McCann, G. Bajko, H. Tschofenig, and D. Kroeselberg, ExTEnsions to the Emergency Services Architecture for Dealing With UnauthenTICATED AND UnAUTHORIZED DEVICES, IETF RFC 7406 (Informational), Dec. 2014.

7. H. Tschofenig, H. Schulzrinne, B. Aboba, Trustworthy Location, IETF RFC 7378 (Informational), Dec. 2014.

8. J. Peterson, H. Schulzrinne, H. Tschofenig, Secure Telephone Identity Problem STATEMENT AND REQuiREMENTS, IETF RFC 7340 (Informational), Sep. 2014.

9. H. Schulzrinne, H. Tschofenig, C. Holmberg, and M. Patel, PubliC SAFETy AnswerING Point (PSAP) CALlBACK, IETF RFC 7090 (Standards Track), Apr. 2014.

10. R. Barnes, M. Thomson, J. Winterbottom, and H. Tschofenig, Location ConfigurATION EXTENSIONS FOR PoliCy MANAGEMENT, IETF RFC 7199 (Standards Track), Apr. 2014.

11. J. Peterson, O. Kolkman, H. Tschofenig and B. Aboba, ArChitectural ConsiderATIONS ON APPLICATION FEATURES IN THE DNS, IETF RFC 6950 (Informational), Oct. 2013.

12. J. Winterbottom, H. Tschofenig, H. Schulzrinne, M. Thomson, A Location DeREFERENCE PRotocol Using HTTP-EnAbled Location DELIVERY (HELD), IETF RFC 6753 (Standard Track), Oct. 2012.

13. H. Schulzrinne, H. Tschofenig, Synchronizing Service Boundaries ANd Elements Based on the Location-TO-Service Translation (LoST) Protocol, IETF RFC 6739 (Experimental), Oct. 2012.

14. R. Mahy, B. Rosen, H. Tschofenig, Filtering Location Notifications in the SesSION INITIATION PROTOCOL (SIP), IETF RFC 6447 (Standards Track), Jan. 2012.

15. H. Schulzrinne, L. Liess, H. Tschofenig, B. Stark, and A. Kuett, Location Hiding: Problem Statement and Requirements, IETF RFC 6444 (Informational), Jan. 2012.

16. R. Barnes, M. Lepinski, A. Cooper, J. Morris, H. Tschofenig, H. Schulzrinne, AN ARCHITECTURE FOR LOCATION AND LOCATION PRIVACY IN INTERNET APPLICATIONS, IETF RFC 6280/BCP 160 (Best Current Practice), Jul. 2011.

17. J. Winterbottom, M. Thomson, H. Tschofenig, R. Barnes, UsE of DeviCE IdENTITY IN HTTP-Enabled Location DELIVERy (HELD), IETF RFC 6155 (Standards Track), Mar. 2011. 
18. J. Fischl, H. Tschofenig, E. Rescorla. Framework for Establishing a Secure Real-time Transport Protocol (SRTP) SECurity Context Using DataGRAm Transport LAyer SECURITy (DTLS), IETF RFC 5763 (Standards Track), May 2010.

19. H. Tschofenig, and H. Schulzrinne, GEOPRIV LAYER 7 LOCATION CONFIGURATION Protocol: Problem Statement AND Requirements, IETF RFC 5687 (Informational), Mar. 2010.

20. H. Schulzrinne, V. Singh, H. Tschofenig, M. Thomson, Dynamic Extensions to the PRESENCE INFORMATION DATA Format LOCATION OBJECT (PIDF-LO), IETF RFC 5962 (Standards Track), Sep. 2010.

21. H. Tschofenig, Ed., F. Adrangi, M. Jones, A. Lior, B. Aboba, Carrying Location OBJECTS IN RADIUS AND DiAMETER, IETF RFC 5580 (Standards Track), Aug. 2009.

22. J. Winterbottom, M. Thomson, H. Tschofenig, GEOPRIV PRESEnCE Information Data Format Location ObJeCt (PIDF-LO) USAGE Clarification, CONSIDERATIONS, AND RECOMMENDATIONS, IETF RFC 5491 (Standards Track), Mar. 2009.

23. H. Schulzrinne, J. Polk, H. Tschofenig, Discovering Location-To-Service Translation (LoST) Servers Using the Dynamic Host Configuration PROTOCOL (DHCP), IETF RFC 5223 (Standards Track), Aug. 2008.

24. T. Hardie, A. Newton, H. Schulzrinne, H. Tschofenig, LoST: A Location-TOService Translation Protocol, IETF RFC 5222 (Standards Track), Aug. 2008.

25. T. Taylor, Ed., H. Tschofenig, H. Schulzrinne, M. Shanmugam, SECurity Threats AND REQUiREMENTS FOR EMERGENCY CALl MARKING AND MAPPING, IETF RFC 5069 (Informational), Jan. 2008.

The following additional publications have been issued during my PhD studies:

1. K. Zandberg, K. Schleiser, F. Acosta, H. Tschofenig, and E. Baccelli, Secure Firmware Updates for Constrained IoT Devices using Open Standards: A Reality Check, IEEE Access, May 2019.

2. H. Schulzrinne and H. Tschofenig (Eds.), Internet Protocol-BASEd EMERGEnCY SERVICES, John Wiley \& Sons, Inc., May 2013.

3. EENA ${ }^{4}$, EENA Next Generation 112 - Long Term Definition, Apr. 2012. Available at http://www.eena.org/uploads/gallery/files/pdf/eena_ng112_ longtermdefinition.pdf.

4. H. Tschofenig, Security Risks in NeXt-Generation Emergency Services, in Communications of the ACM, Volume 54 Issue 11, Pages 23-25, Nov. 2011.

\footnotetext{
${ }^{4}$ The EENA Next Generation 112 - Long Term Definition specification was created by the EENA Technical Committee. I am listed on the contributors page of the document.
} 
5. NENA ${ }^{5}$, Detailed Functional and InTERfaCe Standards For the NENA i3 Solution - StAGe 3, NENA 08-003 v1, Jun. 2011. Available at https://www . nena. org/?page=i3_Stage3.

6. H. Tschofenig and H. Schulzrinne, EMERGENCY SERVICES FOR InTERnet MultimeDIA, in ”The Internet Protocol Journal”, Volume 13, No.4, Pages 2-17, Dec. 2010.

\footnotetext{
${ }^{5}$ The Detailed Functional and Interface Standards for the NENA i3 Solution - Stage 3 specification was created by the NENA Long Term Definition Working Group. I am listed on the contributors page of the document.
} 



\section{Chapter 2}

\section{Requirements}

RFC 5012 [5] captures a list of requirements developed by the IETF ECRIT working group and defines the basic terminology for use with IP-based emergency services.

In total, 43 requirements have been identified, and these requirements are categorized into four areas, namely

- 8 high-level requirements,

- 9 requirements related to the caller's location,

- 9 requirements related to the emergency service identifier, and

- 17 requirements focused on the mapping protocol.

RFC 5012 also defines what a mapping protocol is:

The primary purpose of the mapping protocol is to produce a PSAP URI drawn from a preferred set of URI schemes such as SIP or SIPS URIs, based on both location information [6] and a service identifier in order to facilitate the IP end-to-end completion of an emergency call. The (emergency) service identifier describes the emergency service, independent of the user interface mechanism, the signaling protocol that is used to reach the service, or the caller's geographic location.

LoST, which is described in detail in Chapter 5, is such a mapping protocol.

The following requirements, which were copied from RFC 5012, are worth highlighting since they had a substantial impact on the design of the described IP-based emergency services architecture and the LoST protocol. 
International applicability (see $\operatorname{Re} 2$ in [5]): Regional, political, and organizational aspects MUST be considered during the design of protocols and protocol extensions that support IP-based emergency calls. It must be possible for a device or software developed or purchased in one country to place emergency calls in another country.

Distributed administration (see Re3 in [5]): Deployment of IP-based emergency services MUST NOT depend on a single central administrative authority. The design of the mapping protocol must make it possible to deploy and administer emergency calling features on a regional or national basis without requiring coordination with other regions or nations.

Multi-mode communication (see Re4 in [5]): IP-based emergency calls MUST support multiple communication modes, including, for example, audio, video, and text.

Anonymous mapping (see Re8 in [5]): The mapping protocol MUST NOT require the true identity of the target for which the location information is attributed. Ideally, no identity information is provided via the mapping protocol.

Incrementally deployable (see Ma3 in [5]): The mapping protocol MUST be designed to support its incremental deployment.

Anywhere mapping (see Ma5 in [5]): The mapping protocol MUST support the ability to provide mapping information in response to an individual query from any (earthly) location, regardless of where the mapping client is located, either geographically or by network location. The mapping client, such as an Emergency Services Routing Proxy, may not necessarily be anywhere close to the caller or the appropriate PSAP, but must still be able to obtain mapping information.

RFC 5012 did not list requirements for callbacks from PSAPs, emergency calls by unauthenticated and unauthorized devices, calls from vehicles (so-called eCalls or vehicleinitiated emergency calls), and calls triggered by Internet of Things devices. These requirements were added later and can be found in the following publications:

PSAP Callback: The requirements and a problem statement for PSAP callback, which arises from the need for the call taker to reach out to the caller for further information, is described in RFC 7090 [7]. In some jurisdictions, support for offering preferential treatment for PSAP callback is a regulatory requirement.

Emergency Calls from Unauthenticated and Unauthorized Devices: RFC 7406 [8] introduces emergency services support for unauthenticated and unauthorized devices and defines terminology. In some countries, support for such functionality is a regulatory requirement (even though it makes the emergency services architecture more vulnerable to attacks against the emergency services infrastructure and PSAP call taker resources in particular).

Vehicle-initiated Emergency Calls: RFC 8148 [9] outlines the concept of vehicle- 
initiated emergency calling where emergency calls are triggered automatically by vehicles in the event of a crash or serious incident, or manually invoked by a vehicle occupant. In addition to voice and location data, these emergency calls may also convey information generated by sensors.

Data-only Emergency Calls: Data-only emergency calls go one step further and allow Internet of Things devices, such as temperature sensors, burglar alarms, or chemical spill sensors, to communicate generic data to the emergency services infrastructure, as explained in [10].

While the previously referenced documents all come with a description of security threats and requirements, a dedicated security threat and requirements document has been published in RFC 5069 [11]. Many of the attacks concern the use of the mapping protocol so that an attacker is unable to

- inject fake messages,

- mount denial-of-service attacks,

- impersonate the mapping architecture towards other emergency services entities,

- eavesdrop on the communication.

Requirements for communicating location by reference (LbyR), which is a feature offered in addition to the basic location by value (LbyV), were published in RFC 5808 [12]. LbyR offers performance benefits in mobile environments and can also help to improve access control and increase privacy protection. A protocol for requesting and receiving LbyR is needed, however, and the requirements for such a protocol are discussed in a separate publication, namely RFC 5687 [13].

Finally, requirements for hiding location information are outlined in RFC 6444 [14]. Location hiding aims to address cases where Internet Access Providers (IAPs) and Internet Service Providers (ISPs) want to withhold precise location information from the endpoint and from the VSP. There are several reasons for the desire to restrict access to location information, which are described in Section 1.2 of [14], and the privacy benefit is only one of the reasons.

Emergency services is a broad field and not every topic is covered in this thesis. The following topics are outside the scope:

- communication from the PSAP towards the first responders using non-IP-based communication,

- authority-to-citizen emergency calling, which is also known as 'early warning',

- any form of social networks for disaster recovery, 
- communication between first responders,

- the use of legacy infrastructure for emergency services, and

- details about the underlying communication infrastructures (e.g., radio networks). 


\section{Chapter 3}

\section{Related Work}

The work in this thesis focuses on the emergency services architecture described in [15]. However, other solutions have been proposed as well, and in this chapter the most popular approaches are described.

\subsection{NENA i2}

NENA i2 was designed with the intention to allow emergency calls placed from VoIP phones to reach legacy PSAPs. It was developed as a transition architecture towards NENA i3, which is an all-IP-based emergency services architecture. To support VoIP emergency services, additional functionality had to be added to the VoIP service provider and the VoIP Positioning Center (VPC) was introduced as a support infrastructure.

NENA i2 was developed by the National Emergency Number Association (NENA), which is "an organization whose mission it is to foster the technological advancement, availability, and implementation of a universal emergency telephone number system in the United States" [16]. The United States has a unique emergency services infrastructure with more than 6,000 PSAPs. Due to the large number of PSAPs, the impression among the designers of NENA i2 was that it would be simpler to upgrade the VoIP service provider infrastructure rather than the PSAP infrastructure.

Figure 3.1 shows a simplified version of the architecture graphically. The different deployment variants for the VoIP service provider, which allow outsourcing of functionality as well as the use of redirect servers and routing proxies, are not shown. NENA i2 is described in detail in the NENA i2 technical specification $[17,18]$ and summarized in Section 3.1 of [19]. 


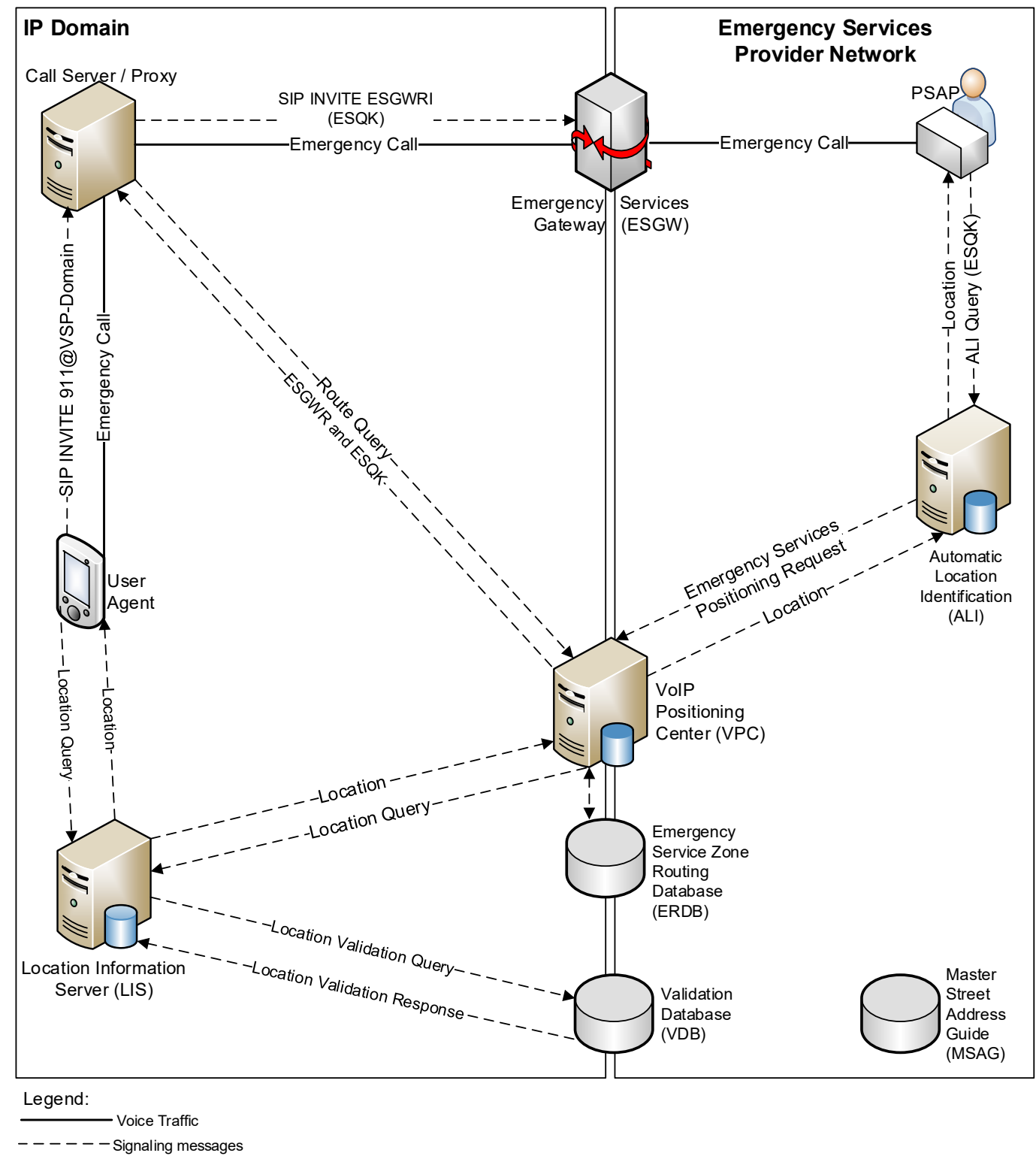

Figure 3.1.: NENA i2 Architecture (Simplified)

Call routing in NENA i2 is intended to allow a VoIP service provider to find a suitable Emergency Services Gateway (ESGW), which then routes the call using the legacy telephony infrastructure to a legacy PSAP. The PSAP needs several information items about a caller, including location, a callback number and subscriber information. Figure 3.1 explicitly highlights the transmission of location information only. Since the legacy infrastructure does not allow location information to be conveyed in-band with the call-signaling mes- 
sages, a separate lookup procedure by the PSAP is necessary. This query is addressed to the so-called Automatic Location Identification (ALI), which itself needs to interact with the Voice Positioning Center (VPC). The lookup key is the Emergency Services Query Key (ESQK), which is one of the most important data structures used in the NENA i2 architecture. The ESQK is a 10-digit number allocated by the VPC for a given emergency call. It serves as a pointer to the location information, the callback number, and other information maintained for a given emergency call. The ESQK is included in the call signaling message towards the PSAP and subsequently used as a parameter in the query to the ALI/VPC. Conceptually, it is similar to the location-by-reference scheme used by a Location Information Server (LIS).

Since NENA i2 architecture focused on fixed and nomadic use of VoIP, which was the primary form of VoIP deployment in 2003 when the work on the architecture was started, civic location information was given special attention. Fixed emergency services in the United States made use of a database called Master Street Address Guide (MSAG), which was populated by the addressing authority of the relevant regions. Before a civic location address could be used with emergency services, either for routing or for dispatch, it had to be 'MSAG validated'. An 'MSAG-validated' address had to be checked against the addresses of the MSAG database to ensure that the address corresponded to an entry in the database. The NENA i2 architecture assigned the location server the task of verifying civic addresses against the MSAG database, which was connected with the Validation Database (VDB). The architecture assumed the possibility of the end device obtaining location information, by value or by reference, from a local LIS. The VoIP service provider would then pass this information on to the VPC, which would either interact with the LIS to resolve the location reference (called Location Key) or use the provided location information directly for route determination.

A final aspect that deserves discussion is the use of route determination so that the VoIP service provider indeed finds the suitable entry point to the legacy infrastructure and to the ESGW. As mentioned previously, the task of route determination was given to the VPC. The VPC takes location information from the emergency caller's device and routing information from the Emergency Service Zone Routing Database (ERDB) as input. The ERDB stores zone information and associates it with specific ESGWs. In response, the VPC returns the Emergency Services Gateway Route Identifier (ESGWRI), which identifies the specific ESGW as well as the route to the PSAP in the legacy network, in the form of a SIP URI. The VPC also allocates the ESQK for use with the respective ESGW and provides it in the response to the VoIP provider as well.

NENA i2 delegates the complex task of route determination to a single entity, the VPC. The VPC essentially becomes a black box, and it is difficult to see how this architecture would scale to the size of the Internet. Even the task of obtaining location information 
about the end device is unspecified since the interaction between the end device and the VoIP service provider is outside the scope of the NENA i2 architecture. Enhancing the existing database lookup scheme (at the ALI and the VPC) to utilize a new identifier (in the form of the ESQK) is, however, a clever hack.

\subsection{ETSI M493}

The standardization work on emergency services within ETSI, the European Telecommunications Standards Institute, was created out of a mandate from the European Commission. This mandate had the number 493 [20] and, as it happens often in Europe, ETSI felt the need to start a standardization activity to fulfill the mandate. In their mandate the European Commission observed that there are VoIP provides that are not 3GPP IMS providers and that those are also required to provide emergency services support to their users. Location information is required so that emergency call routing can take place. Additionally, the ability to transfer location information to the PSAPs for dispatch is needed. The mandate led to the formation of the ETSI M493 group, which first developed an architecture followed by the protocol specifications. The architecture is published in [21] and the protocol specification is available for download at [22].

Figure 3.2 shows a simplified version of the architecture graphically. Details about the emergency services network, which the M493 architecture splits into the Emergency Call Services Provider and the PSAP Service Provider, were omitted. Furthermore, various entities in the emergency services network can query the Location Server, which is simplified in the drawing. Unlike NENA i2, the architecture described in M493 particularly aims to address cases where emergency services support is provided in one country while the Voice Service Provider is operating in a different country. The interface between the user equipment of the emergency caller and the Voice Service Provider is outside the scope of the architecture. Quite naturally, this design decision forces the VoIP service provider to interact with the access network provider and the emergency services infrastructure. Additionally, M493 considers the case of IP-based PSAPs in addition to legacy non-IP-based PSAPs, but focuses primarily on voice-based emergency calling.

The most essential part in the M493 architecture is the Location Server discovery component that uses the source IP address (and source port) of the emergency call as the key for the lookup procedure. RFC 7216 [23] standardizes the discovery steps, which are re-used in the M493 protocol [22]. For cases in which intermediaries such as VPN gateways or TURN servers are used along the media path, the discovery procedure would lead to incorrect results since the information in the network and transport headers is modified in transit. The M493 architecture addresses this challenge and recommends that the discovery procedure 


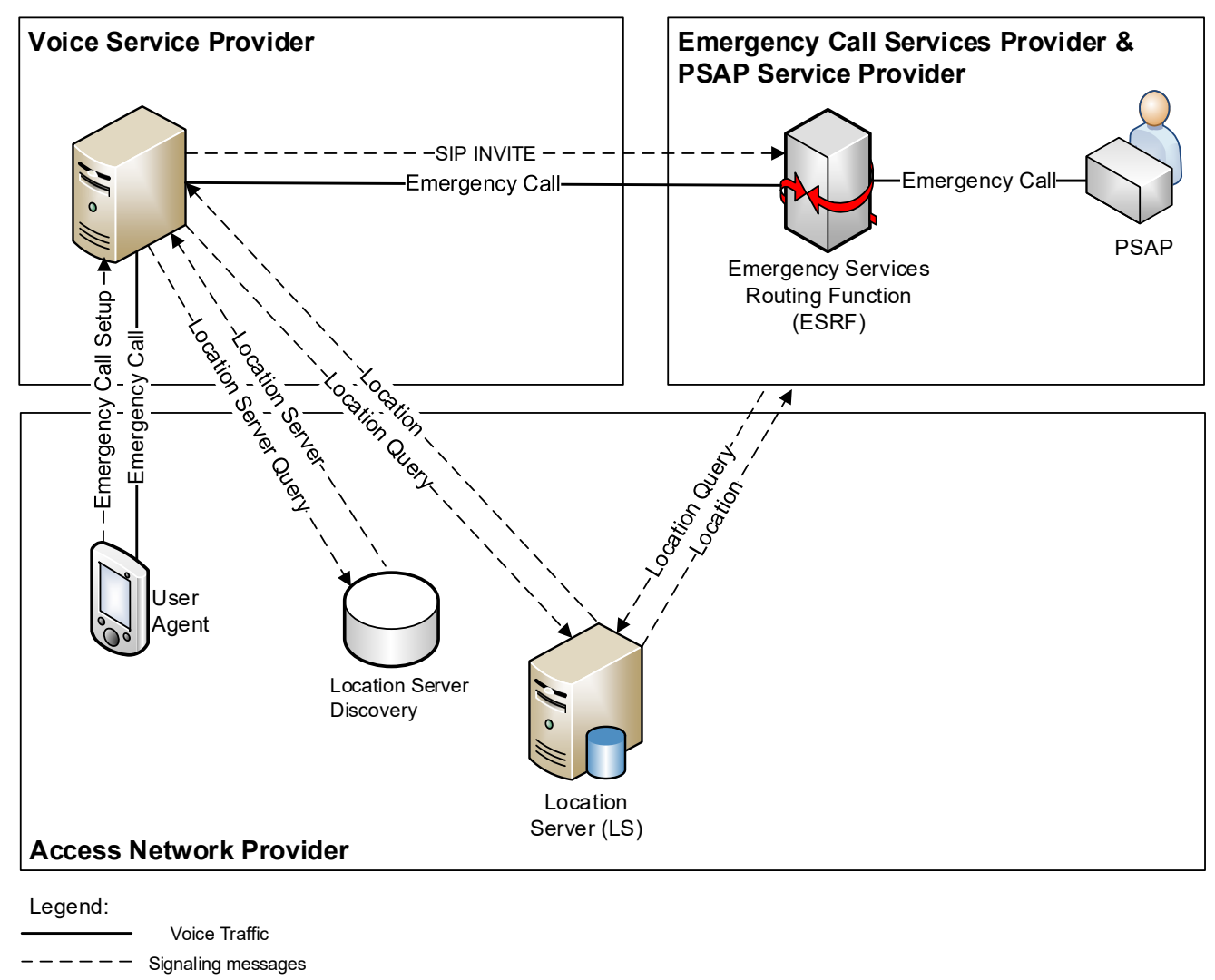

Figure 3.2.: M493 Architecture (Simplified)

be applied in a nested (or chained) fashion. For more details see Appendix A of [21].

Once the Location Server has been discovered the Voice Service Provider uses the HTTPEnabled Location Delivery (HELD) [24-27] protocol or the Diameter protocol [28] to interact with the Location Server. HELD was initially designed to obtain location information, by value or by reference, but was later extended to also return emergency services call routing information [27].

According to the M493 specification, HELD shall be used when no pre-established relationship exists between the Voice Service Provider and the Access Network Provider. When a pre-established relationship exists then the M493 specification allows Diameter, as specified in ETSI ES 283035 [29], to be used as an alternative solution. Civic and geospatial location information is conveyed as defined in IETF RFC 4776 [30], IETF RFC 3825 [31], or as a Presence Information Data Format Location Object (PIDF-LO) defined in IETF RFC 4119 [6]. 


\subsection{DNS-SOS}

With the DNS-SOS proposals [32] Brian Rosen (Neustar) suggested to using a DNS-based mechanism to lookup emergency calling URIs and related emergency information. The proposal assumes a new root, sos.arpa, to be maintained by an international organization that then uses country-level agencies to manage the next-lower level in the hierarchy. For example, at.sos.arpa would be maintained by an Austrian agency, which can then make further delegations for individual states, and cities, if necessary.

DNS-SOS makes use of the Dynamic Delegation Discovery System (DDDS) framework [33], which is a generic mechanism for storing application data in the DNS. This requires a client-side algorithm for transforming a string into a domain name, which is subsequently resolved by the DNS to retrieve Naming Authority Pointer (NAPTR) Resource Record records [33-36]. In this proposal the input is location information, which is then transformed into a so-called Application Unique String. This solution, as RFC 6950 [37] explains, allows the resolution of identifiers, such as telephone numbers or location information, that do not have a recognizable domain component to be treated as domain names for use with the DNS.

A NAPTR record contains the following structures:

- two different weighting mechanisms ("order" and "preference"),

- a "service" field (such as 'sos+police') to express the application the NAPTR record describes,

- a "replacement" field or a "regular expression" field, which performs the translation.

Examples of NAPTR records can be seen in Figure 3.3.

The labels of the Application Unique String follow the civic location structure defined in $[30,38,39]$. This means that the string is expressed as a series of concatenated civic location tokens whereby tokens are separated by a period. For example, the application unique string 'innsbruck.tirol.at.sos.arpa' indicates that the encoded civic address is in the city of "Innsbruck", in the state of "Tirol", and in the country of "Austria". The exact canonicalization algorithm is not provided in the proposal. To take spelling variations into account Brian Rosen including alternative spellings in databases as well. When certain civic tokens from the hierarchy must be omitted, they would be expressed by the '.null' value.

To form an Application Unique String using geodetic location information the proposal suggests to express latitude, longitude, and altitude by concatenating these three values separated with a period. For example, 101d221.93d0345.0.geo.sos.arpa would be a geodetic 
location with 101.221 degrees latitude, 93.0354 degrees longitude, and no altitude information $^{6}$.

In addition to returning service URIs, the DNS-SOS proposal also envisions the following metadata to be provided via NAPTR records:

- a URI to a document containing PSAP URIs and service boundaries (in the form of polygons),

- a URI to a document containing subdomains. The aim of providing this information is for searching, and

- a URI to a document containing building information. This information is meant to be used by first responders rather than for emergency call routing purposes and may require confidentiality protection and access control.

Using the subdomains in combination with the service boundaries would allow a DNS server to respond to queries that include geodetic location information since the server can collect the necessary information to use a point-in-polygon operation to determine which PSAP to route the call to. However, the currently deployed DNS infrastructure does not provide such functionality.

A more complete example of a fictional DNS configuration is shown in Figure 3.3. The example has been taken from Section 6.2 of [41] and modified to illustrate the different service types.

In response to the DNS-SOS proposal and related ideas to enhance the DNS with new functionality, the Internet Architecture Board (IAB) published RFC 6950 [37] to discuss the architectural consequences of using the DNS to implement application features. Engineers like to reuse the DNS infrastructure due to its widespread deployment.

However, the DNS may not be a suitable infrastructure for some applications, as pointed out in RFC 6950. In the context of this proposal, the rules for processing requests of regular DNS queries where a hostname is resolved to an IP address are quite different than the resolution necessary to resolve location information together with a service identification to a PSAP URI. In particular, the use of geodetic location information requires complex location-matching procedures, which the deployed DNS infrastructure does not offer.

\footnotetext{
${ }^{6}$ The example has been taken from Section 10 of [32]. Note that the recommendations in Section 5.2 .1 of RFC 5491 [40] either require the coordinate reference system (CRS) WGS 84 to be used, or to at least indicate a coordinate reference system.
} 


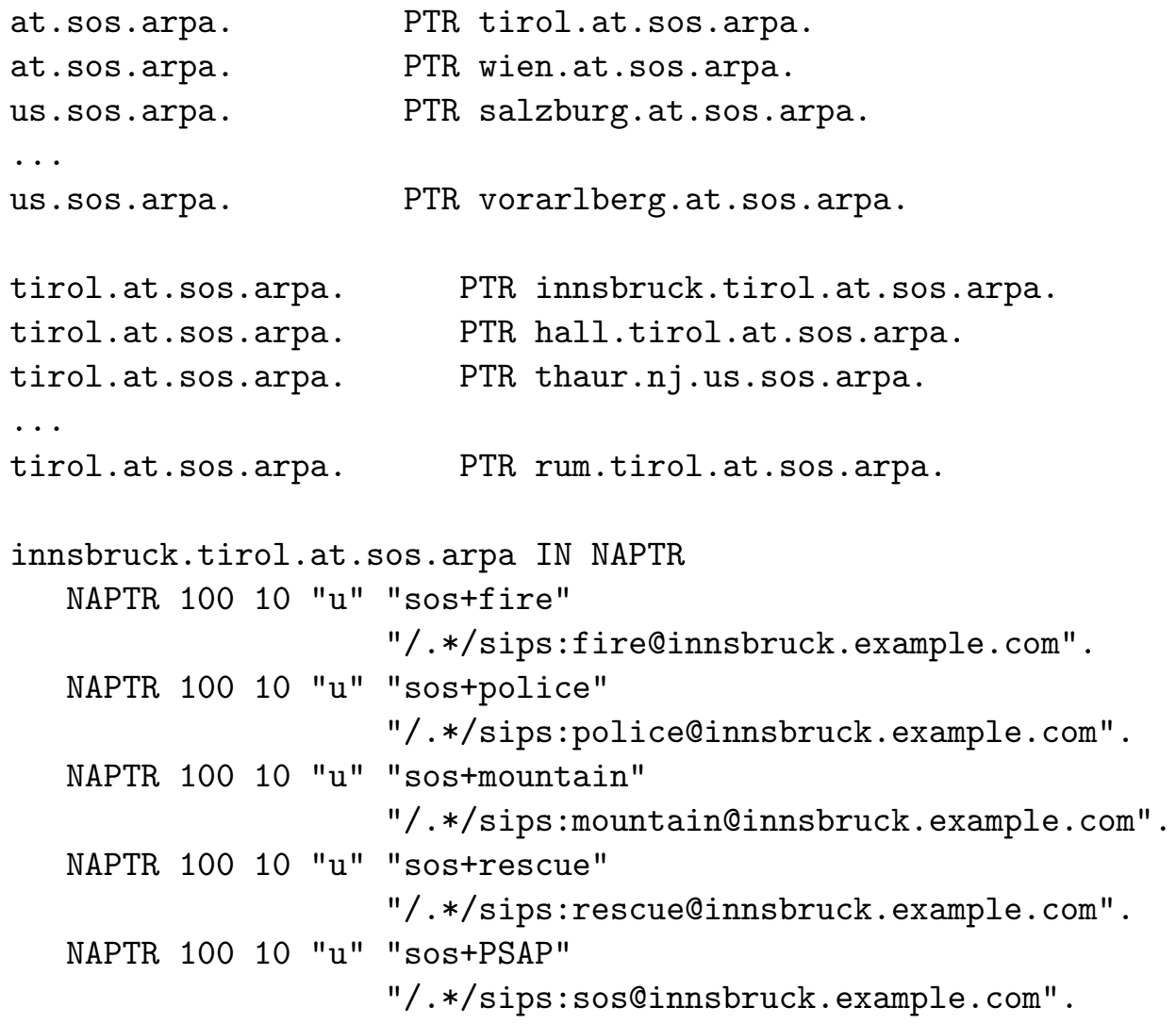

Figure 3.3.: Example DNS-SOS Configuration.

\subsection{GPP IMS}

The IP Multimedia Subsystem (IMS) is an architecture that has been developed by the 3GPP to replace the circuit switched voice infrastructure and has been heavily influenced by its primary customers, i.e., telecommunication operators. The important design decision in the IMS architecture is that the Application Service Provider (ASP) and the Access Network Provider $(\mathrm{ANP})^{7}$ are operated by the same entity. This design decision simplified the lo-

\footnotetext{
${ }^{7}$ The term 'Application Service Provider' (ASP) is defined in RFC 5012 [5] and the term 'Access Network Provider' (ANP) is defined in RFC 5687 [13]. The ANP is a combination of 'Internet Access Provider' (IAP) and 'Internet Service Provider (ISP)', two additional terms defined in RFC 5012 [5]. Note that in some countries there may be a split between the ISP and the IAP, but this separation is not relevant for the description of the IMS emergency services architecture.
} 
cation retrieval and emergency call routing procedure because they happen locally within the visited network. The designers of the IMS architecture also wanted to offer quality of service (QoS) mechanisms for emergency services, as explained in Section 3.6 of [19]. Designing a QoS solution that operates locally is deployable when the application functionality is offered by the network operator.

Gaining access to most networks requires successful completion of the network access authentication procedures. Extra steps had to be added to accommodate cases where the end device ${ }^{8}$ either has no credentials to authenticate to the network or is otherwise not able to perform a successful network access authentication (for example, when there are no roaming agreements or the subscription is not valid).

Figure 3.4 shows a simplified version of the architecture graphically. The main entities in this diagrams are SIP entities, such as the various forms of Call Session Control Functions (CSCFs). CSCFs are SIP servers or proxies. The Emergency Call Session Control Function (E-CSCF) is an entity introduced to provide emergency call handling functionality. It interacts with the Location Retrieval Function (LRF) and communicates with the PSAP in the emergency services network either directly for IP-enabled PSAPs or indirectly via the Border Gateway Control Function (BGCF) and a Media Gateway (MGW) in the case of legacy PSAPs, and focuses on voice- and multi-media signaling exchanges using SIP with the UE detecting the emergency call. The figure does not describe the underlying radio and network attachment procedures. The IMS specifications distinguish the case where the UE is roaming, as shown in Figure 3.4, whereas it is not. In the roaming case, the emergency call is not routed to the home network but instead processed locally via the E-CSCF in what is called a "local breakout". In the roaming case an emergency registration is typically used (unless, for example, it is lacking credentials). The Serving CSCF (S-CSCF), which is located in the home network, is contacted during emergency registration. The InterrogatingCSCF (I-CSCF) is at the edge of the administrative domain and relays communication to the S-CSCF. Non-emergency calls are home routed, and emergency call handling therefore presents an exception in the IMS architecture. Emergency registration uses the "sos" service URN [42] and ensures that a PSAP can initiate a callback, though the PSAP callback itself is not shown in the figure. The Proxy CSCF (P-CSCF), which is the first point of contact for an IMS terminal, must also be provisioned with emergency service URNs and emergency numbers to detect an emergency call from the IMS terminal so that the E-CSCF can be selected and call setups forwarded to it. After the SIP call establishment is complete, voice communication can be initiated, and as shown in Figure 3.4 a gateway may be used to translate the call to the PSTN.

\footnotetext{
${ }^{8}$ The 3GPP uses the terms 'User Equipment' (UE) for a mobile IMS phone with a (U)SIM and 'Mobile Equipment' (ME) for a phone without a (U)SIM and therefore no subscription.
} 


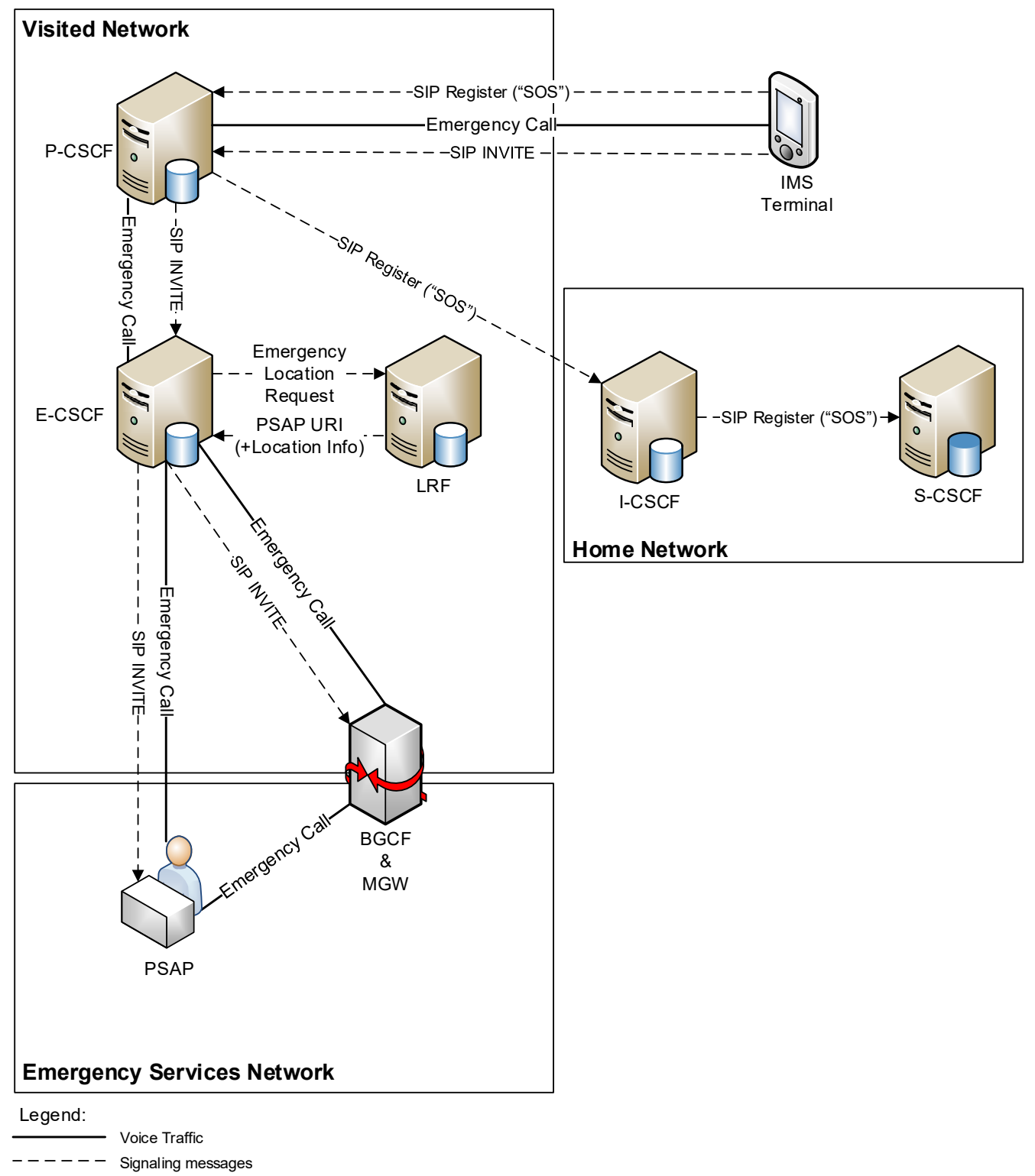

Figure 3.4.: IMS Architecture (Simplified) 


\section{Chapter 4}

\section{Comparison}

The requirements outlined in Chapter 2 lead to the development of the IETF emergency services architecture and the design of the LoST protocol, as described in Chapter 5. In this chapter a comparison between the IETF emergency services architecture and the alternatives i.e. NENA i2, ETSI M493, DNS-SOS, and 3GPP IMS, is provided.

\subsection{NENA i2}

NENA i2 is an early transition solution and lacks many of the features later demanded.

International applicability: NENA i2 was designed specifically for the United States with applicability to Canada. Architecture focused on fixed and nomadic use of VoIP and on the interworking with legacy PSAPs only.

Distributed administration: Route determination is provided by the VoIP Positioning Center (VPC), which interfaces the Emergency Services Zone Routing Data Base (ERDB) and the Location Information Server (LIS).

Multi-mode communication: Focus of the architecture is on voice, particularly regarding the interworking with legacy PSAPs. Hence, there is no support for multi-mode communication.

Anonymous mapping: The VPC uses the caller's location as input to the route determination process. It does not need the caller's identity but the VSP provides customer information to the VPC in order to make it available to the PSAP. Therefore, no anonymous mapping is provided.

Incrementally deployable: The architecture is meant to be deployable in selected countries. It does, however, require the end devices to support the interaction with a LIS to 
obtain location information by value or by reference. Without this location support the described architecture cannot be deployed incrementally even within a single country.

Anywhere mapping: Although not explicitly stated, the VPC is likely to interact only with authorized VSPs. Furthermore, the ERDB is only accessible by the VPC. As such, the VPC/ERDB are not able to provide mapping information in response to an individual query from any location.

PSAP callback: NENA i2 provides PSAP callback support based on the callback number stored by the VSP although without the security properties described in RFC 7090 [7].

Emergency calls from unauthenticated and unauthorized devices: NENA i2 does not support emergency calls from unauthenticated and unauthorized devices.

Vehicle-initiated emergency calling support: NENA i2 does not discuss Vehicleinitiated emergency calling.

Data-Only emergency calls: NENA i2 does not discuss data-only emergency calls.

\subsection{ETSI M493}

ETSI M493 has been developed long after NENA i2 and takes advantage of some of the more advanced IETF standardization work. The architecture also takes deployments into account where the VSP is not offering IMS-based services. The focus on network operators offering both location information and route determination is a strong requirement.

International applicability: ETSI M493 was the result of a mandate by the European Commission and applicability is within the EU member states. It considers legacy PSAPs as well as IP-based PSAPs.

Distributed administration: Access network providers and Emergency Call Service Providers have to closely cooperate in a single country since location and route determination is provided by the access network providers.

Multi-mode communication: Focus of the architecture is on voice, but video and text support is also mentioned.

Anonymous mapping: The LS does not require caller information to perform a mapping. As such, anonymous mappings are provided.

Incrementally deployable: The architecture requires a strong cooperation between VSPs and access network providers since the VSP has to determine the correct Location Server (LS) based on information in the network layer (in most cases the IP address). There is no dependency on the end device in this architecture, at least conceptually.

Anywhere mapping: The LS can only provide mapping information in response to an query from an emergency caller of the access network provider that operates the LS. 
ETSI M493 anticipates that a LS may interact with other LSs, for example in case of VPN scenarios. Whether this concept indeed works in practice is unknown.

PSAP callback: Details about PSAP callback are not described.

Emergency calls from unauthenticated and unauthorized devices: ETSI M493 does not support emergency calls from unauthenticated and unauthorized devices.

Vehicle-initiated emergency calling support: ETSI M493 does not discuss Vehicleinitiated emergency calling.

Data-Only emergency calls: ETSI M493 does not discuss data-only emergency calls.

\subsection{DNS-SOS}

DNS-SOS was an early attempt to untangle location determination from route determination in a way that it can be deployed internationally. Re-using the DNS infrastructure was clever but also revealed a number of problems. Since the proposal was discontinued in favor of LoST only a sub-set of the features are summarized below.

International applicability: DNS-SOS was meant to be used internationally, similarly to the DNS.

Distributed administration: DNS-SOS allows distributed administration. For geodetic location information the proposal was underspecified and prevented the use of the regular DNS infrastructure due to the new search and matching procedure. The single root of the DNS infrastructure may, however, make deployments in certain regions more difficult (particularly when there is a dispute over a geographical region).

Anonymous mapping: DNS-SOS allows anonymous mapping.

Incrementally deployable: DNS-SOS is incrementally deployable and the civic mapping is certainly easier to deploy even though new DNS infrastructure elements are required. At the time when the DNS-SOS proposal was made the DNS offered very few security mechanisms, which may have negatively impacted its deployment (as it was seen with other DNS extensions).

Anywhere mapping: It was envisioned that DNS-SOS allows mapping from anywhere.

\subsection{GPP IMS}

The 3GPP IMS emergency services architecture is an advanced IP-based communication system developed for network operators who are also offering voice over IP services. As 
such, the classical telecommunication operators are the main audience for an IMS-based system.

International applicability: The 3GPP IMS is applicable to IMS-based systems only. It is internationally applicable once network operators in all countries deploy IMS. In the meanwhile those devices will fall back to PSTN-based emergency calling. Non-IMSbased service providers are excluded from emergency services support.

Distributed administration: The assumption of the IMS architecture is that the Application Service Provider (ASP) and the Access Network Provider (ANP) are operated by a single company, or are in close relationship. As such, route and location determination is accomplished by the same entity.

Multi-mode communication: IMS aims to support voice, video and text.

Anonymous mapping: Anonymous mappings are possible when the emergency caller has not been authenticated by the network. In all other cases the involved IMS entities have information about the calling party.

Incrementally deployable: With the legacy emergency call support the 3GPP IMS architecture is incrementally deployable. Support for IMS does, however, require support from the network

Anywhere mapping: In IMS an emergency caller can only expect route determination provided by the IMS entities in the access network. As such, there is no need for a mapping to be provided by a non-local entity.

PSAP callback: Functionality for PSAP callback is provided.

Emergency calls from unauthenticated and unauthorized devices: Emergency calls from unauthenticated and unauthorized devices is supported.

Vehicle-initiated emergency calling support: Vehicle-initiated emergency calls are supported.

Data-Only emergency calls: Data-only emergency calls are supported theoretically but are not described.

Table 4.1 compares NENA i2, ETSI M493, 3GPP IMS and the IETF emergency services architectures against each other. As it can be seen, the IETF emergency services architecture using LoST is the most versatile architecture and various emergency services features developed for the IETF architecture have subsequently been re-used in other architectures as well. 


\begin{tabular}{|l|l|l|l|l|}
\hline Feature & NENA i2 & ETSI M493 & 3GPP IMS & IETF \\
\hline International applicability & $\mathrm{N}$ & $\mathrm{P}$ & $\mathrm{F}$ & $\mathrm{F}$ \\
\hline Distributed administration & $\mathrm{N}$ & $\mathrm{P}$ & $\mathrm{P}$ & $\mathrm{F}$ \\
\hline Multi-mode communication & $\mathrm{N}$ & $\mathrm{P}$ & $\mathrm{F}$ & $\mathrm{F}$ \\
\hline Anonymous mapping & $\mathrm{N}$ & $\mathrm{F}$ & $\mathrm{P}$ & $\mathrm{F}$ \\
\hline Anymous location & $\mathrm{N}$ & $\mathrm{F}$ & $\mathrm{P}$ & $\mathrm{F}$ \\
\hline Incrementally deployable & $\mathrm{N}$ & $\mathrm{P}$ & $\mathrm{F}$ & $\mathrm{F}$ \\
\hline Anywhere mapping & $\mathrm{N}$ & $\mathrm{P}$ & $\mathrm{N}$ & $\mathrm{F}$ \\
\hline PSAP callback & $\mathrm{P}$ & $\mathrm{ND}$ & $\mathrm{F}$ & $\mathrm{F}$ \\
\hline Emergency calls from unauthenticated and unauthorized devices & $\mathrm{ND}$ & $\mathrm{ND}$ & $\mathrm{F}$ & $\mathrm{F}$ \\
\hline Vehicle-initiated emergency calling support & $\mathrm{ND}$ & $\mathrm{ND}$ & $\mathrm{F}$ & $\mathrm{F}$ \\
\hline Data-Only emergency calls & $\mathrm{ND}$ & $\mathrm{ND}$ & $\mathrm{ND}$ & $\mathrm{F}$ \\
\hline Signaling security & $\mathrm{ND}$ & $\mathrm{ND}$ & $\mathrm{F}$ & $\mathrm{F}$ \\
\hline Media security & $\mathrm{ND}$ & $\mathrm{ND}$ & $\mathrm{F}$ & $\mathrm{F}$ \\
\hline Support for non-IMS end devices & $\mathrm{F}$ & $\mathrm{F}$ & $\mathrm{N}$ & $\mathrm{F}$ \\
\hline Support for non-SIP end devices & $\mathrm{F}$ & $\mathrm{F}$ & $\mathrm{F}$ & $\mathrm{F}$ \\
\hline
\end{tabular}

Table 4.1.: Emergency Services Architecture Solution Comparison.

Fully supported (F)

Legend: Partially supported (P)

Not supported (N)

Not described (ND) 



\section{Chapter 5}

\section{An IP-based Emergency Services Architecture}

Figure 5.1 shows the vision of an IP-enabled emergency services architecture graphically. The left side of the picture shows end devices attached to different access networks and communication service providers. In the Internet many applications are provided by companies independent from the access network operator and therefore the separation between the access network and the communication service provider is indicated explicitly whereas in the circuit switched telephony world the application functionality was provided by the access network operator. The right side of Figure 5.1 depicts the IP-based emergency services network. The developed technical specifications focus on both sides and use the Session Initiation Protocol (SIP). While other protocols have been developed in the meanwhile, such as XMPP, RTCWeb, and many proprietary protocols, they have never received the same attention from the emergency services community. Consequently, emergency services standards are primarily available for SIP-based deployments.

In addition to phones, which have typically been used to place emergency calls, other devices have been used to communicate information relevant for emergency calls, including Internet of Things devices that offer sensor data (such as smoke detectors), audio and video information. The ability of placing emergency calls by vehicles has also extended the ability to summon help faster and to provide more detailed information about the incident to the call taker and first responders.

By design, these non-SIP-based communication architectures can, however, also be supported and Figure 5.1 just refers to them as originating networks. Three core features must be provided by these originating networks in order to interwork smoothly with the standardized IP-based emergency services infrastructure: 


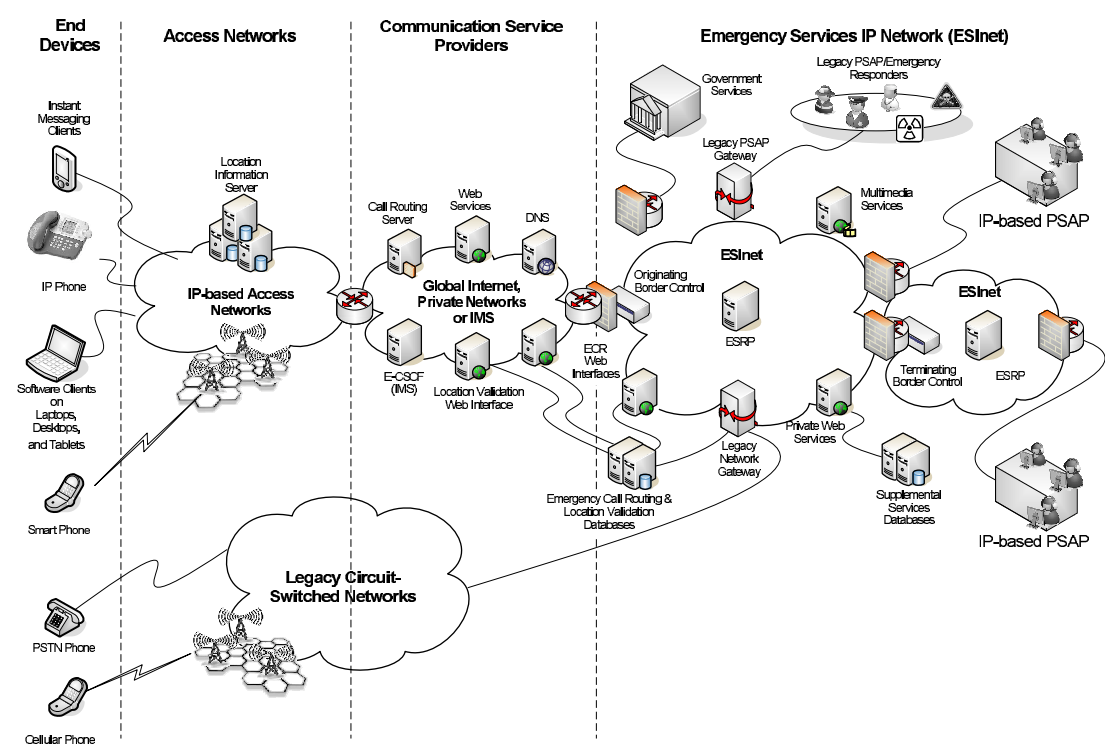

Figure 5.1.: Communication Architecture Overview ${ }^{a}$.

${ }^{a}$ Courtesy of Guy Caron, ENP

1. Ability to identify an emergency call.

2. Ability to communicate location and/or a location reference.

3. Ability to convey multi-media content.

These three core building blocks are described in more detail in Section 5.1. Some of the communication architectures in use today provide support for this functionality, such as the 3GPP IMS architecture ${ }^{9}$, and the IETF SIP architecture. At the time of writing, there are other communication architectures that do not yet support emergency services, such as the Real-Time Communication in WEB-browsers [43] and the Extensible Messaging and Presence Protocol (XMPP) [44]. There are also many non-standardized and proprietary communication architectures, such as Skype ${ }^{\circledR}$, and many smart phone messaging apps (such as WhatsApp).

Although it seems counterproductive to have many different communication architectures the reason for their existence is manifold. The history and background of the persons designing the different communication architectures often had a huge influence on the outcome. In many cases business models dictate design decisions of the work in different standards developing organizations. The following paragraph describes one such design

\footnotetext{
${ }^{9}$ The 3GPP IMS architecture uses the SIP protocol family as a foundation and is a variant of the more generic IETF SIP architecture.
} 
differentiator.

The Internet architecture follows a layered design, which is a common design pattern for communication systems in general, and allows the replacement of different components (such as one radio technology with another one) while only impacting the neighboring layers. In student textbooks, the Internet architecture has the data link layer, network layer, transport layer, and the applications layer. In the real world, the layering is much more complex, and it is sometimes hard to assign specific protocols to specific layers since protocols can be used in a very flexible way and are often tunneled inside other protocols. The responsibility of providing implementations and deployments of certain layers is also distributed to different parties. In many deployments, the provider of physical connectivity (e.g., a wire or radio base stations) and the provider offering Internet connectivity are different companies. Furthermore, those companies offering Internet connectivity are very often different from those offering application layer services. This separation of functionality is a consequence of the end-to-end principle rather than the layering alone. The end-to-end principle states that end-to-end functions can best be realized by end-to-end protocols [45]. While the end-to-end principle still leaves room for discussion and interpretation, many application deployments today happen independently of Internet connectivity deployments. Those who provide Internet connectivity would like to provide applications as well, or at least benefit from the deployment of the applications. Consequently, a design that assumes that the Internet access provider also offers application services is different from a design that assumes a separation between the two parties. Such fundamental design assumptions lead to different communication architectures and consequently also to different designs for the emergency services system even though it is possible to re-use the same building blocks.

The differences between XMPP-based, Skype ${ }^{\circledR}$-based, over-the-top SIP-based VoIP deployments, and RTCWeb are, on the other hand, less dramatic. All three assume independence of the application service provider from the Internet access provider. The differences are mainly in the protocol choice. SIP is an IETF standard and is widely used for voice traffic. Skype ${ }^{\circledR}$ software, on the other hand, uses a proprietary protocol that only relies on SIP for interconnecting with other non-Skype ${ }^{\circledR}$-based systems. XMPP is also an IETF protocol that has found widespread usage for instant messaging. RTCWeb is the most recent standardization development that has been done by the IETF together with the W3C and re-uses Web development programming paradigms that rely on JavaScript and JavaScript APIs. For a discussion about the power of downloadable code, as provided by the Web architecture, read [46].

The term smart phone app just refers to an implementation of some protocol. It does not indicate whether a standardized or a proprietary protocol has been implemented. As such, a smart phone app may be an implementation of any of the protocols listed above, such as a SIP-based VoIP client. In many cases, however, smart phone apps contain proprietary 
communication protocol implementations.

\subsection{Building Blocks}

\section{Recognizing Emergency Calls}

In the early days of PSTN-based emergency calling, callers would dial a local number for the fire or police department. It was recognized in the 1960s that trying to find this number in an emergency caused unacceptable delays. Thus, most countries have been introducing single nationwide emergency numbers, such as 9-1-1 in North America and 1-1-2 in all countries of the European Union. This became even more important as mobile devices started to replace landline phones. As it can be seen from the introduction of 1-1-2 in Europe this education effort takes many years and the old emergency numbers are therefore still in use today (in addition the European-wide 1-1-2 number).

In many countries, different types of emergency services, such as police or mountain rescue, are still identified by separate numbers. Unfortunately, there are more than 60 different emergency numbers in use worldwide, many of which also have non-emergency uses in other countries, so that simply storing the list of numbers in all devices is not feasible. Furthermore, hotels, university campuses, and larger enterprises often use dial prefixes, so that an emergency caller has to dial 0-1-1-2 to reach the fire department when making a call from a landline phone on premise.

With the introduction of smart phones new user interface designs emerged as well. Some devices may use dedicated emergency calling buttons or similar user interface elements to initiate an emergency call. Such mechanisms need to be carefully designed so that they are not accidentally triggered, e.g., when the device is in a pocket.

Instead of conveying the actual dial string in a protocol message once the user has entered it, a symbolic representation is used instead. This allows unambiguous emergency call identification and automatic treatment of calls by VoIP call handling software. The mechanism used for this emergency call marking uses the Uniform Resource Names (URNs) defined in RFC 5031 [42], such as urn:service.sos.

\section{Obtaining and Conveying Location Information}

Location information is needed by emergency services for three reasons: routing the call to a PSAP that is closest to the emergency caller, dispatching first responders (e.g., policemen or ambulance) to the location of the emergency, and determining the emergency 
service dial strings that are supported in a specific area. It is obvious that location has to be determined automatically for the first and third purpose. Experience has shown that automated, highly accurate location information is vital to dispatching as well, rather than relying on the caller to report his or her location to the call taker. This increases accuracy and avoids dispatch delays when the caller is unable to provide location information due to language barriers, lack of familiarity with his or her surroundings, stress, physical or mental impairment. For this reason, automatic location determination and location conveyance for emergency calls are important requirements in nearly all countries.

Location information for emergency purposes comes in two representations: (a) geospatial (or also called geodetic), e.g., longitude and latitude, and (b) civic, e.g., street addresses similar to postal addresses. For indoor location vertical information (floor level) is very useful. Civic locations are utilized for fixed Internet access, including wireless hot spots, and are often preferable for specifying indoor locations in complex buildings, while geodetic location is frequently used for mobile devices, such as cell phones. However, with the introduction of femto and pico cells civic location is feasible with mobile phones and accurate geodetic information can be very hard to acquire indoors.

The requirements for location accuracy differ between routing and dispatch. For call routing, city or even county-level accuracy is often enough, depending on how large the PSAP service areas are, while first responders benefit greatly when they can pinpoint the caller to a building or, better yet, apartment or office for indoor locations, and an outdoor area of at most a few hundred meters outdoors. This avoids having to search multiple buildings, e.g., for medical emergencies.

Various protocol mechanisms have been developed to obtain location information from Location Servers. Devices without GPS may interact with these Location Servers and in certain cases even devices with GPS receivers may contact Location Servers to obtain verified location information or for obtaining GPS-based location information faster using serverprovided assistence data. Chapter 5 discusses various location protocols that have been developed to allow the calling device, the PSAP, or some other proxy on behalf of them to request location information.

\section{Routing Emergency Calls}

Once an emergency call is recognized, the call needs to be routed to the appropriate PSAP. Each PSAP is only responsible for a limited geographic region, its service region. In addition to the geographical region different PSAPs often only provide their service for specific services, such as police, fire, ambulance, etc. There is a wide range of different deployment models used throughout the world and a description of the different PSAP models for Europe can be found in $[47,48]$. 
The number of PSAPs serving a country varies quite a bit. Sweden, for example, has 18 PSAPs and the United States approximately 6,200 (at the time of writing). Therefore, there is roughly one PSAP per 500.000 inhabitants in Sweden and one per 50.000 in the US. As all-IP infrastructure is rolled out, smaller PSAPs may be consolidated into regional PSAPs.

Emergency calls are primarily routed based on the emergency caller's location information. Routing may also take place in multiple stages taking factors, such as the number of available call takers, the load situation of a PSAP, supported media (such as voice, real-time text), or the language capabilities of the call takers into account. It is expected that the importance of dynamic call routing will increase in the near future. In order to perform the initial location-based routing step to get the call setup messages to an entity closer to the PSAP, information about the service boundaries of PSAPs need to be known to end devices, to VSPs or to other call routing entities. This information must somehow be shared. Today, Excel sheets are used [49] to exchange phone numbers associated with PSAP service boundaries. A call taker then must analyze the situation, lookup the appropriate phone number, and manually re-route the call. This may be a reasonable initial step for the small number of transnational emergency calls that are misrouted in today's emergency services system but is not a future-proof approach for a next generation IP-based emergency services system. The inability of emergency centers to quickly re-route calls due to an overload situation in a mass incident has already been recognized [50].

\subsection{Routing Emergency Calls}

In limited scenarios, where the VSP and the ISP role are offered by the same provider routing of IP-based emergency calls can be based on statically pre-configured rules. However, the separation of these two roles to separate companies introduces challenges for emergency call routing. In addition, emergency services authorities are interested to use IP and SIP-based functionality to route calls more dynamically. To decouple the routing enforcement point from the decision point an automated procedure has been standardized. Two mechanisms are available: the service boundary exchange mechanism [51] and the Location to Service Translation (LoST) protocol [52]. LoST may make use of LoST Sync for distributing mappings between trusted distribution points for efficiency purposes. LoST, as illustrated in Figure 5.2, is an HTTP-based query/response protocol where a client sends a request containing the location information and service URN to a server and receives a response, containing the service URL, typically a SIP URL, the service region where the same information would be returned and an indication of how long the information is valid. Both request and response are formatted as XML. For efficiency, responses are cached. The response may also indicate that only a more generic emergency service is offered for this region. For example, a request for 'urn:service:sos.marine' in Austria may be replaced by 


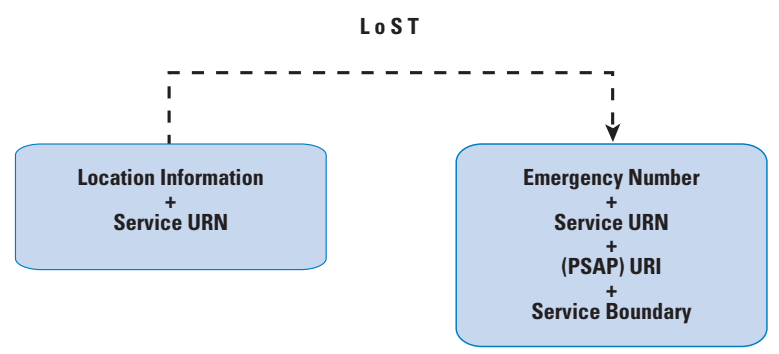

Figure 5.2.: High-Level Functionality of Location-to-Service Translation (LoST).

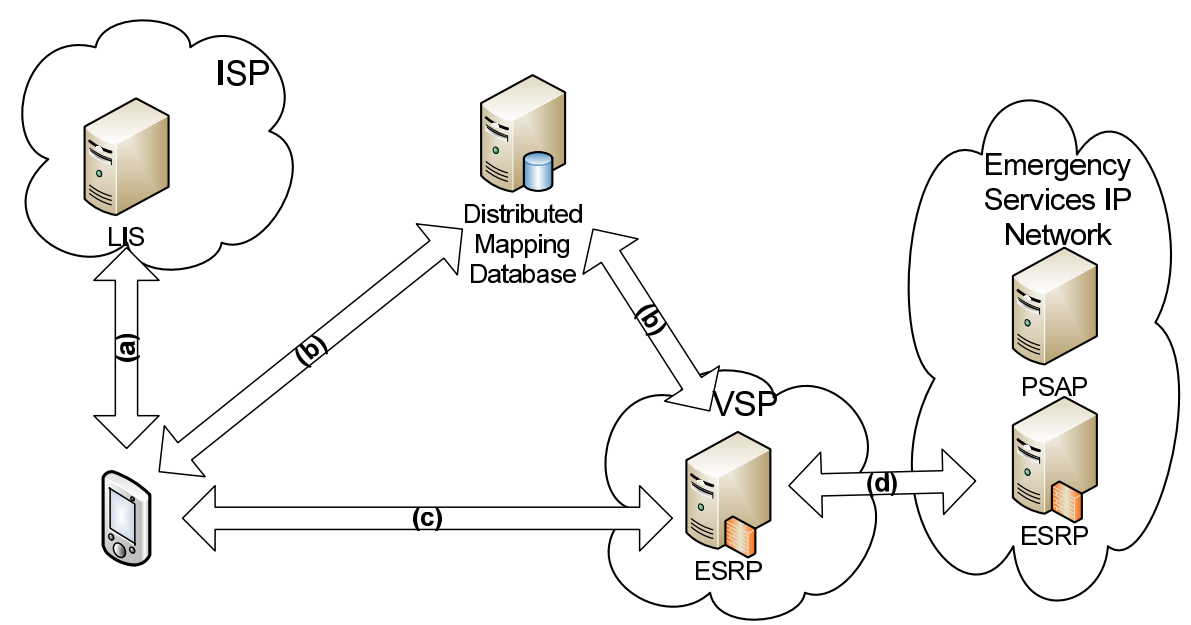

Figure 5.3.: Main Components involved in an Emergency Call.

'urn:service:sos'. Finally, the response also indicates the emergency number / dial string for the respective service.

\subsection{Obligations}

In this section we discuss the requirements the different entities need to satisfy, based on Figure 5.3. A more detailed description can be found in [15]. Note that this narration focuses on the final stage of deployment and does not discuss the transition architecture, in which some implementation responsibilities can be re-arranged, with an impact on the overall functionality offered by the emergency services architecture. A few variations were introduced to handle the transition from the current system to a fully developed ECRIT architecture. 
With the work on the IETF emergency architecture we have tried to balance the responsibilities among the participants, as described below.

\subsubsection{End Hosts}

An end host, through its VoIP application, has three main responsibilities: it has to attempt to obtain its own location, determine the URI of the appropriate PSAP for that location, and recognize when the user places an emergency call by examining the dial string. The end host operating system may assist in determining the device location.

The protocol interaction for location configuration is indicated as interface (a) in Figure 5.3 and several location configuration protocols have been developed to provide this capability. Note that end hosts may also obtain location information via GPS.

A VoIP application needs to support the Location-to-Service Translation (LoST) protocol [52] in order to determine the emergency service dial strings and the PSAP URI. Additionally, the service URNs, defined in [42], need to be understood by the device.

As currently defined, it is assumed that PSAPs can be reached by SIP, but may support other signaling protocols, either directly or through a protocol translation gateway. A LoST response indicates whether other signaling protocols are supported. To provide support for multi-media communication different types of codecs may need to be supported or dynamically negotiated, whereby the details can be found in [15].

\subsubsection{ISP}

The ISP has to make location information available to the end point via one or more of the location configuration protocols.

In order to route an emergency call correctly to a PSAP, an ISP may initially disclose the approximate location for routing to the end point and more precise location information later, when emergency personnel is dispatched by the PSAP operator. The functionality required by the IETF emergency services architecture is restricted to the disclosure of a relatively small amount of location information, as discussed in [14].

The ISP may also operate a caching LoST server to improve the robustness and the reliability of the architecture. This lowers the roundtrip time for contacting a LoST server, and 
the caches are most likely to hold the mappings of the area where the emergency caller is currently located.

In the case where ISPs allow Internet traffic to traverse their network, the signaling and media protocols used for emergency calls function without problems. Today, there are no legal requirements to offer prioritization of emergency calls over IP-based networks. While the standardization community has developed a range of Quality of Service signaling protocols, their widespread deployment has not happened yet.

\subsubsection{VSP}

SIP does not mandate that call setup requests traverse SIP proxies, i.e., SIP messages can be sent directly to the user agent. Thus, even for emergency services, it is possible to use SIP without the involvement of a VSP. However, in terms of deployment, it is highly likely that a VSP will be used. If a caller uses a VSP, this VSP often forces all calls, emergency and regular calls, to traverse an outbound proxy or a session border controller (SBC) operated by the VSP. If some end devices are unable to perform a LoST lookup, the VSP can provide the necessary functions as a back-up solution.

If the VSP uses a signaling or media protocol that is not supported by the PSAP, it needs to translate signaling messages and potentially also media flows.

VSPs can assist the PSAP by providing identity assurance for emergency calls, e.g., using SIP Identity (RFC 3325 [53]), thus helping to prosecute prank callers. However, the link between the subscriber information and the real-world person making the call is often weak. In many cases, VSPs have, at best, only the credit card data for their customers and some of these customers may use gift cards or other anonymous means of payment.

\subsubsection{PSAP}

The emergency services best current practice specification [15] only discusses the standardization of the interfaces from the VSP and ISP towards PSAPs and some parts of the PSAP-to-PSAP call transfer mechanisms that are necessary for emergency calls to be processed by the PSAP. Many aspects related to the internal communication within a PSAP, between PSAPs as well as between a PSAP and first responders are beyond the scope of the IETF specification.

When emergency calling has been fully converted to Internet protocols, PSAPs must ac- 
cept calls from any VSP, as shown in interface (d) of Figure 5.2. Since calls may come from all sources, PSAPs must deploy mechanisms to reduce the number of malicious calls, particularly calls containing intentionally false location information. Assuring the reliability of location information remains challenging, particularly as more and more devices are equipped with Global Navigation Satellite Systems (GNSS) receivers, such as GPS, allowing them to determine their own location [54]. However, it may be possible in some cases to verify the location information provided by an end host by comparing it against network-provided location information.

\subsection{LoST Mapping Architecture}

So far, we have described the LoST protocol as it is described in RFC 5222 [52], namely as a client-server protocol. A single LoST server, however, does not store the mapping elements for all PSAPs worldwide, for both technical and administrative reasons. Thus, there is a need to let LoST servers interact with other LoST servers, each covering a specific geographical region. The LoST protocol already provides the baseline mechanisms for supporting such a communication architecture, as described in RFC 5582 [55], an informational RFC providing terminology (in the form of different roles for LoST servers that distinguish their behavior) and explaining the basic concept of the LoST mapping architecture. RFC 5582 motivates the basic design decision for LoST to utilize it in a wide variety of architectures, but leaves the detailed instantiation to deployments in different jurisdictions.

The awareness of peering LoST servers determines the structure of the architecture rather than certain physical properties of a network, such as the topology of a fiber installation, or the structure of a national emergency services organization. Two types of structures are used in combination, namely a mesh and a hierarchical structure. The mesh topology is envisioned for the top-level LoST entities, whereas the hierarchical structure reflects a parent - child relationship in a tree. Figure 5.4 shows this structure graphically with the LoST servers acting in their roles of forest guides (FGs) and trees. A tree consists of a self-contained hierarchy of authoritative mapping servers (AMS) for a service. An AMS is a LoST server that can provide the authoritative answer to a set of queries. The top-most server in a tree is called the tree root and this server peers with one or multiple FGs, i.e., the tree root announces its coverage region to other FGs. In Figure 5.4, for example, the root of tree 1 interacts with FG $\mathrm{A}$ and makes the coverage area available. FG A also receives the coverage area from the root of tree 2 . All tree roots receive information about the coverage area of their children in the tree. On the top level, all FGs (namely FG A, FG B, and FG C) form a mesh and synchronize their coverage areas.

Seekers and resolvers are two additional LoST entities in the LoST mapping architecture 


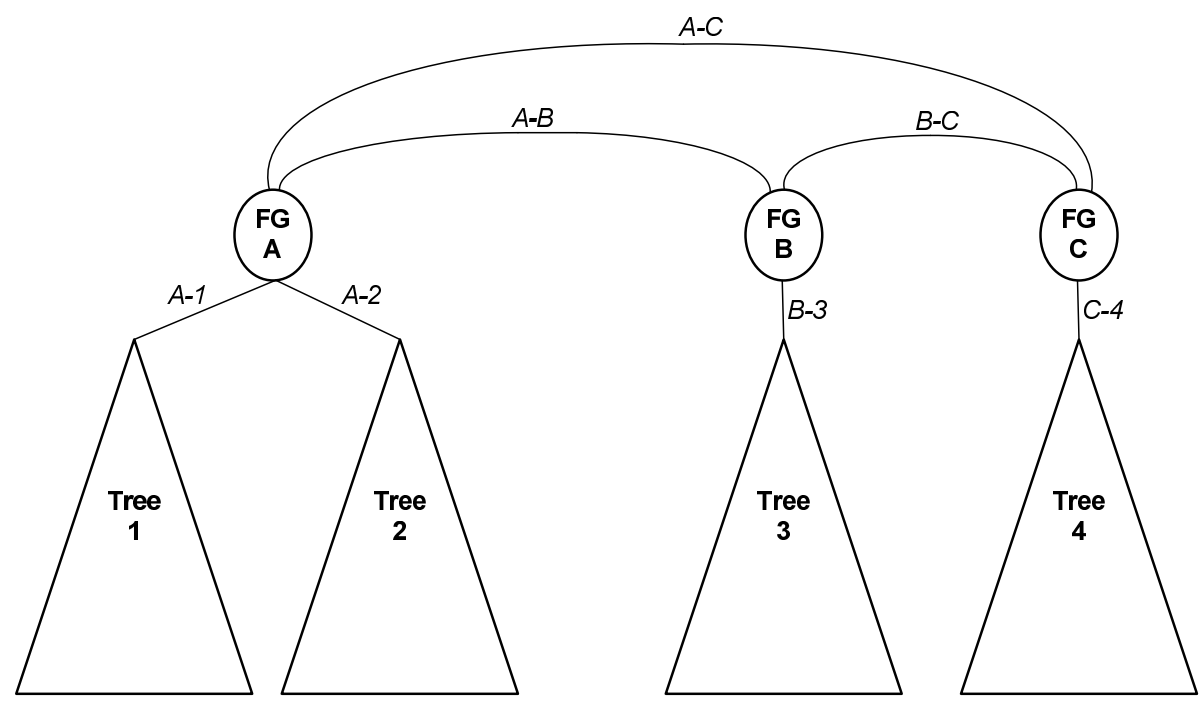

Figure 5.4.: Trees and Forest Guides in the LoST Mapping Architecture.

that are not shown in Figure 5.4. Neither seekers nor resolvers provide authoritative answers themselves but they may cache results. Caching of mapping elements by resolvers is expected to be very common.

To best understand the LoST mapping architecture it is important to highlight the main design goals:

Robustness: To ensure the stability of the system it still needs to function even if different deployment decisions in various parts of the world are made. It cannot be assumed that everyone deploying LoST servers has to agree with everyone else. The minimum level of agreement that has to be ensured is that AMSs are able to authoritatively answer mapping queries, i.e., only those LoST servers respond authoritatively if they indeed have the authority of a specific coverage area.

Consistent Responses: Any device (called seeker) can issue a LoST query and it will get a consistent answer regardless of where the query enters the system. In some (rare) cases of territorial disputes, two AMSs may claim authoritative for the same region. In such a case, the answer received by a seeker will vary depending on the entry point into the mapping system.

Scalability: Scalability of the LoST architecture is ensured by using caching and the distributed nature of the LoST servers in the architecture. Any LoST entity may support caching of received mapping elements. The mapping elements may be obtained as part of the ordinary operation of LoST (via query and responses) but also via separate replication of the mapping elements. LoST Sync [51] is one such protocol to exchange mappings 
between LoST servers (and other entities).

Minimal Seeker Configuration: A seeker is a LoST client requesting a mapping. The only information a seeker needs to know is the address of a resolver; it does not need to know the structure of all Forest Guides nor does it need to maintain a global picture of LoST servers. To avoid having end user involvement in the configuration of LoST servers, Section 4 of the LoST specification [52] provides a discovery technique based on DNS, and RFC 5223 [56] offers a DHCP-based discovery procedure. Although LoST servers can be located anywhere, a placement topologically closer to the end host, e.g., in the access network, may be desirable in disaster situations with intermittent network connectivity. RFC 5223 offers this capability.

Even though it is technically possible to let seekers and resolvers enter their queries at any point in the LoST mapping architecture, a deployment choice is to configure resolvers with the addresses of the FGs. A query and response for an emergency caller located in Germany with a service provider in Finland could then be shown as depicted in Figure 5.5. In our example we assume that the VSP deploys a LoST resolver that is contacted by LoST seekers. We furthermore assume in this example that no caching takes place to illustrate the message flow (as shown with dotted lines). In message (1) the seeker contacts its pre-configured resolver with a recursive query providing its current location (somewhere in Germany). The resolver does not have any information about the PSAP that must be contacted for the given location in Germany (for the solicited service). Since the resolver knows the address of the FG (only one FG is shown in our example), it issues an iterative query to it, as marked with message (2). The FG responds with the entry point for the German LoST tree. The resolver then issues another query towards the provided tree root in message (3). In this example, we assume that the root of tree 1 knows the address of the PSAP the seeker must contact. This final response is then forwarded to seeker via the resolver. The resolver would want to cache the intermediate and final results to speed-up later lookups for the same geographical area and the same service. Once the seeker knows the final answer, it can proceed with the emergency call setup procedure to contact the PSAP, as shown in message (4) with the double line. (Note that the response for a different service may yield a somewhat different service boundary.)

As illustrated, LoST servers form a distributed mapping database, with each server carrying mapping elements. These mapping elements are the main data structure that is communicated in the LoST protocol, synchronized between FGs and LoST servers in the tree, and cached by resolvers and seekers. Figure 5.6 shows the data elements of this important data structure graphically. 


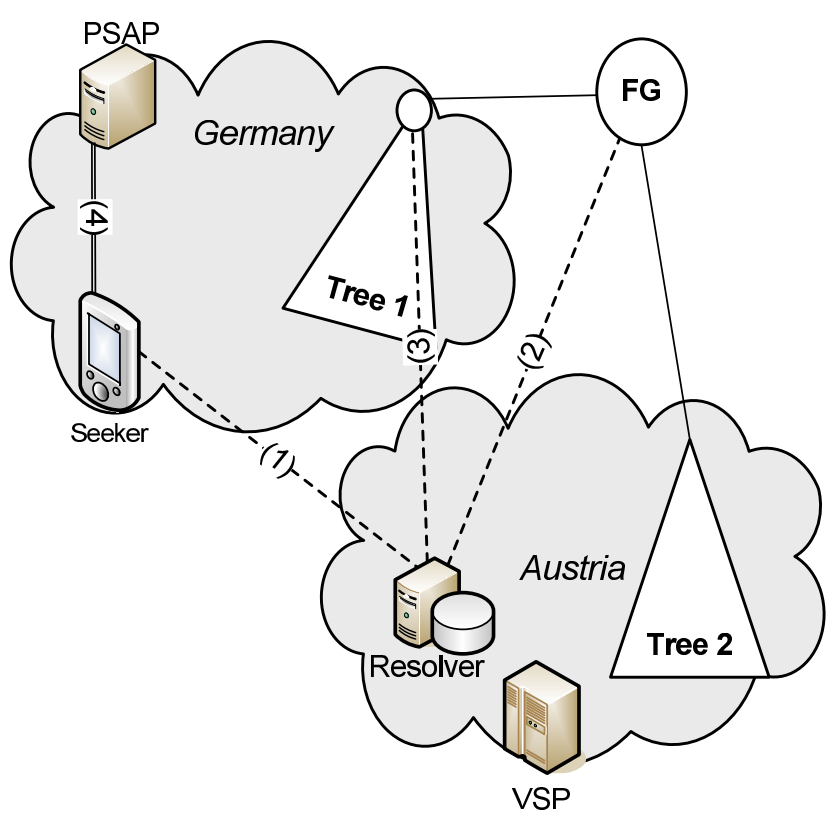

Figure 5.5.: Example Query / Response in the LoST Mapping Architecture.

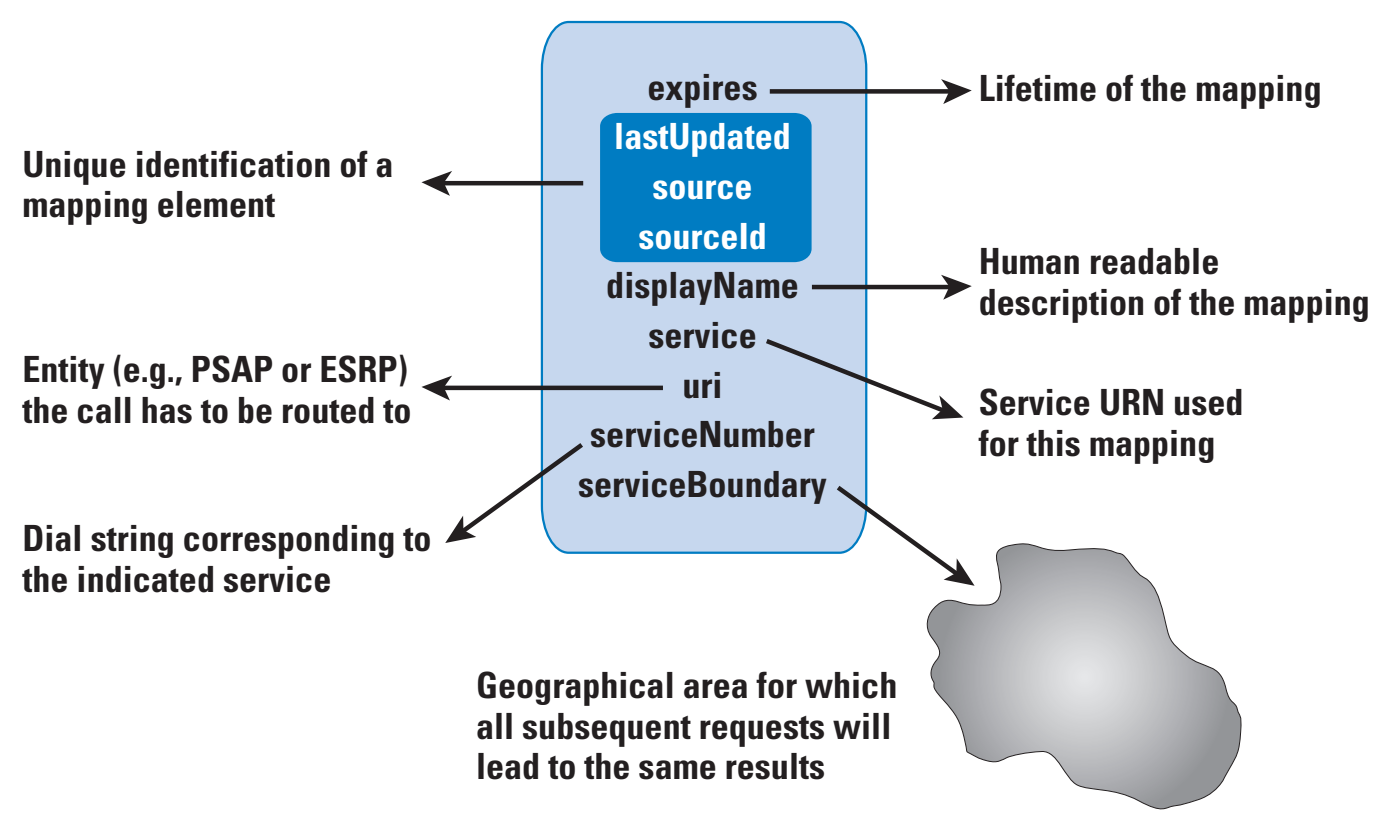

Figure 5.6.: Mapping Element. 


\subsection{Steps towards an IETF Emergency Services Architecture}

The architecture described so far requires changes both in already-deployed VoIP end systems and in the existing PSAPs. The speed of transition and the path taken varies between different countries depending on funding and business incentives. As such, it is difficult to argue whether upgrading end points will be easier or replacing the emergency service infrastructure. In any case, the transition approaches being investigated consider both directions. We can distinguish roughly four stages of transition:

- In the first stage VoIP end systems cannot place emergency calls at all.

- In a second stage, VoIP callers manually configure their location, and emergency calls are routed to the appropriate PSAP as circuit-switched calls via VoIP-PSTN gateways. This level of service is offered in some countries for PSTN-replacement VoIP services, i.e., VoIP services that are offered as replacement for the home phone. In the United States, this is known as the "NENA i2" transition architecture.

- In a third stage, PSAPs maintain two separate infrastructures, one for calls arriving via an IP network, and another one for calls originating in the legacy infrastructure.

- In the final stage, all calls, including those from traditional cell phones and analog landline phones, reach the PSAP via IP networks, with the legacy calls converted to IP-based calls, based on the requirements outlined in [57], either by the carriers or by gateways at the edge of the emergency service infrastructure.

\subsubsection{Legacy End Points}

Figure 5.6 shows an emergency services architecture with legacy end points. When the emergency caller dials the European-wide emergency number '112' (step 0), the device treats it as any other call without recognizing it as an emergency call, i.e., the dial string provided by the end point that may conform to RFC 4967 [58] or RFC 3966 [59] is signaled to the VSP (step 1). Recognition of the dial string is then left to the VSP for processing/sorting; the same is true for location retrieval (step 2) and routing to the nearest (or appropriate) PSAP (step 3). Dial string recognition, location determination and call routing are simpler on a fixed device and voice / application service provided only by the ISP than when they are provided via separate a VSP and ISP.

If devices are used in environments without location services, the VSP's SIP proxy may need to insert location information based on estimates or subscriber data. We briefly describe these cases below. 


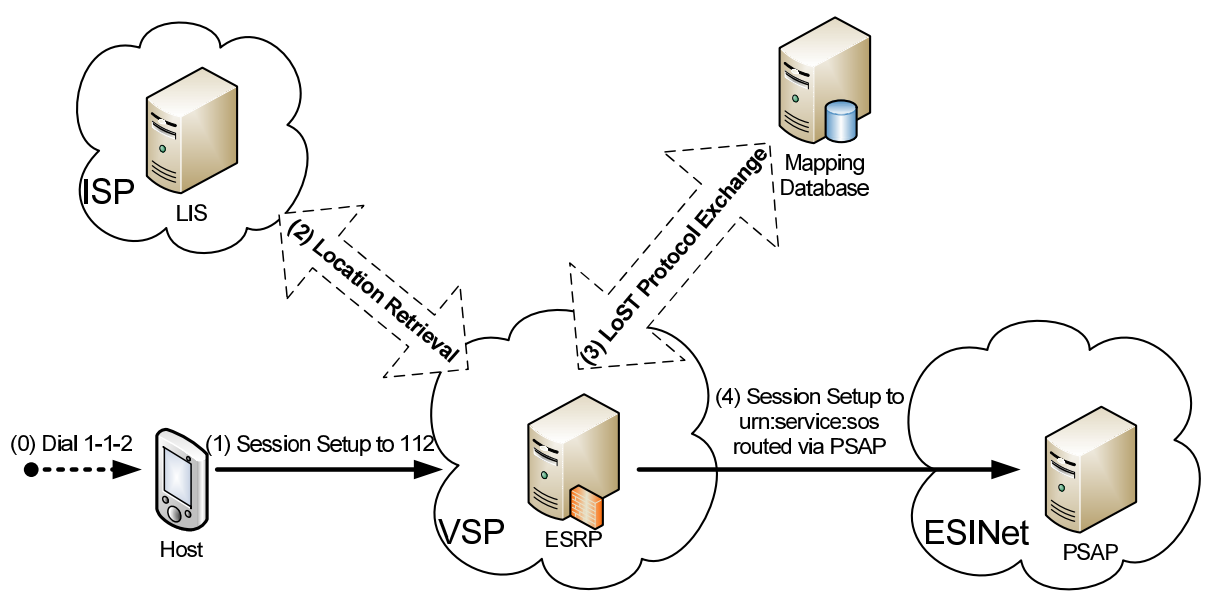

Figure 5.7.: Emergency Services Architecture with Legacy End Points.

There are two main challenges to overcome when dealing with legacy devices: First, the VSP has to discover the LIS that knows the location of the IP-based end host. The VSP is only likely to know the IP address of that device, which is visible in the call signaling message that arrives at the VSP. When a LIS is discovered and contacted, and some amount of location information is available, then the second challenge arises, namely how to route the emergency call to the appropriate PSAP. To accomplish the latter task, it is necessary to have some information about the PSAP boundaries.

[15] does not describe a complete solution for interworking with legacy PSAPs but instead offers building blocks to use. The following constraints exist when dealing with legacy end points:

- Only the emergency numbers configured at the VSP are understood. This may lead to cases where a dialed emergency number is not recognized or a non-emergency call is routed to a PSAP.

- Using the host's IP address to discover the ISP network to which the host is attached is challenging and may, in case of mobility protocols and VPNs, lead to wrong results.

- Security and privacy concerns may arise when many VSPs/ASPs can retrieve location information from an ISP. It is likely that only authorized VSP/ASPs will be granted access to location information for privacy, security and business reasons. Hence, it is unlikely that such a solution would work smoothly across national boundaries.

- When the emergency call is not recognized by the User Agent then functions like call waiting, call transfer, three-way call, flash hold, outbound call blocking, etc. cannot be disabled. This may lead to unexpected user experience.

- The User Agent software may block callbacks from the PSAP since it does not have ways 
to recognize incoming calls related to the earlier emergency.

- Privacy settings may not be respected, and identity information may get disclosed to unauthorized parties. These privacy features exist in some jurisdiction are even available for emergency calls.

- Certain VoIP call features may not be supported, such as a REFER (for conference call and transfer to secondary PSAP) or the Globally Routable User Agent URIs (GRUU) for identifying individual devices issuing the emergency call.

- User Agents will not be able to convey location information to the VSP (even if it is available).

As discussed in Chapter 3 a LIS discovery mechanism has been standardized with RFC 7216 [23] and HELD was extended in RFC 7840 [27] to also return emergency services call routing information. While this helps to tackle the main challenges it still leaves the international deployment questionable due to security and privacy concerns.

\subsubsection{Partially Upgraded End Hosts}

A giant step forward in simplifying the handling of IP-based emergency calls is to provide the end host with some information for LIS discovery. The end host may, for example, learn the ISP's domain name, by using LIS discovery [60], or might even obtain a Location by Reference (LbyR) via HELD [24]. The VSP is then either able to resolve the LbyR in order to route the call or to use the domain to discover a LIS using DNS. It is even possible to associate policy information to the URI resolution procedure based on the extension defined in RFC 7199 [61]. The use of LbyRs together with this policy extension provides additional privacy features since location information is only disclosed to those parties in possession of the location reference and the end devices, as the target, can control the resolution process using policies.

Additional software upgrades at the end device may allow emergency calls to be recognized based on some pre-configured emergency numbers (e.g., '1-1-2' and '9-1-1') and allow for the implementation of other emergency service-related features, such as disabling silence suppression during emergency calls.

\subsubsection{Emergency Services for Persons with Disabilities}

The capabilities of modern IP-based communication protocols, such as SIP, easily enable multi-media communication. Video, real-time text, and instant messaging give persons with 
disabilities access to emergency services. Unfortunately, support for multi-media communication by emergency authorities is still poor, if available at all. Fax has been used in several countries in Europe, and Teletypewriter (TTY) in the US. In the meanwhile, more and more countries are offering SMS-based access to emergency services for persons with disabilities. The European REACH-112 pilot project [62] showed that the standardized IPbased emergency services technology is available and working. Proper integration of the text messaging system into the PSAP software environment is, however, often not available and additional training of call takers is necessary to ensure proper response times. From the REACH-112 pilot and from various SMS-based emergency services deployments, it is known that few persons make use of the new capabilities. This circumstance may have many reasons, such as a lack of awareness of the availability of such a service, unfamiliarity with multi-media emergency calls, and the smaller user base that needs such capabilities (compared to those who feel comfortable just making voice-based emergency calls).

The ability to use multi-media communication has significantly increased over the last 10 years with the increasing capabilities of smart phones. Transmitting instant messages, real-time text, pictures, and video is a common feature of applications running on smart phones and Internet tablets. Since the deployment of IP-based phones is increasing dramatically, we expect that more users, not only those with disabilities, will find the multi-media functionality attractive.

The use of IP-based protocols does not only offer additional functionality but also adds properties like protocol feature and media type negotiation. This allows two communication end points to agree on the latest and best possible communication mechanisms. This is clearly important for extensibility and incremental deployment. If, for example, a device supports a better and newer audio codec then this may lead to better audio quality with a lower bandwidth overhead. In addition to the support provided by SIP, LoST allows emergency services authorities to publish information about the protocols through which they are reachable via information encoded in the URIs returned by the LoST protocol.

\subsubsection{Vehicle Initiated Emergency Calls}

A quick response by emergency services is essential to save lives in a car accident. When an emergency call is automatically triggered, and location information is provided along with the emergency call then the number of road deaths and injuries can be reduced.

In the late 1990s the European Commission started an initiative to require car manufacturers to install a so-called in-vehicle system (IVS), which enables vehicle initiated emergency calls. This system became known as eCall. The IVS is able to initiate an emergency call, 
supports GPS-based location information and can obtain sensor data from the vehicle.

A circuit switched eCall system was standardized, which uses a regular voice call and an inband modem to communicate vehicle information, crash related sensor data, and location information (the Minimum Set of Data or MSD) within the voice channel.

Deploying this circuit switched eCall system took a very long time, mostly due to political reasons. With the transition to IP-based networks it was an obvious step to also standardize the next generation (NG) eCall system. NG-eCall moves from circuit switched to all-IP and carries the vehicle data and crash information as additional data along with SIP signaling.

RFC 8147 [63] standardizes the NG-eCall functionality by re-using the already specified IP-based emergency services functionality. RFC 8147 defines the necessary MIME media types for the MSD and a procedure for requesting and responding to further data requests from the PSAP.

A new new Service URNs is defined in RFC 8147 to allow the routing of eCalls, both manually initiated as well as automatically triggered, to the correct PSAP. A test Service URN is also defined to allow the eCall system to be tested without negatively impacting any ongoing emergency calls.

A separate specification, RFC 8148 [9], specifies emergency calls placed by vehicles for use in regions other than Europe, which make use of a different set of vehicle (crash) data, namely the Vehicle Emergency Data Set (VEDS) rather than the eCall Minimum Set of Data (MSD). In addition to the different data communicated to the PSAP three different deployment models are supported:

- In the "direct model" the IVS communicates the emergency call and the crash data directly to the PSAP.

- In the "paired model" the IVS interacts with a handset, which then communicates the emergency call and the crash data to the PSAP.

- Finally, in the "TSP model" the IVS interacts with a third party, the Telematics Service Provider (TSP), which then routes the emergency call and the crash data to the PSAP.

\subsubsection{Additional Data}

Call takers, first responders, and the emergency services community in general prefer to have better situational awareness about an emergency related incident. More information alone is, however, not sufficient it also needs to be of better quality. The importance of accu- 
rate location information has already been highlighted and with emergency calls triggered by vehicles additional information is available about the crash and the vehicle itself.

There are, however, various other sources of information. The originating device, the access network provider to which the device is connected, and all service providers in the path of the call have information about the call, the caller, or the location, which is helpful for the PSAP to have in handling the emergency.

RFC 7852 [64] defines data structures and mechanisms to convey such data to the PSAP either per-value or by reference. Conveying information by reference allows extra access control policies to be applied before releasing information. This can help to ensure better privacy protection since this additional data about the call, caller, and the location is data directly or indirectly related to a person.

\subsubsection{Data-Only Emergency Calls}

RFC 6443 [57] describes how IP-based emergency calls are initiated from devices using SIP and how they are processed by PSAPs. The focus is thereby on multimedia calls, which almost always includes voice but may include real-time text, video and instant messaging. The ability to include additional data alongside the call has been highlighted previously already.

In some cases devices do not need to establish a communication session to transmit voice, video and other multimedia. [10] focuses on the cases where the transmission of application data is all that is needed. To communicate sensor information the Common Alerting Protocol (CAP) is used inside a SIP MESSAGE. For communication security DTLS / TLS is re-used. CAP is known for authority-to-citizen early warning but can also be used in the reverse communication direction, namely from devices to the PSAP. CAP is an XML-based document format with pre-defined information elements to communicate meta-data about the alert and the sensor readings itself. For location information and other data the existing payloads can be used in the SIP message offering seamless integration of sensors and other Internet of Things devices into the emergency services infrastructure.

The data-only emergency call concept marked a transition from the classical voice (where the human was in the center of the communication) to a model where data is communicated automatically by machines. While this work is still at an early stage it represents a promising direction for future research where automatic processing of many events combined with machine learning may allow to detect dangerous situations quicker. Chapter 7 explores options for securing data communication of IoT devices using standardized security technologies. 



\section{Chapter 6}

\section{Securing IP-based Emergency Services Networks}

\subsection{Introduction}

IP networks and the SIP-based communication infrastructure creates the foundation on top of which emergency services support is added via a number of building blocks. The building blocks provide the basis for (a) determining location information, (b) recognizing an emergency call, (c) evaluating the route towards the PSAP, (d) establishing the SIP session setup and (e) exchanging multi-media data traffic (such as voice, video, text messages, and real-time text). As explained in Chapter 5 there are architectural variants that impact which of these building blocks are used and how. While there are unique aspects to each of these architectural variants, many of the security aspects are still common across all IP-based emergency services deployments.

When considering the security properties of the overall emergency services system, two observations can be made:

1. Emergency services support builds on top of the regular communication infrastructure, and therefore the security properties of the underlying infrastructure are inherited.

2. Security has to be taken into consideration for each and every protocol since the resulting system is as secure as the weakest link. This also implies that the security analysis available for each protocol has to be taken into account by the respective users of those protocols.

Developing protocol specifications by incorporating security during the design is only the first step in developing secure Internet-scale systems. Implementations of these pro- 
tocols also need to be secure against various attacks, such as buffer overflows, and the deployed system must be configured in the appropriate way (e.g., to withstand denial of service attacks or to be secured against unauthorized access). Building secure communication systems that include emergency services components is complex and presumes education and awareness (e.g., via employee training), good software management practices (e.g., secure coding, extensive testing, software update processes), established and working processes (e.g., incident management, clearly defined responsibilities and accountability), etc. A detailed discussion of the secure software development lifecycle is, however, outside the scope of this thesis. Instead, the focus of this work is mainly on the initial phase of the lifecycle, namely on the protocol design. Building communication systems based on standards provides better guarantees that specifications have been extensively reviewed thereby improving the quality of the work.

To focus on the security characteristics that are unique to emergency services I first describe the communication model, the involved entities, and then investigate the adversary model and the security threats. Finally, a list of countermeasures is provided.

\subsection{Communication Model}

The high-level communication models are shown in Figure $6.1^{10}$.

As shown in Figure 6.1, there are four main communication interactions to consider:

I. SIP Signaling Communication: The SIP signaling exchange is at the heart of the IP-based emergency services communication and travels between the endpoints, the end host and the PSAP. SIP [66] is an application-layer control (signaling) protocol for creating, modifying, and terminating sessions with one or more participants. Emergency services functionality has been added to SIP with specifications that define the ability to express emergency services functionality (by using the service URN specification in RFC 5031 [42]) and the ability to carry location objects (see [67]). SIP uses proxies as intermediaries to route the call. Due to the added emergency services functionality SIP roxies

\footnotetext{
${ }^{10}$ This chapter uses the terms 'Location Server' (LS) as well as 'Location Information Server' (LIS). RFC 6280 [65] describes the LIS as follows: "Some entities performing the Location Generator (LG) role are designed only to provide Targets with their own locations, as opposed to distributing a Target's location to others. The process of providing a Target with its own location is known within Geopriv as Location Configuration. The term 'Location Information Server' (LIS) is often used to describe the entity that performs this function. However, a LIS may also perform other functions, such as providing a Target's location to other entities." RFC 6280 defines the Location Server as follows: "Location Server: The Location Server is responsible for enforcing the Target's Privacy Rules."
} 
I.

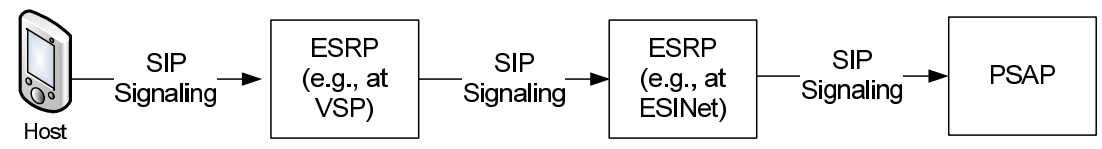

II.

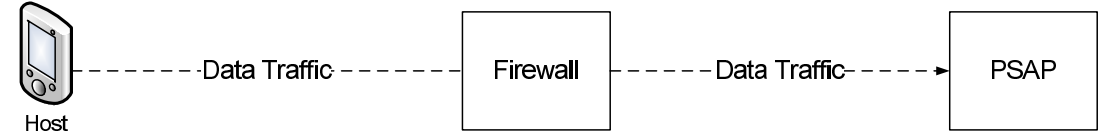

III.
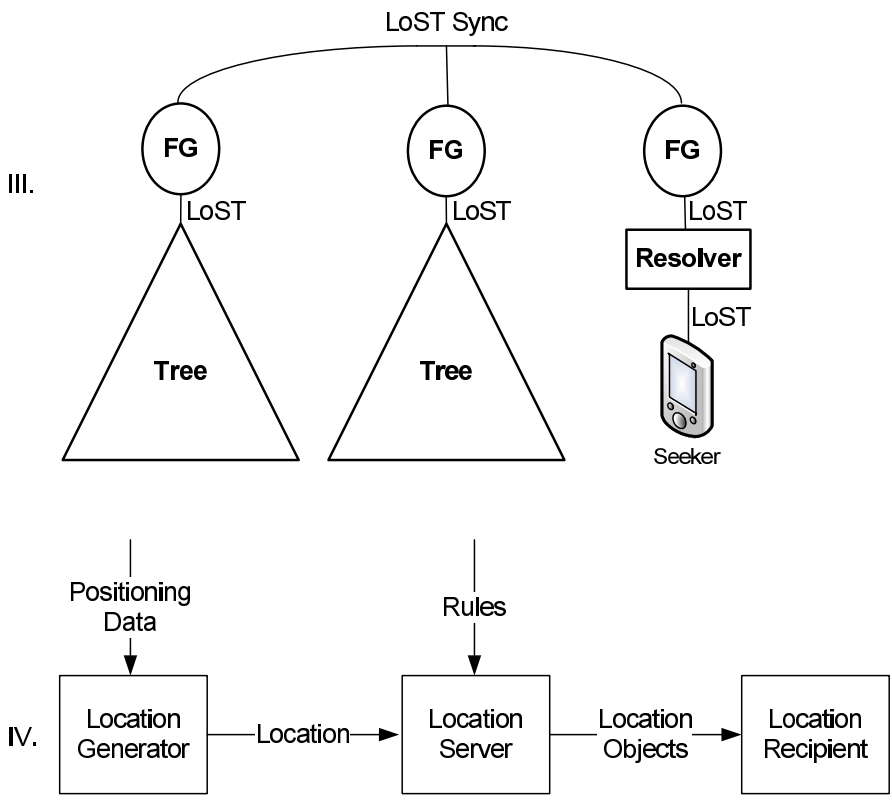

Figure 6.1.: Communication Model Overview.

are also called Emergency Services Routing Proxies (ESRPs). The PSAP itself is located inisde the Emergency Services IP Network (ESINet). Chapter 5 describes the emergency services architecture as standardized by the IETF in more detail. After the emergency services communication is terminated, it is possible for the PSAP to initiate a callback to the emergency call, for example, when details about the situation need to be clarified or the caller prematurely terminates the conversation. The call setup procedure then starts with the PSAP and the SIP session setup messages travel towards the end device that initiated the call.

II. Exchange of Multi-Media Data: The main purpose of the communication setup is to exchange data. For emergency calls this typically takes the form of voice packets. In the future, this will also be video, instant messages, real-time text, and pictures, supported by the capabilities of IP. SIP can detect and negotiate the supported functionality and to enable the exchange of these additional media streams. The used media will largely 
depend on the capabilities of the devices, the installed software applications, and the preferences of the emergency caller. For persons with disabilities, real-time text and video communication is likely to make a big difference, but might require a third party relay service to be invoked, for example, for sign language interpretation.

III. Mapping Database: When emergency call setup is initiated, the call routing processes must decide the next hop to route the setup messages to. A major factor in call routing is location information since the geographically closest PSAP will often want to receive the call first. In some cases, other factors, such as the load situation at a PSAP or the ability to accept certain media types, also influence the decision. For location-based routing, the distributed mapping database that uses the protocol LoST is used, as illustrated in [55] and described in Chapter 5. The design allows end hosts and ESRPs to ask for the appropriate PSAP based on a given location input.

IV. Location Infrastructure: The location retrieval interaction starts with an entity issuing a request for a location object. The requesting party is the Location Recipient, which may be the end host, an ESRP, or the PSAP. Each of these entities have a legitimate interest in receiving location information for the determination of the appropriate emergency dial strings, for emergency call routing, or for the dispatch of first responders. When a location server, which is commonly assumed to be operated by an ISP, receives a location request, it may not always have the current information cached. The location server then starts a location determination process whereby location generators collect positioning data. The location server may provide location information not only as a oneshot transaction, or may provide multiple updates as location determination techniques compute better location over time or when the location of the target device changes. For security and privacy purposes, location may not be shared without a prior authorization check, which is invoked based on a rule set available to the location server. RFC 6280 [65] illustrates a location architecture and introduces terminology.

\subsection{Adversary Models}

In a discussion about security, it is important to keep the anticipated capabilities of an adversary in mind. We distinguish between three types of adversaries:

External Adversary Model: This type of adversary is the most commonly considered adversary in communication networks. The adversary is external to the analyzed system and interferes from the outside. In such a threat model, it is assumed that none of the emergency service infrastructure elements has been compromised. Consider the case where a person makes an emergency call. An adversary could be located along the communication path between the end host and the location server, between the end host and 
the PSAP, or between any other emergency services components. Without proper communication security, this type of adversary could eavesdrop on the communication, inject new messages, or prevent the emergency caller from successfully calling for help.

Malicious Infrastructure Adversary Model: With this type of adversary, we assume that some entities in the emergency services infrastructure are either mis-configured or compromised and can thereby disrupt the normal emergency system operation. Such misconfigured or compromised elements can include the LIS, the Location-to-Service Translation (LoST) infrastructure [52,55], and call routing elements, which may be manipulated to respond with false information.

Malicious End Host Adversary Model: With this adversary model we assume that the end system is compromised. Although this type of adversary model, is a sub-category of the malicious infrastructure adversary model it deserves special attention since end systems, like tablets and desktop PCs, are often more vulnerable due to their poor software update policy and lack of proper administration. Furthermore, a human interacting with the emergency services authorities may have intentions that are not aligned with the call takers or the first responders. We will discuss these problems in context of hoax calls.

In the description of each security threat, the assumptions that need to be met for a successful attack will be described next.

\subsection{Security Threats}

As a starting point we have to think about the motivations of an attacker and to speculate about their available resources to launch such an attack. While some attacks will "carry over" from the existing telephony system, others will be new due to the additional capabilities offered by IP-based emergency services systems.

Attackers may direct their efforts either against a portion of the emergency response system or against an individual. Attacks against the emergency response system have three possible objectives:

- to deny system services to all users in a given area. The motivation may range from thoughtless vandalism, to wide-scale criminality, to terrorism.

- to convey prank calls to PSAP call takers and first responders. A fairly small number of prank calls may consume an enormous amount of resources from the emergency services infrastructure. While there are many variations of these prank and hoax calls a severe version is called "swatting" where an adversary simulates the commission of a punishable offense (such as a hijacking situation) and at the same time fools the emergency services 
system in using wrong location information for dispatching police units. Most of the reported incidents of swatting took place in the US and in Canada [68]. Hoax calls do, however, happen also in other parts of the world, for example in Europe [69].

One can certainly imagine other, more exotic types of attacks. For example, an attacker could

- divert emergency calls to non-emergency sites. This is a form of a denial-of-service attack that can be very confusing for the emergency caller since he or she expects to talk to a PSAP operator but instead gets connected to someone else.

- gain unauthorized access to paid services by using an emergency identifier to bypass regular authentication, authorization, and accounting procedures. The goal of the adversary with such an attack is to gain a financial advantage.

- get elevated priority treatment in the hope to get faster service access.

Attacks against an individual may have the following motivation:

- to prevent an individual from receiving aid.

- to gain information about an emergency that can be applied either against an individual involved in that emergency or to the profit of the attacker.

- to deliver unwanted messages to a non-emergency caller using technologies developed for emergency services purposes. For example, the PSAP callback mechanism [7], if deployed incorrectly, could be used to bypass authorization policies.

- to use technology designed for emergency services to turn a user's phone into a recording device.

- to use sensitive personal data (e.g., health records) for non-emergency services purposes, or to retain personal data beyond an acceptable retention period.

- to disclose information about the call, caller, and the emergency situation to third parties without prior consent of the emergency caller.

In the subsections below we describe a few attacks in more detail. The list is not exhaustive but illustrates concerns frequently raised.

\subsubsection{Denial of Service Attacks}

Emergency services have at least three finite resources subject to denial of service attacks: the network and server infrastructure, call takers, and first responders, such as fire fighters 
and police officers. The task of protecting network and server infrastructure shares similarities with high-value e-commerce sites, and lessons can be learned from these environments particularly regarding capacity planning, load balancing, DDoS prevention techniques, and abuse reporting. Call takers are a far more limited resource; even large cities only have PSAPs with only a handful of PSAP call takers on duty. Even if the call takers attempt to question the caller to weed out prank calls, they would be quickly overwhelmed by even a small-scale attack. Finally, first responder resources are limited as well; and since every assignment takes an extended period of time, their resources are quickly dried up.

\subsubsection{Attacks Involving the Emergency Identifier}

The overall process of establishing an emergency call begins with the person in need for help dialing the emergency dial string. The exact sequence of digits depends on the infrastructure the device is connected to. Although 1-1-2 became the emergency services number for Europe, and 9-1-1 for the US, many countries still provide emergency numbers in addition to the 112/911. Furthermore, many large enterprises, universities, and hotels prefix the emergency numbers with additional digits, such as 0-112. Therefore, it is important for devices that can be used in different environments to automatically detect which dial string triggers an emergency call. Pre-programming the list of numbers in use world-wide is not possible due to the overlap of emergency and non-emergency numbers. Attempts to agree on a smaller set of emergency numbers has not been very successful so far.

With dial strings there are two challenges to solve:

1. A device needs the ability to learn the emergency services numbers available for a specific attachment point. LoST provides a mechanism for obtaining the emergency dial string for a given location. 3GPP radio networks also allow a device to be provisioned with emergency numbers.

2. For unambiguous processing of protocol messages throughout the emergency call chain, it is useful to replace the user entered dial string with a symbolic name. This is accomplished with the service URNs, see RFC 5031 [42]. Calls marked with this emergency identifier are then treated as emergency calls by the call routing entities. An example of a service URN is "urn:service:sos.police". In combination with LoST's ability to learn the dial string for a given location, this allows a device to dynamically translate emergency dial strings to service URNs for usage in emergency calls.

However, users do not "dial" an emergency URN themselves. Instead, the entered emergency dial strings are translated to corresponding service URNs and then these service URNs are carried in SIP signaling messages (e.g., the Request-URI of a INVITE request). 
This translation should ideally be done at the end point so that the semantic of the emergency call is preserved throughout the entire end-to-end signaling chain. This allows location information information to be added by the end host or by intermediaries along the signaling path and to enable or disable emergency services relevant call features (e.g., noise cancellation). Also, once a call is marked with a service URN call routing entities give it preferential treatment.

The main possibility for an attack involves use of the emergency identifier to bypass the normal procedures in order to achieve fraudulent use of services. An attack of this sort is possible only if the following conditions are true:

- The attacker is faking an emergency call.

- The call enters the domain of a service provider, which accepts it without applying normal procedures for authentication and authorization because the signaling carries the emergency identifier.

- The service provider routes the call according to the called address (e.g., SIP RequestURI), without verifying that this is the address of a PSAP (noting that a URI by itself does not indicate the nature of the entity it is pointing to).

If these conditions are satisfied, the attacker can bypass normal service provider authorization procedures for arbitrary destinations, simply by reprogramming the emergency caller's device to add the emergency identifier to non-emergency call signaling. If resource priority headers [70] are used, a call routing device may also be tricked into using this information as the sole basis for bypassing authentication, or authorization procedures if it uses these headers as the sole basis for its decision. This would also allow an adversary to use this indication to gain preferential treatment of marked traffic over other network traffic.

\subsubsection{Attacks Against the Mapping System}

This section considers attacks intended to reduce the effectiveness of the emergency response system for all callers in a given area. If the mapping operation is disabled, then the correct functioning of the emergency call routing infrastructure cannot be guaranteed. Consequently, the probability that emergency calls will be routed to the wrong PSAP increases. Routing calls to the wrong PSAP may have two consequences: emergency response to the affected calls is delayed, and PSAP call taker resources outside the immediate area of the emergency are consumed due to the extra effort required to redirect the calls. Alternatively, attacks that cause the client to receive a URI that does not lead to a PSAP have the immediate effect of causing emergency calls to fail. 
Three basic attacks on the mapping process can be identified: denial of service, impersonation of the mapping server, or corruption of the mapping database. Denial of service can be achieved in several ways:

- by a flooding attack on the mapping server;

- by taking control of the mapping server and either preventing it from responding or causing it to send incorrect responses;

- by taking control of any intermediary node (for example, a router) through which the mapping queries and responses pass, and then using that control to block them. An adversary may also attempt to modify the mapping protocol signaling messages. Additionally, the adversary may be able to replay past communication exchanges to fool an emergency caller by returning incorrect results.

In an impersonation attack, the attacker induces the mapping client to direct its queries to a host under the attacker's control rather than the real mapping server, or the attacker suppresses the response from the real mapping server and sends a spoofed response.

The former type of impersonation attack itself is an issue of mapping server discovery rather than the mapping protocol directly. However, the mapping protocol may allow impersonation to be detected, thereby preventing acceptance of responses from an impersonating entity and possibly triggering a more secure discovery procedure.

The details of the attack surface may, however, vary between the different designs. More details about LoST are provided in the subsequent paragraph to illustrate some of the design decisions.

For those cases where an end host obtains the LoST server URI via DHCP, as described in RFC 5223 [56], it has to rely on the security of the local network and the mechanism to protect network access since there is no usable DHCP security mechanism available today. While there is a sufficiently solid security infrastructure in most enterprise networks the situation is very different in hotspots and home networks where either no network access authentication is provided or configuration is managed by ordinary end users.

In other deployments the DNS infrastructure will be used to discovery a LoST server. It is worthwhile to highlight a design decision that was made for the discovery procedure, which uses the Dynamic Delegation Discovery Service (DDDS) described in RFC 3958 [71]. Contrary to the advice given in RFC 3958 where the input to the discovery procedure is the reference identifier, the discovery procedure specified in Section 4 of RFC 5222 uses the output of the discovery procedure as the reference identifier. The reference identifier is defined in RFC 6125 [72] to describe an algorithm for verifying the service identity. This 
approach introduces additional security risks because the attack surface is extended to the DNS infrastructure. The attacks include, for example, interception of DNS packets between the client and the recursive name server, DNS cache poisoning, and intentional modifications by the recursive name server; see RFC 3833 [73] for more comprehensive discussion. This design decision was made to make LoST deployment practical because many application service providers will not operate their own LoST servers but will instead delegate operation to an existing LoST server infrastructure. As such, there will be a large number of application service providers using a relatively small number of LoST servers. A given LoST server may not even be aware of all the application service domains pointing to it.

Another concern with the use of a distributed mapping database is the ability to attack the mapping database itself. Injecting fake mapping entities into this distributed mapping database may lead to an inconsistent state, and could also lead to denial of service attacks. If the mapping data entry contains a URL to a server that does not exist then emergency services for the indicated area are not reachable. If the URL for many (or all) mapping data entries point to a single PSAP (rather than to the original envisioned, possibly larger number of PSAPs) then this PSAP is likely to experience overload conditions. If the mapping data contains a URL that points to a server controlled by the adversary itself, impersonation is possible.

\subsubsection{Attacks against the Location Information Server}

A LIS provides location information to end hosts and other entities in the system, which is then used for routing of emergency calls and for dispatching first responders. The LIS itself often has to obtain information from other sources to determine the location of an end host. The complexity of this process varies with the size of the network topology, with the used network infrastructure (e.g., fixed vs. mobile technology), the mix of different technologies (e.g., WLAN and cellular radio technologies), the features supported by end devices to cooperate in the location determination procedure. More details about this process can be found in RFC 6280 [65].

An adversary who wants to provide false location to a PSAP has a number of choices. Tampering with location information is one possibility and interfering with the location determination procedure executed by a LIS is another possible approach.

The process of determining location information heavily depends on the specific network deployment, but at an abstract level, the process is simple: A Location Recipient, like the end host or an ESRP, transmits a request for location information to a LIS. Some information about the device to be located has to be conveyed in the request to allow the LIS to perform 
the location lookup. Unfortunately, there is no unique device identifier available. Location Recipients have a few identifiers to choose from; the IP address is commonly used. Other identifiers are, as defined in [25], MAC address, Network Access Identifier, and a DHCP Unique Identifier. When a LIS receives such a request for location information, it associates the obtained identifier with information available in its databases. The data may have been manually provisioned, but will typically be collected automatically from normal network operation, such as network management, network attachment procedures, mobility protocols, and security protocols. For example, a LIS located in a DSL network receives a request asking for location related to a specific IP address. The IP address may be allocated from a pool of addresses maintained by the Authentication, Authorization and Accounting (AAA) server and therefore the AAA server has to be queried. The AAA server is used during the network access authentication procedure to authenticate the end host and the user and authorizes the use of certain resources on the network. Examples of AAA technologies are RADIUS [74] and Diameter [75]. The AAA server may know the current attachment point of the end host or may use available information about the DSL Access Module (DSLAM) and Access Node (AN) and the related identifiers (e.g., Ethernet VLAN tags, Layer 2 tunnel identifiers, virtual port ID (VPI) and virtual circuit ID (VCI)) to determine the position of the end host. Further examples of such measurement identifiers are provided in [76].

Consequently, there are various methods by which an adversary can interfere with the process of resolving a chain of identifiers to obtain location information. If the adversary succeeds in feeding incorrect information in the lookup step, it may be able to fool a LIS into providing out wrong location information. Examples of interfering with identifier mapping include the sending of a false MAC address or an IP address to obtain different location information. It should also be noted that wiremap maintenance is prone to errors thereby resulting in wrong information being provided out even in the absence of malice.

Caller ID spoofing in today's network is a common way to trick the location lookup procedure into producing the wrong result. Details are described in [77].

\subsubsection{Swatting}

Prank calls have been a problem for emergency services, dating back to the time of street corner call boxes. Individual prank calls waste scarce emergency service resources and possibly endanger bystanders or emergency service personnel as they rush to the reported scene of a fire or accident. Risks to human life are not a typical security threat within communication protocols. Emergency services are, however, different in this regard. 9-1-1 'swatting' incidents with life threatening consequences have captured media attention [78]. Some of these incidents have involved spoofing of the originating phone number when 
calling 9-1-1, leading to a false location being obtained via the phone number-to-location lookup used in fixed line environments. This could result in a SWAT team being dispatched to the location of a completely innocent citizen if the swatter is able to convince the call taker that a serious crime is under way. The FBI has warned about the increasing prevalence of swatting incidents [79].

To reduce prank calls the legacy emergency service infrastructure relies on the ability to identify callers and on the difficulty of location spoofing for ordinary users. To ascertain the caller's identity is important since the threat of severe punishments reduces prank calls. Mechanically placing a large number of emergency calls that appear to come from different locations has believed to be difficult but has gotten increasingly easier with the use of VoIP, the ability to get illegal access to the backend telecommunication infrastructure, new business models (such as bulk unsolicited commercial messaging), certain features of the telecommunication infrastructure (such as the lack of cryptographic validation of accurate caller origination), and the increasing number of VoIP providers that make difficult to trust everyone of them. Calls from pay phones are subject to greater scrutiny by the call taker. In the PSTN, it was more difficult for an attacker from one country to attack the emergency services infrastructure located in another country.

In countries that do not allow SIM-less legacy emergency calls the identity of most callers can be ascertained, so that the threat of severe punishments reduces prank calls. The term SIM-less emergency calls refers to the use of mobile phones without a SIM card where no authentication takes place at the network and at the application layer. The term was introduced when mobile phones did not run third party VoIP applications that could also be used to make emergency calls. As a comparison, in countries where SIM-less emergency calls are allowed prank calls may be as high as 50\% [69].

There is a fear that VoIP systems further simplify these types of prank calls when identities and location information can easily be crafted. It may even be possible to have attackers located in one country to attack the emergency services infrastructure located in a different country or to mechanically (with the help of bot nets) initiate a large number of emergency calls that appear to come from different locations.

\subsubsection{Attacks to Prevent a Specific Individual from Receiving Aid}

If an attacker wishes to deny emergency service to a specific individual, the mass attacks described earlier will also work provided that the target individual is within the affected population. The collateral damage would, however, be huge. Except for the flooding attack on the mapping infrastructure, the attacker may also want to focus on a specific individual. 
To guarantee effectiveness an adversary may attack the end device directly rather than the emergency services infrastructure.

The choices available to the attacker are

- to take control of any intermediary node (for example, a WLAN router at the user's home). Absent further security mechanisms this allows the adversary to modify requests and responses or to even block the entire communication. Improper configuration or lack of software updates leaves many home routers vulnerable to attacks, as these incidents demonstrate [80,81].

- to interfere with the communication of the end device and other emergency service entities, for example, over the WLAN home network.

- to infect the user's device with malware and consequently to have full control over the device.

In general, these type of attacks are difficult (or impossible) to prevent by an emergency services system itself since they rely on the overall security of the Internet eco-system in general.

\subsubsection{Attacks to Gain Information about an Emergency}

This section discusses attacks used to gain information about an emergency. The attacker may be seeking the location of the caller (e.g., to effect a criminal attack) or to use information to link an individual (the caller or someone else involved in the emergency) with embarrassing information related to the emergency (e.g., "Who did the police take away just now?"). Finally, the attacker could take profit from the emergency, perhaps by offering his or her services (e.g., a news reporter, or a lawyer aggressively seeking new business). The primary information that interceptions of mapping requests and responses will reveal are a location, a URI identifying a PSAP, the emergency service identifier, and the addresses of the mapping client and server. The location information can be directly useful to an attacker if the attacker has high assurance that the observed query is related to an emergency involving the target. The type of emergency (fire, police, or ambulance) might also be revealed by the emergency service identifier in the mapping query. The other pieces of information may provide the basis for further attacks on emergency call routing. The attacker may gain information that allows for interference with the call after it has been set up or for interception of the media stream between the caller and the PSAP.

Finally, the attacker may gain access to the conversation between the emergency caller and the call taker rather than just the meta-data about the incident. This is particularly easy 
if the media communication is not confidentiality protected.

\subsubsection{Interfering with the LIS and LoST Server Discovery Procedure}

Many entities in the emergency services architecture are configurable to offer some amount of flexibility. Dynamic discovery procedures have been developed to avoid manual configuration. The LIS and the LoST server discovery are examples.

The primary attack against the discovery step is impersonation. When there is no natural a-priori relationship between the two devices, then the attack surface is increased. End devices, for example, are not supposed to be pre-configured manually with a LIS located in their ISPs network. Particularly, for mobile devices that frequently change their point of attachment the LIS needs to be dynamically discovered. In case of LoST, however, an end device may be statically provisioned to use a single LoST server all the time. Similarly to the DNS, however, it is possible to discover and use a local LoST sever, which would provide improved resilience and shorter round-trip times.

An attacker could attempt to compromise LIS and LoST discovery at any of three stages:

1. providing a falsified domain name to be used as input to U-NAPTR (for example when this information is provided via DHCP to the end devices $[56,60])$,

2. altering the DNS records used in the U-NAPTR resolution $[52,82]$,

3. impersonating the LIS or LoST server itself.

The U-NAPTR resolution process is entirely dependent on its inputs. In falsifying a domain name, an attacker avoids any later protections, bypassing them entirely. To ensure the reliability of the access network domain name DHCP option, it is necessary to prevent DHCP messages from being modified or spoofed by attackers.

Once a client has been tricked into talking with the wrong LIS or LoST server, subsequent steps for emergency service protocol execution may fail or are manipulated in favor of the adversary.

\subsubsection{Call Identity Spoofing}

If an adversary can place emergency calls without disclosing its identity, then determining the source of prank calls is more difficult. Neither the PSAP call takers nor the emergency 
services authorities authenticate emergency callers themselves directly. There are at least two separate layers of authentication and authorization:

- Authentication at the link layer or at the network layer (e.g., using the Extensible Authentication Protocol (EAP) [83])

- Authentication at the application layer, for example at the VoIP application.

Note that this split is the result of the separation between the ISP and the VSP. Since two different stakeholders manage the identifier space, the identities used during authentication are different as well. While not all architectures assume such a separation of roles, they are nevertheless common on the Internet today.

Even with proper authentication at the VSP or the ISP, there is the question of how strong the prior identity proofing step was, that is, what identity information did the customer provide in order to create an account with an ISP or VSP to use their communication servers. The quality of identity proofing must not be ignored since it links the digital identity to the identity of a person in the real world. This aspect is described in more detail in NIST Special Publication 800-63 [84].

In case of misuse, the emergency services authorities must contact the VSP and/or the ISP to identify a particular individual. In certain cases, there is no authentication procedure executed and hence this re-identification can be challenging. This might, for example, be the case with an open IEEE 802.11 WLAN hotspot. While the owner of the WLAN hotspot can be determined, this may be insufficient for determining the adversary misusing the open WiFi network.

Given the importance of authentication for ensuring accountability in case of misuse, mistakes made in the legacy telephony network can be fixed. The ability to make emergency calls without any form of authentication or by utilizing caller-id spoofing can be prevented by various technical means, education about the negative side-effects, and by regulatory mandates. Mandating authentication for emergency calls, and even the introduction of special credentials, for example an emergency certificate, is imaginable. Dedicated security mechanisms increase costs, introduce an administrative overhead and are only, from a security point of view, useful when widely used. 


\subsection{Countermeasures}

In the previous section we illustrated a few attacks on the emergency services system. In this section, we highlight a number of techniques to mitigate these threats.

\subsubsection{Discovery}

Link layer security is commonly used for securing the initial communication link between an end device and the network infrastructure to reduce the possibility of attack, particularly at the early phases of the communication establishment. Link layer security is particularly useful in those cases where location information is directly exchanged via DHCP, as described in Chapter 5. While DHCP offers its own security mechanism, see RFC 3118 [85], it is impractical for most deployments. However, for the interaction with HELD or LoST, additional security capabilities are available at higher protocol layers since these protocols run over HTTP.

LoST and HELD intentionally share a very similar discovery mechanism that can be used by end devices as well as by intermediate entities like emergency services routing proxies.

In the case of dynamic discovery of a LIS and a LoST server in the access network, an end device performs the following steps:

1. Acquire the access network domain name. DHCP, for example, can provide the end host with this information.

2. Use this domain name as input to the DNS-based resolution mechanism. For LoST, this resolution mechanism is described in RFC 5222 [52]. For HELD, it is defined in RFC 5986 [60]. In both cases, the URI-enabled NAPTR specification [82] is used.

An ESRP may also need to discover the network domain name for a LIS (for example in transition scenarios where no Location by Reference has been made available to the end host or is not included in the call setup procedure by the end host). The ESRP cannot rely on DHCP for discovery of a LIS but instead has to use the IP address provided during the call setup procedure. Reverse DNS look-ups have been proposed for this purpose. For a LoST server discovery, the ESRP is pre-configured with a domain name, which simplifies discovery because every LoST server is able to receive a correct answer from the distributed mapping database, as explained in Chapter 5.

To avoid an attacker modifying the query or its result of any interaction with a LIS or 
a LoST server, Transport Layer Security (TLS) is strongly recommended. Because LoST supports caching of responses, cache poisoning can be particularly problematic.

The entity interacting with a LoST server or a LIS has to check the TLS server's identity, as described in Section 3.1 of RFC 2818 [86].

Consider two independent application service providers called foo.example.com and bar. example.com. They want to use a third party LoST service operated by an emergency organization. This LoST server is reachable at https://lostserver.example.org. foo. example.com includes an additional entry into the DNS server, as shown below:

foo. example.com.

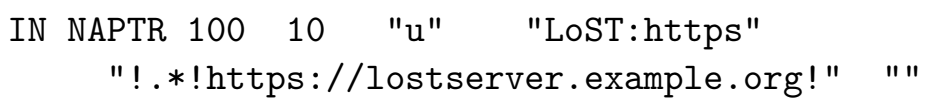

The DNS entry for bar . example.com is:

bar. example.com.

IN NAPTR 10010 "u" "LoST:https"

"!.*!https://lostserver.example.org!" ""

When a LoST client establishes a TLS connection with https://lostserver. example.org it would see a certificate that contains lostserver.example.org in one of the many fields of a certificate (such as CN-ID, DNS-ID, SRV-ID, or URI-ID). The main difference between these four types of certificate fields is the amount of information embedded in the certificate and where. For the two application service providers the additional overhead of provisioning clients is kept at a minimum in such a model. To provide the LoST client with high assurance that https://lostserver.example.org is indeed offering LoST services on behalf of the two application service providers secure DNS has to be used. Without secure DNS an adversary can modify the DNS response and change the LoST server URI without the LoST client noticing it because the LoST client will use the output of the discovery procedure as the reference identifier in the rules for checking the certificate outlined in RFC 6125.

To avoid a dependency on secure DNS deployment an application service providers has two options. We assume that user-configuration is not a viable option because configuration failures may only be recognized during the service usage, which could be fatal for emergency services. 
As a first option, the application service provider can provisioning its clients with the LoST server URI rather than going through a layer of redirection via the DDDS discovery procedure. This is a useful approach when the VoIP/multi-media application is provided by the application service provider and frequent updates are common.

As a second option, it is possible for the application service providers and the organization hosting the LoST server to delegate credentials. Fortunately, the ability to deal with such deployment environments is not new and security solutions have been developed to lower the attack surface (without having to wait for widespread Secure DNS deployment). Domain Name Associations (DNA) [87] and PKIX over Secure HTTP (POSH) [88] are two solutions developed for the Extensible Messaging and Presence Protocol (XMPP). Hosting environments show very similar characteristics to the scenario described above, namely

1. Certification Authorities (CAs) will not issue certificates for foo.example.com and bar . example.com to an organization operating lostserver. example.org.

2. foo.example.com and bar.example.com will not obtain certificates and private keys and hand them over to the entity operating lostserver. example.org.

3. The organization operating lostserver . example. org does not want to manage certificates for foo.example.com, bar. example.com and potentially hundreds or thousands of application service providers due to the added administrative overhead and increased security risk.

While DNA and POSH have not been developed and standardized for use with LoST, their work can be applied to LoST deployments. The following description outlines a proposal for how POSH could used to secure the re-direction problem. In addition to the DDDS discovery of the LoST server URI the LoST client has to perform an HTTPS GET request at the source domain to the path "/.well-known/posh/servicedesc.json". For the LoST service the application-specific string for servicedesc could be "lost". A LoST client using the application service provider foo. example. com would therefore send the HTTPS GET request shown here.

GET /.well-known/posh/lost.json HTTP/1.1

Host: foo.example.com

The following snippet shows an example response (taken from RFC 7711). The fingerprint element contains base64 encoded hashes of an X.509 certificate in DER format. These fingerprints are subsequently used for checking the server certificate provided by the LoST server running on https://lostserver . example.org during the TLS handshake. 


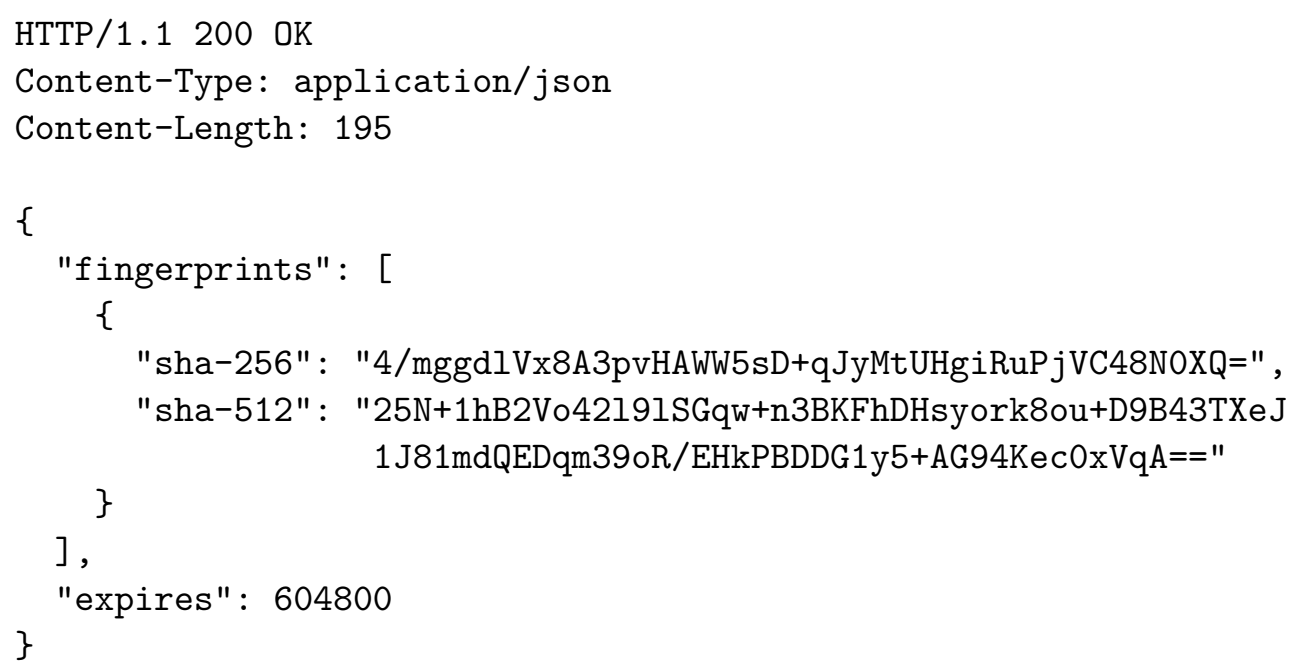

A HELD or LoST server that is identified by an "http:" URI cannot be authenticated. Use of unsecured HTTP does not meet requirements in HELD and LoST. If an "http:" URI is the product of discovery, this leaves devices vulnerable to several attacks. Lower layer protections, such as layer 2 traffic separation might be used to provide some security guarantees against adversaries on the wired-interface, but should not be seen as a replacement for robust, cryptographic protection.

For use of LoST on the public Internet, LoST servers will not need to authenticate or authorize LoST clients presenting mapping queries. For LoST deployments in closed systems, authentication of the underlying transport mechanism, such as HTTP basic and digest authentication, may be used. Basic authentication should only be used in combination with TLS. The usage of TLS with mutual certificate-based authentication is another option, particularly for use between server-to-server communication. Best practices for use of TLS are provided in [89].

\subsubsection{Secure Session Setup and Caller Identity}

The Session Initiation Protocol (SIP) [66] and the Session Description Protocol (SDP) [90] are used to set up multimedia sessions or calls. SIP messages travel from the emergency callers device via the intermediate SIP infrastructure towards the call takers device at the PSAP.

The SIP protocol suite offers solutions for securing SIP signaling as well as conveying caller identity information to the called party. 
The mechanisms can be clustered into three categories:

1. the process of verifying the user's identity. The VSP's infrastructure elements authenticate the user. This can, for example, happen via the basic SIP authentication mechanisms (such as digest authentication).

2. the process of asserting the previously verified identity to a third party. The authenticated identity is not only important for the VSP but also for end-to-end communication to the remote party. The VSP can assert this identity towards other parties using mechanisms such as SIP Identity, described in RFC 4474 [91], or P-Asserted-Identity, specified in RFC 3325 [53].

3. SIP signaling security. This ensures that an adversary cannot inject fake signaling messages, eavesdrop on the communication, replay messages, etc. Transport Layer Security (TLS) is used for providing authentication, integrity, and confidentiality protection between neighboring SIP nodes. Since SIP signaling supports multiple transport protocols, not just TCP, Datagram TLS [92] was introduced to allow TLS functionality to datagram transport protocols.

There are two main technologies for communicating identity information in SIP:

P-Asserted-Identity (RFC 3325): After authenticating the user, the VSP's SIP proxy adds the P-Asserted-Identity (PAI) header to the SIP message. This header carries the authenticated identity (SIP URI) of the user. The P-Asserted-Identity header is protected only in a hop-by-hop fashion between the SIP proxies along the path. The mechanism can only be used within a trust domain in which the SIP proxies and UAs communicate securely and the proxies are mutually trusted. The design of PAI is therefore based on a chain of trust rather than on a cryptographic end-to-end security solution.

SIP Identity (RFC 4474 and RFC 8224): SIP Identity extends the PAI concept with a cryptographic identity assurance. SIP messages are sent to an Authentication Service, which is responsible for verifying that the user agent software knows a shared secret, for example, using the HTTP Digest authentication protocol. Based on successful user authentication, identity information is written into the From header of the SIP request. This part is identical to the PAI scheme. Then, the Authentication Service adds a digital signature to a new SIP Identity header before forwarding it to the final recipient. Within the forwarded SIP request, the Authentication Service also provides a reference (using an HTTP URI in the Identity-Info header) to its own domain certificate. The recipient of the SIP message, for example the call taker's SIP user agent software, performs the following actions to verify the authenticated identity: First, it fetches and validates the certificate of the Authentication Service. Then, it verifies the signature of the SIP message and the identity of the user. Finally, it checks the value of signed Date header to protect against replay attacks. 
RFC 4474 provides identity assurrances only for domain names (and not for telefone numbers), and it does not tolerate intermediaries, like Session Border Controllers, to alter fields in the SIP call signaling. Telephone numbers are still in widespread use today and Session Border Controllers are also frequently deployed to offer policy and interworking functions at network boundaries. A more detailed description of the limitations of RFC 4474 can be found in Section 5.2 of RFC 7340 [77] (and in the initial publication discussing the challenges [93]). For this reason RFC 8224 [94] was written providing an alternative, but conceptually similiar, solution. RFC 8224 together with RFC 8225 [95], which defined the Personal Assertion Token (PASSporT), allow an Authentication Service to create a signed assertion that includes information about the caller, called party, hashes of keys exchanged for DTLS-SRTP [96,97], algorithm information, and SIP Date header. PASSporT is an assertion based on the JSON Web Token (JWT) [98] originally developed for use with the OAuth 2.0 authorization framework [99]. To demonstrate authority over telephone numbers extensions to X.509 certificates where defined in RFC 8226 [100]. These extensions allow indicating the range of phone numbers an entity has authority over.

\subsubsection{Media Exchange}

The main goal of the communication establishment explained in the previous section is in the exchange of multi-media data between the two (or more) SIP end points. The offered security services must not only protect the communication setup but also ensure the protection of the media exchange. SDP, which is responsible for negotiating the media, is a versatile protocol. SDP not only allows the setup of Real-Time Transport Protocol (RTP) [101], which is used to transmit real-time media on top of UDP and TCP [102], but also allows the setup of TCP [103] and additionally TCP/TLS connections for use with media sessions [104].

The Secure RTP (SRTP) [105] is the established standard for securing RTP. In order to allow SRTP to offer its service, cryptographic keys need to be established between the involved communication parties via a key exchange protocol. Various key exchange protocols have been proposed and analyzed [106], and among all the options, DTLS-SRTP, described in RFC 5763 [96,97], was chosen as the preferred IETF mechanism. However, the precursor to DTLS-SRTP, the Security Description (SDES) protocol [107], is widely used today despite its inferior security characteristics.

In order to protect against a number of attacks, it is therefore necessary for the SIP communication end points to implement and use a key exchange protocol. DTLS-SRTP should be used but for backwards-compatibility with older systems SDES) must also be imple- 
mented.

\subsubsection{Mapping Database Security}

Chapter 5 the use of the LoST protocol as part of the distributed mapping architecture is described and LoST Sync allws to distribute mappings between LoST server nodes.

Exchanging mapping information is a security sensitive task. A minimum requirement is to authenticate neighboring server nodes using available HTTP security mechanisms, such as HTTP Digest [108], HTTP Basic [108] over TLS, or plain TLS with client and server certificates [109].

The setup of the LoST server relationships requires some manual configuration and hence the choice of the security mechanisms used between the two entities is a deployment specific decision. Nevertheless, the usage of certificates is an attractive option since it allows the use of a Public Key Infrastructure (PKI) with trust anchors dedicated to the emergency services. Whenever a new server is add a new certificate is obtained from the corresponding Certification Authority (CA). This certificate is then ready for use by the other infrastructure elements without additional administrative configuration burden. In any case, the two communicating end points must authenticate each other and utilize the established secure communication channel (i.e., an integrity protected exchange of data with the help of the TLS Record Layer) to avoid the possibility of injecting bogus mappings.

A malicious entity could, however, intentionally modify mappings or inject bogus mappings. To avoid one entity claiming a service boundary belonging to some else, any node introducing a new service boundary must digitally sign the mapping and thereby protect the data with an XML digital signature. This ensures that a new mapping is associated to a particular owner with non-repudiation properties. Absent any automatic procedures, a system administrator must approve the received mapping prior to its inclusion in the database. Determining who can speak for a particular region is inherently difficult unless there is a small set of authorizing entities that all other participants can trust. Receiving systems should be particularly suspicious if an existing coverage region is replaced with a new one containing different contact points. With this end-to-end security mechanism, it is nevertheless guaranteed that mappings are modified by servers forwarding them as part of the synchronization procedure. 


\section{Chapter 7}

\section{Securing Data-Only Emergency Calls}

\subsection{Introduction}

The Internet allows endpoints to communicate a variety of information through the network without any changes to the underlying infrastructure. The work on IP-based emergency services was initially focused on making voice communication functioning well. Later the attention was shifted to other forms of communication, including real-time text, video and real-time text. Even more recently is the idea to enhance emergency services communication with data collected by Internet of Things devices. In the emergency services community the term "data-only emergency call" is used to refer to devices that typically send data, not voice or not just voice, to PSAPs to help facilitate emergency services support. The communicated information may be crash data of a vehicle, data from a smoke sensor, water level information, structural information of a bridge, etc. In general, the idea is to increase situational awareness for the emergency services personnel and other interested parties (such as the owner of a building).

Over the last few years, the number of Internet of Things (IoT) deployments has seen a steady increase. Heterogeneous, innovative IoT hardware is being rolled out at a fast pace, catalyzed by the new availability of open-source, general-purpose, embedded IoT software and open standards for IoT communication.

In parallel, alarming recent reports in both academic work and mainstream media warn about potential cyber-vulnerabilities and actual cyber-attacks involving IoT. Increased awareness of the need for improved security is pushing legislators to pay closer attention to the IoT environment and issue new regulations, such as the first IoT cybersecurity law (SB-327) and, more recently, the European Commission's Cybersecurity Act. 
In this chapter, we focus on constrained IoT devices (described in RFC 7228 [110]). Typically, constrained IoT devices use microcontrollers - for instance Arm Cortex-M - on which run real-time operating systems, such as FreeRTOS, Micrium's $\mu \mathrm{C} / \mathrm{OS}$, RIOT, or Mbed OS [111]. Compared to machines that run full-blown operating systems, such as Linux, constrained IoT devices use a fraction of the power and are equipped with RAM and Flash sizes (in the kilobyte range) reminiscent of the days of the Commodore VIC-20. Constrained IoT devices, which cannot afford the energy drain of Wi-Fi, connect to the network via low-power, wireless, link-layer technologies, such as Bluetooth Low-Energy, IEEE 802.15.4, LoRa, 3GPP Cellular IoT (NB-IoT), or via wired buses.

IoT devices are, in many case, purpose built devices.

Although EU initiatives, such as IoT security recommendations from ENISA [112] ${ }^{11}$ pave the way for more secure IoT, reports about utterly insecure IoT devices keep pouring in.

In 2014 [2] we surveyed standardization activities on Internet of Things. Most standardization activities were only at the beginning and, in other cases, concepts were not yet ready for standardization and required further research. At that time multi-hop mesh networking using IEEE 802.15.4 and data compression over this low power networking technology received a lot of attention.

5 years later, in 2019, we were wondering whether it is in the meanwhile possible to develop an IoT product, such as a device used for emergency services, and secure it with open standards. While there are still lots of proprietary technologies are on the market, analysing the situation with open standards is beneficial for two reasons: first, the use of standards reduces or avoids vendor lock-in, and second the specifications are publically available.

As a metric for a secure IoT product the ENISA recommendations are used. Following the earlier survey the focus is on Internet protocol specifications for network security standardized by the IETF. The focus on the IETF is motivated both because it has been successful in standardizing many other Internet protocols that underpin the Internet at large, and because IETF protocols standards aim to work across the various IoT link-layer technologies.

\footnotetext{
${ }^{11}$ European Union Agency for Network \& Information Security (ENISA) 'Baseline Security Recommendations for IoT', and 'Good Practices for Security of Internet of Things in the context of Smart Manufacturing'.
} 


\subsection{Deployment Scenarios}

Deployments for IoT devices that are used in the context of emergency services are typically deployed in three configurations:

- First, devices communicate with the ESINet via some service provider. This deployment model is, for example, found in roadside assistence solutions where the service provider offers a range of other functions besides emergency services support.

- Second, devices are connected to the cloud-based IoT service via some form of gateway. The use of a gateway is typically needed when devices use short-range radio technologies, such as IEEE 802.15.4 or Bluetooth Mesh. The gateway ensures that the exotic IoT radio technologies are integrated into the rest of the network infrastructure. Sometimes the gateway also offers application layer protocol translation. A smoke detector connected to a multi-hop mesh network using IEEE 802.15.4 is an example for such deployment.

- Third, IoT devices may be connected directly to the Internet using classical cellular technology or some of the recently developed low power wide area networking technologies, such as NB-IoT. A sensor built into a bridge is an example for this deployment.

Figure 7.1 shows these deployments graphically. A more detailed discussion about different IoT deployment models can be found in [113].

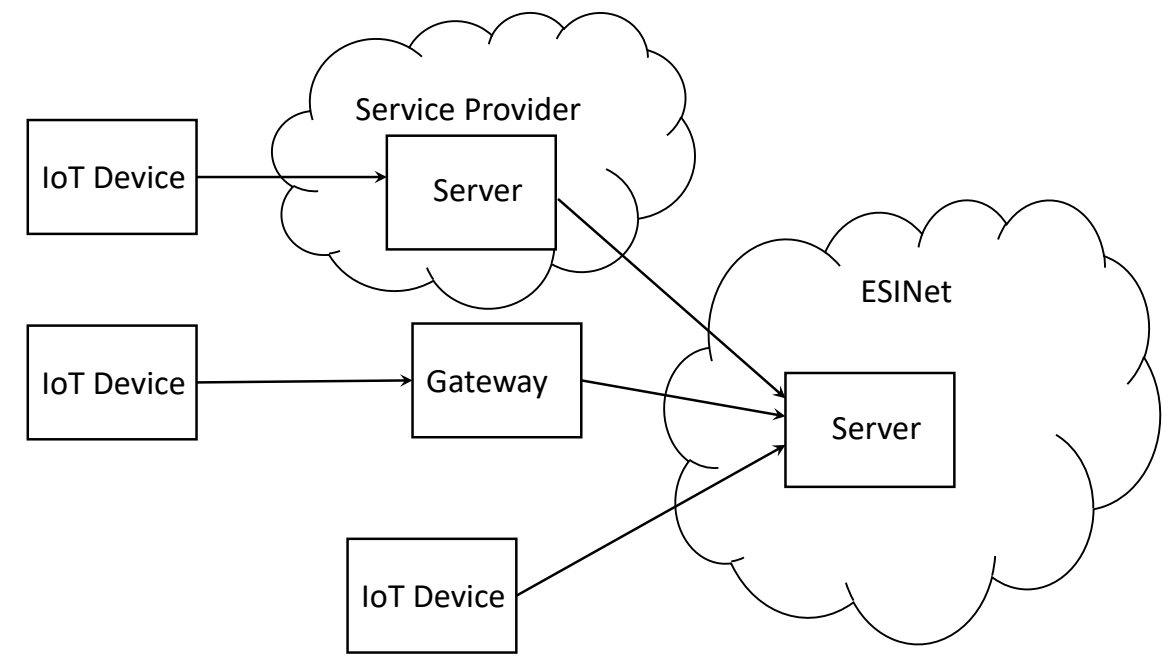

Figure 7.1.: IoT Deployment Scenarios.

In rare cases IoT devices directly interact with each other. 


\subsection{Security and Privacy Threats}

\subsubsection{Categories of Attacks}

The common approach to designing a security solution is to conduct a threat analysis first. Broadly, attacks can be categorized (as depicted in Fig. 7.2) into (i) network (or communication) attacks, (ii) software attacks, and (iii) hardware attacks.

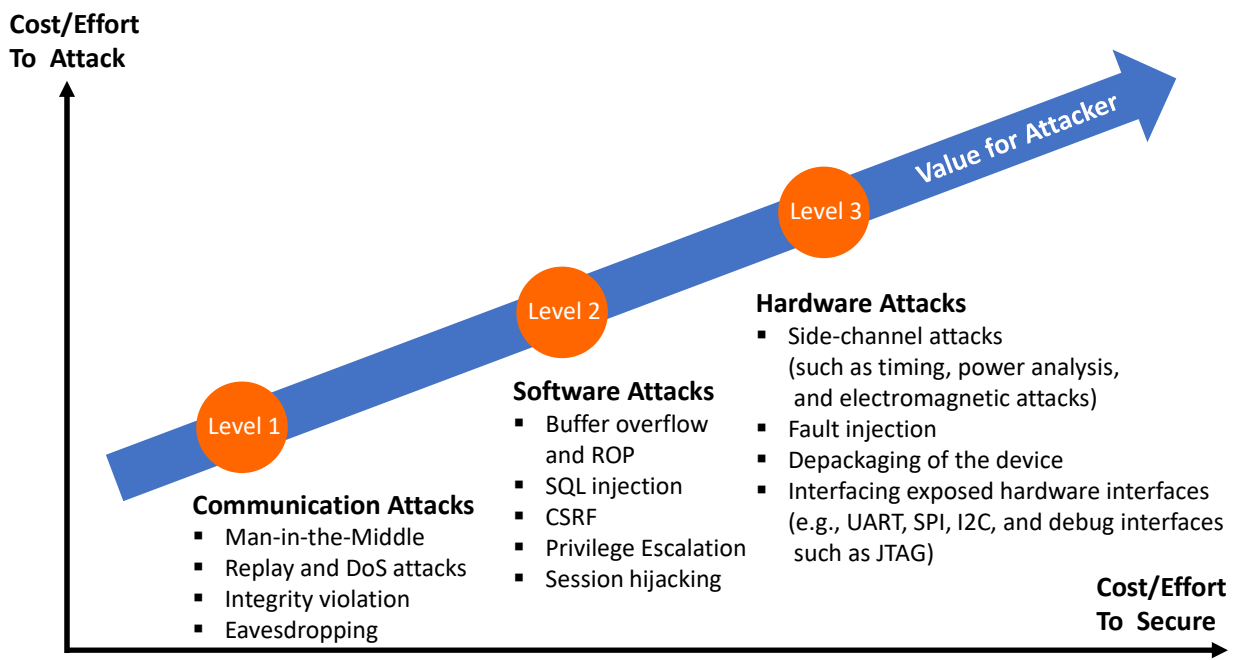

Figure 7.2.: Categories of Threats.

Historically, the IETF focused on countering network attacks, and ignored software and hardware attacks. For this reason, the main threat model, as described in RFC 3552 [114], is a classical network attacker who can carry out active and passive attacks against the communication interaction. Only recently, the IETF started tackling some security concerns beyond network attacks, with the work on attestation, trusted execution environments, and firmware updates (see Section 7.5.7). 
Privacy-related threats were added later with RFC 6973 [115] and are now being considered in the design of protocols throughout the IETF, including IoT protocols. In some scenarios there is an overlap of privacy and security threats (for example, in a surveillance scenario), and there are some privacy-specific threats, such as data minimization and user participation. The threat of pervasive monitoring, as revealed by Snowden, certainly had a huge impact on recognizing the importance of privacy in the design of Internet protocols, as documented by the IETF community in RFC 7258 [116].

\subsubsection{Threat Modelling}

In considering which assets have to be protected, in many cases it is fairly obvious what the threats are, even for someone who is less familiar with security. Unfortunately, threat analysis is a process that is iterative and creative because product design decisions can create new threats and the deployment environment may also change over time. As such, it is often necessary to examine the threats not only once, at the beginning of the product development process, but also to adjust the list of threats during the product lifecycle.

The following are three examples that illustrate the creative nature of the threat analysis activity:

- Consider an emergency services product that is sold to consumers and deployed in their home. Often these products are considered physically secure because a thief breaking into someone's home is probably less interested in tampering with a smoke detector. However, if the same customer sometimes rents his home to strangers, via services like Airbnb, the threat model has to be re-evaluated. This case illustrates a potential change in the deployment environment. Accurate threat modelling requires a good understanding of how IoT devices are used and other changes in the threat landscape over the lifetime of the device.

- Consider another emergency services product that uses state-of-the-art crypto with a rocksolid security protocol to authenticate and protect a firmware update mechanism. Imagine further that the engineers of this product decide to deploy the same symmetric key, a class key, on all devices of the same product family. In this scenario, an attacker only has to buy and analyze a single device - for example, by conducting a (novel) side channel analysis - to obtain the class key and ship a firmware update to all devices in the same product family. In the worst case, the attacker can deliver the firmware updates over the Internet without having to be in close proximity to the devices. This example shows how the changing capabilities of attackers can change the threats posed. The people conducting the threat modelling must be aware of the attackers' techniques and their level of sophistication. 
- Consider a final example where the threat analysis addresses all assets the company selling the product cares about. Later, it turns out that the product is vulnerable to a reflection attack, which was not considered in the threat analysis because the computing resources of third parties (the victims of the attack) were not included in the asset list. Those involved in threat modelling have to be aware of the type of attacks encountered in specific industries today.

\subsection{Overview of ENISA Guidelines}

Once an initial set of threats is collected, it is time to think about which of those threats should be dealt with, and how. Although a company may decide not to offer a technical solution to all security threats, it is useful to consult industry guidelines, such as the ENISA guidelines [112], which can help to make reasonable decisions. While many security requirements relate to the software and hardware implementation of a product, such as the boot process and hardware crypto, there are also many protocol-specific elements that are important security considerations.

Here is a list of the most important groups of security considerations:

1. Authentication and communication security

2. Object security

3. Authorization and access control

4. Key management

5. State-of-the-art crypto

6. Restrictive communication

7. Firmware and software updates

Many of the requirements that fall into these seven groups can also be found in other best current practice guides. In fact, there is an astonishing overlap among the guidelines produced by governments, industry groups, security experts, and researchers. This gives us confidence that these guidelines indeed represent a consensus of sorts among security experts.

Interestingly, the seven groups listed above can be mapped loosely to areas of work in the IETF. In the next section a high-level overview of the ongoing standardization work is provided. 


\subsection{IETF IoT Security Standardization}

The network security mechanisms surveyed aim to be applicable to all communication patterns described in RFC 7452 [113]. The deployment scenarios illustrated in Section 7.2 are most relevant in today's deployments.

\subsubsection{Authentication and Communication Security}

The IETF work on offering communication security for IoT devices resulted in the consolidation of Transport Layer Security (TLS) and Datagram Transport Layer Security (DTLS). RFC 7925 [117] defines profiles of TLS and DTLS 1.2 for use in IoT environments with separate guidance for three types of credentials supported by TLS/DTLS, namely pre-shared secrets, raw public keys, and certificates. Password-based techniques, which have also been defined for TLS/DTLS, are not discussed, because the industry is, in general, trying hard to move away from password-based authentication. Since the publication of RFC 7925 in 2016, more and more embedded TLS/DTLS stacks that support the recommendations in this specification are available for developers.

Over the last few years, the IETF has been busy standardizing the new version of TLS, in the form of version 1.3, inspired by the needs of the web developer community, which wanted to reduce the round-trip latency of the handshaking protocols. TLS required two roundtrips before application data could be exchanged securely. The standardization process for TLS 1.3, now published in RFC 8446 [118], was started in April 2014 and completed in August 2018. The list of changes and new features is long, which has contributed to the longer standardization process. The reviews by a large number of participants, early implementation and interoperability efforts, deployment investigations, and the use of formal methods have contributed to the longer standardization process, but these contributions significantly improved the quality of the specification.

The new features of TLS 1.3 are:

- A reduced number of roundtrips. The 0-RTT exchange enables transmitting application data with the first message (at the loss of replay protection, which has to be provided by the application layer). With the regular exchange, a client can send application data after the first roundtrip. To convey the necessary information, the client has to add extra information into the ClientHello message. The assumption is that the client will know the server, or at least maintain some information about the server, and that configuration information, such as algorithms, rarely changes. 
- The three Pre-Shared Key (PSK)-based modes (PSK-based authentication, session resumption, and session resumption without server-side state) have been merged into a single mode. Password-based authentication, which was available in TLS/DTLS 1.2 with SRP [119] and J-PAKE [120], was removed.

- Encryption of handshake information is made as early as possible. While this makes network monitoring and debugging more complex, it also increases privacy protection. The ability to add padding to messages was also added to make traffic analysis more difficult.

- A modified ciphersuite concept, in which the negotiation of the algorithms has been untangled from the authentication and key exchange. The ciphersuite list was thereby also cleaned up.

- Increased use of perfect forward secrecy, which requires a Diffie-Hellman exchange.

- Removed key transport, a decision which was met with considerable scepticism by enterprise network operators and firewall vendors since it requires more sophisticated interception techniques to perform deep packet inspection.

- Use of authenticated encryption with additional data as the go-to-choice for symmetric algorithms.

- Removed custom Diffie-Hellman groups and compression.

- Redefined renegotiation with the introduction of a post-handshake authentication mechanism.

- Added solid version negotiation.

- The key derivation algorithm now makes use of the HMAC-based Extract-and-Expand Key Derivation Function (HKDF) construct defined in RFC 5869 [121] and a more structured key hierarchy, which enables researchers to better analyze the handshake using formal methods because of the various keys are independent. From an implementation point of view, this change led to a larger code size compared to earlier TLS versions.

With this long list of features it was interesting to get more data about the impact on code size, message overhead, and the number of cryptographic operations. With a TLS 1.3 stack implemented on top of the Mbed TLS library an early conclusion can be drawn that TLS 1.3 provides an improvement for IoT deployments in terms of security, privacy and performance at roughly the same code size and with the same RAM requirements.

The work on DTLS 1.3 [122] was started with a slight delay and is currently being finalized. DTLS aims to provide communication security for connectionless transport protocols. This allows developers to secure protocols that are primarily used over the User Datagram Protocol (UDP), such as the Constrained Application Protocol (CoAP). The design of DTLS 1.3 reuses TLS 1.3 as much as possible. There are, however, several aspects that are worth highlighting: 
- DTLS requires an enhancement for denial of service protection. This is accomplished with a dedicated message in earlier versions of DTLS, namely the HelloVerifyRequest handshake message. In DTLS 1.3, this message has been replaced by the HelloRetryRequest message, which was already introduced in TLS 1.3.

- The record layer encoding has been optimized. The version number and type fields have been removed, the length of the epoch and sequence number fields have been shortened, and the header uses a variable length encoding. This lowers the over-the-wire overhead.

- The retransmission mechanism has been redefined. Prior to DTLS 1.3, the retransmission granularity was at the level of an entire flight (in other words, retransmission required a series of messages). In DTLS 1.3, an explicit ACK message has been defined to enable acknowledgements of handshake messages explicitly.

- DTLS 1.3 introduces a connection ID concept, which enables lookup of the security association of an incoming record based on a newly introduced identifier carried in the record layer header rather than using the source IP address and source port. The connection ID concept is particularly useful in an IoT context where devices sleep for extended time periods and likely run into the problem of an expired state with network address translators. In light of the popularity of this extension, it has also been backported to DTLS 1.2 [123], although with weaker privacy characteristics because DTLS 1.2 does not support post-handshake messages. Maintaining the same level of privacy while introducing this new feature was important in the design, and also led to the introduction of a sequence number encryption scheme for DTLS 1.3. This extension enables the use of an established security context for significantly longer without the need to rerun full or resumption handshakes, and results in a significant performance improvement.

We expect TLS and DTLS 1.3 to find widespread usage on the Internet and in IoT deployments.

When security requirements include end-to-end communication security, a reasonable question is what the 'endpoints' of this exchange are. In some cases, it may be sufficient to offer security at the link layer since the two ends are topologically close to each other and sometimes a more complex communication path is utilized. A properly described system architecture will give insight into what the participating entities are.

Here is a typical example of an IoT device in an emergency services context that communicates with support infrastructure provided by the manufacturer. The IoT device uses a radio technology to connect to the Internet; for example, to a Wi-Fi network. Once it connects to the Internet, it contacts a device management server. The device management server is responsible for configuring the device, and for managing software updates. The entire lifecycle of the IoT device is managed by the device management server. Depending on the deployment preferences this device management server may also be used to retrieve 
sensor values and to triggering actuators, if there are any. If we want to secure communication end-to-end, security protection has to start and end at the two endpoints, namely at the IoT device and the device management server. TLS and/or DTLS would be a fine choice for providing end-to-end security.

In addition to the TLS/DTLS, the IETF now offers another communication security solution called 'Object Security for Constrained RESTful Environments' (OSCORE [124]). The development of OSCORE was initially started as an application layer security solution protecting CoAP messages end-to-end. Later, it was extended to work also when CoAP traffic is mapped to HTTP because end-to-end communication in IoT deployments rarely uses CoAP alone. Since the SIP protocol is often used for emergency services OSCORE is not a good choice since OSCORE does not support SIP and SIP itself is not a RESTful protocol.

For those cases where CoAP is used instead of SIP, and DTLS/TLS is considered insufficient, OSCORE may be a possible candidate for a security protocol. OSCORE re-uses COSE [125] for protecting CoAP messages, or more precisely some CoAP headers and the CoAP payload. The reason it offers it cannot protect the entire CoAP message is that the CoAP also defines intermediaries, so-called CoAP proxies, and those proxies need to inspect part of the message. CoAP defines the interaction between CoAP and DTLS slightly differently to how HTTP did. In CoAP, DTLS is terminated at the proxy while in HTTP a TLS exchange is transparently passed through (unless it is a reverse proxy). Note that in context of OSCORE, these proxies can also be special CoAP-to-HTTP proxies as long as they do not change the nature of the REST API interaction (and for the CoAP-to-HTTP mapping guidance described in [126]).

Since the OSCORE specification does not define a key management mechanism, the ACE-OAuth framework [127] is re-used to facilitate that functionality. The design follows the use of DTLS/TLS between the ACE-OAuth client and the resource server whereby the authorization server issues a proof-of-possession token along with the key to the client. The client then uses that key to derive keys for OSCORE, and then secures the communication with the resource server using OSCORE. This solution [128] is now being standardization in the ACE working group.

\subsubsection{Object Security}

Communication security protocols typically protect the exchanges of parties over a longer period of time. These security protocols, as can be seen with TLS, often operate in various stages where computationally-heavy tasks are performed infrequently as part of a hand- 
shake, while application data protection is accomplished with symmetric keys derived during the handshake. We described those communication security protocols in Section 7.5.1.

In this section we focus on security technologies that are tailored toward one-shot payloads. For example, consider a scenario where firmware images are distributed by the device management server and must be secured end-to-end, from the developer to the IoT device. Since the firmware image may be stored on servers for an extended period of time and must be self-contained, a different security mechanism, such as COSE [125], is more appropriate, and we cover it next.

In computer science, we like to come up with encoding and serialization formats, and the most recent trend is the Concise Binary Object Representation (CBOR) [125], which was designed with a small code and message size in mind. To protect data that is encoded in CBOR, a new signing and encryption format, which is conceptually similar to JavaScript Object Signing and Encryption (JOSE), the signing and encryption format for JSON encoded data, had to be developed. (The functionality for JOSE is spread over different specifications; the most important documents are JSON Web Encryption (JWE) [129] and JSON Web Signature (JWS) [130]). JSON and JOSE was the way to encode data before CBOR and COSE came along. COSE can be seen as a set of building blocks that applications use in the way they find useful (with an eye on code size). A device implementing a firmware update solution may, for example, rely on asymmetric crypto and would, therefore, implement the signature verification capability offered by COSE and none of the other security services that use symmetric key crypto.

COSE offers the following security services:

- Digital signatures

- Counter signatures

- Message Authentication Code (MAC)

- Encryption

- Rudimentary key distribution methods

Most security services are offered in two versions, depending on whether one or multiple entities create or receive them. For example, the COSE_Sign1 signature structure is used when only one signature will be placed on a message, whereas COSE_Sign allows for one or more signatures to be applied. COSE_Sign1 and COSE_Sign are different structures and cannot be converted from one to the other.

The standardization work on COSE was finished 2017 and the RFC was published mid2017; therefore the availability of embedded COSE libraries is limited today. libcose and 
cose-c are two early implementations, released mid-2018, tailored for use in the microcontroller environment.

Since CBOR is a good fit for securing data when the goal is to protect one-shot messages, the use of COSE is one option for protecting firmware updates, or more precisely, for signing metadata about the firmware and, optionally, encrypting the firmware image. COSE can also protect access tokens used with the ACE-OAuth framework. Both examples are described in more detail in subsequent sections.

Since emergency services standards do not encode data in JSON or CBOR there are five approaches for securing such data:

1. New specifications are defined that define a new serialization format. For example, it is possible to encode a PIDF-LO in JSON or in CBOR. This approach allows the use of JOSE and COSE, respectively.

2. No changes to the existing specifications are made and data is treated as an uninterpreted byte sequence. This specifically allows the use of signatures over detached data.

3. Available security mechanisms suitable for use with the existing serialization formats are used. For example, in case of XML this would be XML digital signatures and XML encryption. Other data can be protected using the Cryptographic Message Syntax (CMS). CMS uses ASN.1 under the hood, which is also used in X.509-based certificates.

4. A compression technique is used on data that is encoded in XML before it is signed and/or encrypted. Such approach may only require a new media-type to be used, or a new media type to be registered with IANA.

5. Communication security is provided by TLS/DTLS where possible with object security only for selected parts, such as firmware updates.

\subsubsection{Authorization and Access Control}

Securing communication interactions is an important step to mitigate basic attacks. Many IoT devices used for emergency services, such as vehicles eCall systems as well as sensors, may need to be accessed by technicians and even end users. This interaction raises a number of authorization questions, including

- How can access to the device be secured, without inadvertently allowing unauthorized access?

- Is it possible to support different users or groups of users?

- Can the access rights of one user be set differently from other users? 
- Can access control policies be managed centrally?

- Does the solution scale for a greater number of devices?

- How can strong authentication mechanisms be utilized?

The IETF Authentication and Authorization for Constrained Environments (ACE) working group has developed a solution to answer the above-listed questions, based on the widely used OAuth 2.0 protocol [99], and brings fine-grained authorization to the IoT world. This body of work is called ACE-OAuth [127] and the specifications are already further along with interoperability tests taking place. While OAuth 2.0 was designed with a wide range of use cases in mind - such as the web, native apps on smart phones, tablets and desktop computers, browser-based apps, and even devices with a limited user interface, such as TVs, picture frames, and game consoles - a few enhancements had to be made to tailor OAuth to the constrained IoT environment.

First, let us briefly summarize how ACE-OAuth is expected to work at a high-level. The OAuth system consists of four main entities, namely the client, the resource server, the authorization server, and the resource owner. The client wants to access a protected resource at a resource server. In an example of a vehicle-based emergency services solution, the client could be software running on the tablet of a technician, and the resource server is software running on a microcontroller inside the vehicle. The authorization server is responsible for issuing tokens that allow the client to access the emergency services system in the vehicle such that the authorization policies stored at the authorization server are met. These authorization policies may be created by the car manufacturer (acting as a resource owner) or phrased differently the resource owner decides about who gets access to the protected resource.

The token, which is issued by the authorization server and consumed by the resource server, is called the access token. The OAuth working group standardized a token format, called JSON Web Token (JWT) [98], which encodes claims in JSON, and the token itself is protected using the mechanisms developed in the IETF JOSE working group. Since the OAuth 2.0 specification does not mandate a token format, it is possible to design and use a token format that works best in each environment. In the case of ACE-OAuth, a counterpart to the JWT was developed with the CBOR Web Token (CWT) [131], which uses CBOR instead of JSON, and COSE and instead of JOSE. The result is a smaller token size.

Access tokens used in OAuth 2.0-based deployments are mostly bearer tokens. Proof-ofpossession (PoP) tokens were introduced later $[132,133]$, and in the IoT environment it is possible to use PoP tokens from the beginning. The key characteristic of a PoP token is that the token itself is associated with a symmetric or asymmetric key, and the entity presenting the token must demonstrate possession of the key. When a public key is associated with the 
PoP token, this means that the client must use the private key along with a digital signature when demanding access to a protected resource on a resource server. An attacker, therefore, needs to steal the key associated with the token in addition to the token itself. The use of PoP tokens requires an enhancement to the original OAuth 2.0 specification because extra information about the keys to be bound to the tokens must be passed around.

The second enhancement to the OAuth 2.0 specification accommodates for the different protocols being used in the IoT environment. While HTTP is used for IoT communication, the Constrained Application Protocol (CoAP) and the Message Queuing Telemetry Transport (MQTT) protocol are also popular. OAuth 2.0 has not been developed for use with these protocols, and the necessary extensions are defined in the IETF ACE working group.

Concurrently with the work on ACE-OAuth, work on an Entity Attestation Token (EAT) format [134], which introduces attestation-specific claims for use in the CWT structure, has been contributed to the IETF. The promise is to enable IoT devices to communicate information about their hardware and software characteristics to other communication parties. This work is still at an early stage, and the ambition is to create a working group. These EAT tokens could provide information about the manufacturer, used hardware security features, and hashes computed over the bootloader and firmware code of the device to a communication party, opening up additional decision-making possibilities. For emergency services applications the extra security properties provided by this attestation information could be highly valuable.

\subsubsection{Key Management}

IoT devices need several keys to enable lifecycle management and remote management. As explained in the Internet Protocol for Smart Objects (IPSO) Alliance (now OMA SpecWorks) publication on credential management for IoT devices [135], a common security assumption is that IoT devices have been provisioned with at least one long-term credential during manufacturing. This long-term credential is then used to provision further keys to the device in a process called bootstrapping, commissioning, onboarding, or enrollment. This new terminology hides the fact that the developed protocols provide two main steps:

1. The device authenticates itself to another party and vice versa using a pre-provisioned credential. In many cases, this credential is provided during manufacturing. For example, a device may be provisioned with a unique device certificate along with a private key. This credential is then used to authenticate the device to the other party in a communication interaction. To offer mutual authentication, the device must also be provisioned with one or multiple trust anchors. There is, however, one important exception where devices start 
without having credentials provisioned, and they use an out-of-band mechanism to obtain security guarantees. Bluetooth Low Energy, for example, uses this approach where in proximity, a PIN entry, is used as a security guarantee to skip the provisioning step.

2. A protocol exchange is required to convey or derive new credentials. These new keys, often called operational credentials, are used to secure the communication of the device, including data and configuration exchanges.

Many vendors and standards developing organizations have designed protocols that provide this bootstrapping functionality. Some of these protocols are specified for use with a specific radio technology (as the Bluetooth Low Energy example shows) and others are radio technology agnostic (or at least to a large extend). Organizations and vendors that have developed these bootstrapping techniques include OMA SpecWorks (with the Lightweight Machine-to-Machine (LwM2M) protocol [136]), Open Connectivity Foundation (OCF), Thread Group, Intel (with their Secure Device Onboarding), IEEE (Device Provisioning Protocol), Alljoyn, WiSun, Zigbee, and Bluetooth (Mesh).

Unsurprisingly, the IETF has also developed a number of these protocols for use in IoT environments, as outlined in this survey [137]. Examples include ZeroTouch, ANIMA/BRSKI, Enrollment over Secure Transport (EST), EAP-NOOB, ACE-OAuth, Certificate Management Protocol (CMP), PANA, and EAP/AAA.

\subsubsection{State-of-the-Art Crypto}

Cryptography is a conservative business. Developing a new cryptographic algorithm, writing proofs, publishing papers, and standardizing the cryptography is not enough to make it see widespread adoption, or any adoption at all. Years of community-wide review is typically required, which makes cryptography quite costly in terms of development. An engineer given the task of developing software for an IoT product is therefore well served to rely on off-the-shelf crypto rather than going for the cutting edge. Resisting the urge to develop your own cryptographic algorithm is key.

With the work on the TLS 1.3 specification [118], the IETF TLS group cleaned up the ciphersuite list, and the recently published TLS 1.3 specification, therefore, contains a list of algorithms that can be considered a good choice. We use the crypto recommendations from the TLS 1.3 specification since the RFC has recently been published and it represents the consensus of the IETF community in terms of crypto protocols. While these algorithm recommendations are tailored to TLS 1.3, they are typically also applied to other protocol designs. The recommendations have been developed in cooperation with the Internet Research Task Force's (IRTF) Crypto Forum Research Group (CFRG). Here is what the spec 
suggests:

- For symmetric key cryptography, the industry has moved to authenticated encryption with additional data (AEAD) ciphers. AES-128-GCM-SHA256 is the 'must'-implement algorithm, and the same AEAD algorithm with a longer key size, namely AES-256GCM-SHA256, is a 'should'-implement. As a backup cipher, CHACHA20-POLY1305SHA256 has become popular and it is also a 'should'-implement.

- For digital signatures with certificates, the TLS $1.3 \mathrm{spec}$ indicates that RSA-PKCS\#1SHA256, RSA-PSS-RSAE-SHA256 and ECDSA-SECP256r1-SHA256 are mandatory to implement.

- For the key exchange, the NIST P-256r1 curve (secp256r1) is mandatory to implement. Implementations 'should' support key exchange with X25519, which is an alternative curve (Montgomery).

There are two caveats:

- First, IoT devices often implement the Counter with CBC-MAC (CCM) in hardware rather than the Galois/Counter Mode (GCM). The CCM mode of operation with AES has been put into most, if not all, IoT specifications as the preferred algorithm. To reduce the overhead caused by the message authentication code (MAC), CCM also supports a variant with a shortened MAC, referred to as CCM-8. CCM-8 is commonly used in IoT deployments.

- Second, most IoT deployments use Elliptic Curve Cryptography (ECC) rather than RSA. As such, the algorithm of choice for certificates today is ECDSA-SECP256r1-SHA256.

In the future, we may see convergence in the area of symmetric crypto algorithms used on IoT devices and on the general Internet with ChaCha20 and Poly1305. It is also worth noting that there have been standardization efforts around lightweight symmetric cryptography - for example, by NIST - but it is too early to say whether these algorithms will be useful beyond a niche market. Modern low-end IoT hardware is equipped with a True Random Number Generator (TRNG), very often supports SHA-256 and AES-128, and may support ECC-crypto. This is already a big improvement compared to the availability of even TRNGs in microcontrollers a few years ago. The use of a TRNG is essential for security protocols because almost all security protocols rely on a source of randomness, for example, for nonces and key generation. 


\subsubsection{Restricting Communication}

Most IoT devices are built to fulfil a specific purpose; typically to collect sensor input, process it, and act on the results. In edge or fog computing, even sensor fusion is often outsourced to a gateway, or it is delegated to the server-side infrastructure. Naturally, the communication interaction of such IoT devices is also limited, particularly since these devices have limited RAM, which prevents communication with multiple hosts concurrently.

The earlier work of the IETF Network Endpoint Assessment (NEA) working group aimed to develop an architecture and protocols to "assess the posture of endpoint devices for the purposes of monitoring compliance to an organization's posture policy and optionally restricting access until the endpoint has been updated to satisfy the posture requirements". At the time that NEA was standardized, the endpoints were mostly laptops and desktops in enterprise networks. It became very difficult to write policies that described the posture policy in an organization for all endpoints, given their varying communication interaction methods, and then to keep that policy up-to-date. In the end, the NEA standardization work had less security impact than expected.

With the much simpler communication interaction of IoT devices, the idea of restricting the communication of these devices surfaced again in the work on the Manufacturer Usage Description (MUD [138]). An organization interested in formulating a policy for what an IoT device is allowed to do faces the challenge that it requires some analysis work to determine which protocols a device is using, and with which hosts it is communicating on the Internet. Only the company developing the final product has complete information available about the communication behavior since it is in full control of developing (or selecting) the software stack.

Although IoT security recommendations highlight the need to remove unused services, network operators still inherently distrust the manufacturers of these devices. The current assumption seems to be that IoT devices will not be developed with security in mind. This lack of confidence in the ability to develop secure IoT devices has led network equipment manufacturers, network operators, and security start-ups to develop various schemes for "learning" whether an IoT device behaves correctly. While products on the market use some form of deep packet inspection and analysis of communication interactions by intermediaries somewhere in the access network, the standardized solution expects the equipment manufacturer to publish MUD files that offer information about the communication interaction of the device. These MUD files are communicated from the IoT device to the network using Dynamic Host Configuration Protocol (DHCP), or as part of the network authentication procedure via extensions in certificates. Equipment in the network then fetches these MUD files and uses the machine-readable description to create firewall policies auto- 
matically. For example, it is expected that a device that misbehaves due to an attack will be firewalled, or at least that alerts will be generated. A manufacturer has to update these MUD files for each product whenever the communication interaction changes; for example, when the device interacts with different servers or uses alternative communication protocols.

At the time of writing, the standardization work on MUD is largely completed and it remains to be seen whether manufacturers who previously did not care about implementing a firmware update solution and alike can now be convinced to publish MUD files about their products in a timely fashion. At the same time, many companies are trying hard to improve the security of endpoints with new hardware security mechanisms and with readily available open source code that takes most of the difficult security programming out of the hands of developers.

\subsubsection{Firmware and Software Updates}

Providing a firmware update solution for IoT devices is essential to dealing with bugs and the changing environment. While this seems obvious, we still see lots of devices in the market that do not enable updating their code. Best current practice guides not only demand the ability to update firmware, but also the ability to do so securely. In 2017, the IETF formed the Software Updates for Internet of Things (SUIT) working group to standardize an IoT firmware update solution. The initial starting point was to outline the architecture [139] and the metadata describing a firmware image along with its security mechanism [140]. The metadata contained in a data structure that is protected, at least against modifications, is called a manifest. IoT device management solutions, such as LwM2M [136], can then be used to deliver the manifest and the firmware image to IoT devices.

The manifest contains several elements to instruct an IoT device to install only firmware that comes from an authorized source, has not been modified, is (optionally) confidentiality protected, is suitable for the hardware, and meets various other conditions. One item of the IETF SUIT working group is an information model document, which explains the purpose and the semantics of the manifest elements.

The IETF SUIT aims to cover a wide range of use cases, not only because the IoT market is quite diverse in its deployment needs, but also because of the hardware being used. Since many IoT devices today contain multiple microcontrollers to perform different tasks, such as radio communication and application processing, the manifest also has to provide an indication of which microcontroller must be updated. Likewise, a single microcontroller may have software from different vendors, such as a Bluetooth software stack from the chip manufacturer and application code from a developer. Transferring large firmware images 
can be a challenge for low-power radio technologies, and some vendors, therefore, prefer to use differential updates or to update selected components instead. The manifest also has to cover these use cases.

Dealing with different versions of firmware over the lifetime of the device is another a requirement. These scenarios require some form of dependency mechanism and version management. The use of an encrypted firmware image is also becoming more popular because it reduces the attack surface since knowledge of the code running on an IoT device, even if it must be reverse engineered with tools like Radare, Binary Ninja, or IDA Pro, gives an attacker a lot of additional insight. Obtaining a firmware image is often the first task in an attack chain.

While the standardization process in the IETF SUIT group is still ongoing, there has been interest in reusing existing building blocks, such as CBOR and COSE (discussed previously in this article), to encode and protect firmware images, respectively. Even if vendors only ever update their own device in a siloed IoT deployment, it is still beneficial for them to rely on a standardized solution to give others confidence that the solution has been designed properly with input from a wide range of industry players, follows best current practices, and potentially uses off-the-shelf code. The reuse of code also helps to reduce the development effort since participants in the group intend to release a reference implementation as a byproduct of the standardization activities. Some of the participants have verified the ongoing specification work at IETF hackathons.

The IETF SUIT working group is somewhat related to the Trusted Execution Environment Provisioning (TEEP) working group since the goal in both cases is to update code on a device. The big difference between SUIT and TEEP is that TEEP focuses on hardware that uses Trusted Execution Environments (TEEs), such as Arm TrustZone or Intel SGX. A TEE is designed to provide a hardware-isolation mechanism to separate a regular operating system from security-sensitive application components, such as key storage. The goal is, therefore, to update code that runs inside these TEEs, the so-called trusted apps. Most of the hardware equipped with a TEE today - for example, gateway devices in IoT deployments is rather powerful compared to a constrained IoT device; however, with the recent addition of TrustZone technology to the Arm M-profile architecture, constrained microcontrollers are gaining trusted execution capabilities.

The TEEP working group is standardizing an application layer protocol, the Open Trust Protocol (OTrP [141]), which manages the interaction between a TEE and a server-side component, the so-called Trusted Application Manager (TAM), to query the TEE for installed trusted apps and to manage the lifecycle of these trusted apps. Trusted apps are only installed if the TAM has successfully attested the hardware and software functionality of the TEE. The design of OTrP is complicated by the interaction of the trusted app and 
code running on the regular operating system, the different ways of delivering software to higher-end devices, such as phones and tablets, and because devices may be equipped with multiple TEEs. The group agreed to look only at the use of public key cryptography in OTrP. The TEEP architecture specification [142] provides further details.

\subsection{Conclusion}

Designing a secure IoT product is hard, as press releases about hacked IoT products demonstrate. In the meanwhile, many guidelines for securing IoT devices have been published and we focused our attention to the 'Baseline Security Recommendations for IoT' document published by the EU Agency for Network \& Information Security (ENISA) but other guidelines make similar recommendations. In this survey we have looked at IETF standardized security solutions specifically designed for constrained IoT devices in the categories communication security, authorization, key management, cryptography, restricting communication, as well as firmware and software updates. These seven categories have been created based on the list of recommendations by ENISA.

We asked ourselves whether it is even possible to develop a reasonably security product based on the standardization work done by the Internet Engineering Task Force (IETF). As far as the scope of the IETF work goes, we believe this is possible. Standardization work has advanced to a point that there is typically no need to build home-grown solutions.

There are, however, challenges and gaps as well. At the time of writing some standardization work is not yet completed. In other cases, standardization work is ahead of implementations. More resources are needed to implement high-quality embedded libraries. Even a protocol that is theoretically secure is only secure in practice when implemented without relevant bugs. As such, do not ever write your own crypto implementation and use a well tested, widely used libraries instead since protocol implementation bugs and subtleties in specifications are common.

Since even well-tested security protocol implementations have bugs it is important to not underplay the importance of firmware and software updates. It is probably the number one security feature of a product.

In the context of security libraries it is also worth to point out that these libraries need to be developer-friendly and available for use with the large number of IoT operating systems. Today, developers often face a lot of pain when integrating security libraries into the IoT hardware of choice. 
For engineers who want to advance their development quickly we recommend starting with an IoT device management framework, such as LwM2M, rather than selecting individual building blocks and combining them to something meaningful. This approach has the advantage that a working system is built rather quickly. Configuration and optimization potential is still available with each of the IETF-developed security protocols, assuming an engineer has security know-how and is familiar with embedded development. 



\section{Chapter 8}

\section{Conclusion}

\subsection{Summary}

Emergency services support is one of the most valued functions of the telephony system. With the transition from circuit switched telephony to IP-based multimedia communication, many design decisions had to be re-evaluated. The desire for separation between application service providers and providers offering Internet connectivity allowed application service providers to expand their services to customers around the world. As a consequence, additional requirements for the global use of emergency services were placed on the design of an IP-based solution. This thesis describes the design of the IETF emergency services architecture that can be used by any application, including vehicles and Internet of Things devices. Due to the modular design various building blocks can also be used by other architectural variants and in transition deployments. Designing functionality without considering security and privacy is not a feasible approach anymore. Hence, security and privacy features have been the main focus throughout the design of the presented emergency services architecture.

The first few chapters provide an introduction, list requirements, and summarize related work.

Chapter 5 gives an introduction to IETF IP-based emergency services and illustrates how emergency call routing works. This architecture meets the requirements outlined in Chapter 2 and offers advantages over the alternative solutions presented in Chapter 3 due to its generic nature and global applicability. A comparison with alternative architectures is provided in Chapter 4. A core component of the IETF emergency services architecture is the ability to obtain routing information based on the location information and service URN provided. The LoST protocol provides this functionality. Details of the LoST protocol im- 
plementation are available in Annex A. The performance analysis shows promising results even on an off-the-shelf desktop PC: The minimal roundtrip time (RTT) of the LoST request/response is $39 \mathrm{msec}$ and the maximum is $856 \mathrm{msec}$. The median is $55 \mathrm{msec}$ and the mean is $72.17 \mathrm{msec}$. The first and third quartile is $48 \mathrm{msec}$ and $65 \mathrm{msec}$, respectively.

Security and privacy is a common concern with any Internet-based application, and a system that aims to save lives is quite naturally subject to more scrutiny. In Chapter 6 security is analyzed from a broad perspective, and threats that surfaced in discussions are captured. Luckily, most threats can be mitigated with the use of various security technologies. However, there is one threat of particular concern that relates to the ability to attack the limited resources of call takers and first responders. In the November 2011 issue of the Communications of the ACM magazine [143], we highlighted the problem of caller identity spoofing, as used in swatting. Identity spoofing, fake location, and SIM-less emergency calls remain a problem.

Chapter 7 enhances the classical emergency services concept to include Internet of Things devices like temperature sensors, burglar alarms, and chemical spill sensors. With the weak security features offered by today's IoT devices, this chapter provides a survey of security protocols made available by the IETF. The survey gives hope: developers can pick from range of standardized security technologies to make their IoT devices more robust against attacks.

The work on this IP-based emergency services architecture represents an example of security and privacy by design.

\subsection{Future Work}

A large body of standardization work has been finalized during the last few years; various product implementations are available and early deployments already exist. Still, there is much additional deployment work ahead, particularly since today's emergency services networks are disconnected islands. Although the increasing deployment experience will lead to adjustments to the envisioned architecture, new communication protocols, such as WebRTC [43], will introduce additional challenges because of use of non-SIP-based protocols.

Accomplishing widespread deployment of the envisioned IP-based architecture, as described in this thesis, requires more than just a technical solution documented in specifications. More than any other environment, the emergency services field is a complex ecosystem impacted by the business models of various actors (such as network operators, applica- 
tion service providers, emergency service equipment manufacturers), regulatory mandates, and the directions taken by individual countries.

The ability to obtain highly accurate location information has substantially increased with the existence of interoperable standards, the availability of implementations in products, and the rise of smartphones. However, this has not yet led to better location support for emergency services in many countries. Regulatory mandates governing how network operators deploy location servers for use with emergency services vary substantially throughout the world. In Europe, locations provided to emergency services are either civic addresses for land-line callers or locations based on Cell-ID for mobile phone users. The quality of CellID-based positioning varies substantially, and can, in urban areas, lead to a poor accuracy of several kilometers [144], [145]. Providing proper incentives for fixed and mobile operators and for enterprises to deploy location technology has been a struggle for a long time, and this situation has not changed during the period of writing.

Data collection by all stakeholders would be essential in allowing researchers to gain insight into the current state of emergency services deployments. While a certain amount of data is already collected today, it is either insufficient or not available to researchers for analysis. Without deployment statistics, researchers have little ability to diagnose problems, evaluate solutions with regard to effectiveness, or develop alternative technologies. For example, consider the following questions: How much can emergency services operation, and particularly dispatch, be improved with better location accuracy? At what point does the improved location lead to little improvement in dispatch times? These questions are unanswered today since there is no consistent data collection about the actual location of the emergency caller (whoes location was reported), or the time it took to reach the person of help.

Location is, however, not the only area where work is needed. Concerns about security and privacy are common with emergency services, and of course with the Internet in general. Unfortunately, the concerns are often unspecific and therefore hard to discuss and to dispel. Untangling the wide range of possible concerns is the first step in making progress. In Chapter 6, security is approached holistically.

There are three areas of security where further work is required.

- First, many of the existing VoIP and application service offerings provide only a limited form of identity proofing, and their user authentication technology is often weak as well. Without the ability to link a specific call to a real-world person, it is difficult to punish misbehavior. There is currently a larger body of ongoing work to develop and deploy better authentication and identity services on the Internet.

- Second, emergency services networks are IP-based networks and, if not operated cor- 
rectly, and updated frequently, they quickly become vulnerable to various forms of attacks.

- Finally, companies developing Internet of Things devices need to be incentivized to follow the current best practice guidelines. Hacked consumer devices already present a danger for the wider Internet via distributed denial of service attacks. When these devices are used for emergency services, the consequences are even more severe.

In most countries, telecommunications regulators at all levels, such as the Federal Communication Commission (FCC), the European Commission, and the UK Office of Communications (OFCOM) have a strong influence on how emergency services are provided in individual countries, who pays for them, and what obligations the various parties have. Regulation is evolving, and the requirements for emergency services support and for providing automatic location information vary.

The separation between Internet access and application providers is one of the most important differences from existing circuit switched telephone networks. A side effect of this separation is the increased speed of innovation at the application layer; the number of new communication mechanisms is thus steadily increasing. Many emergency service organizations have recognized this trend and advocate for the inclusion of new communication mechanisms, including video, real-time text, and instant messaging, to offer improved emergency calling support for citizens. Again, this requires regulators to re-think the distribution of responsibilities, funding and liability.

For many communication systems in use today, however, it is difficult to trace malicious activities back to the offender. This is not a completely new problem, as pay phones and prepaid cell phones have long offered mischief-makers the opportunity to place hoax calls, but weak user registration procedures, the lack of deployed end-to-end identity mechanisms, and the ease of providing fake location information increase the attack surface at PSAPs. Attackers also have become more sophisticated over time, and use botnets to generate a large volume of automated emergency calls to exhaust PSAP resources, including call takers and first responders.

The appendix complements the dissertation with a detailed description of the LoST implementation. It is too detailed to be included in the main part of the thesis but the content is nevertheless an important result of the dissertation. 


\section{Chapter A}

\section{LoST Software}

This annex contains a description of the software architecture of the prototype LoST client and server implemention developed to verify correctness and completeness of the standardization work done in the IETF. Typically, LoST will be integrated into a VoIP client and VoIP proxy/gateway. This write-up focuses on the LoST software components and neglects the software integration into a SIP stack.

The description for compiling, configuring and runnning the software focuses on Windows. The software can be downloaded from http://ecrit. sourceforge.net.

\section{A.1. LoST Client}

The LoST client is written in $\mathrm{C}$ and makes use of the libxml 2 third party library for parsing XML structures. For use on Windows-based systems the getopt library has to be included as well. For testing the LoST client a command line interface (CLI) is provided, which accepts the parameters shown in Listing A.1. These command line parameters correspond to the different features provided by the LoST protocol.

Listing A.1: LoST Client Command Line Interface Options

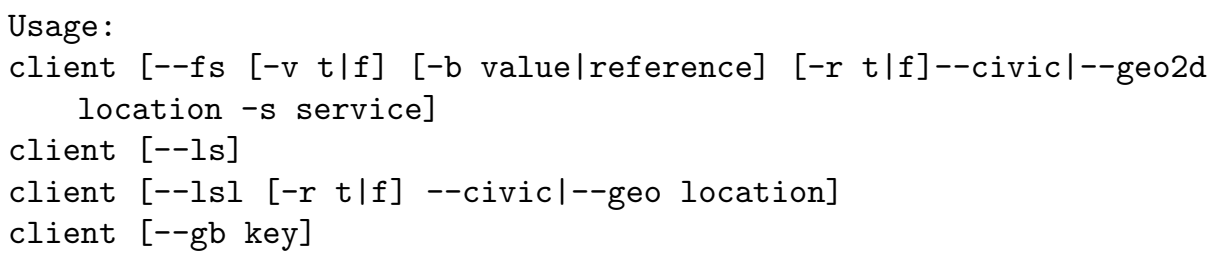

Main options: 


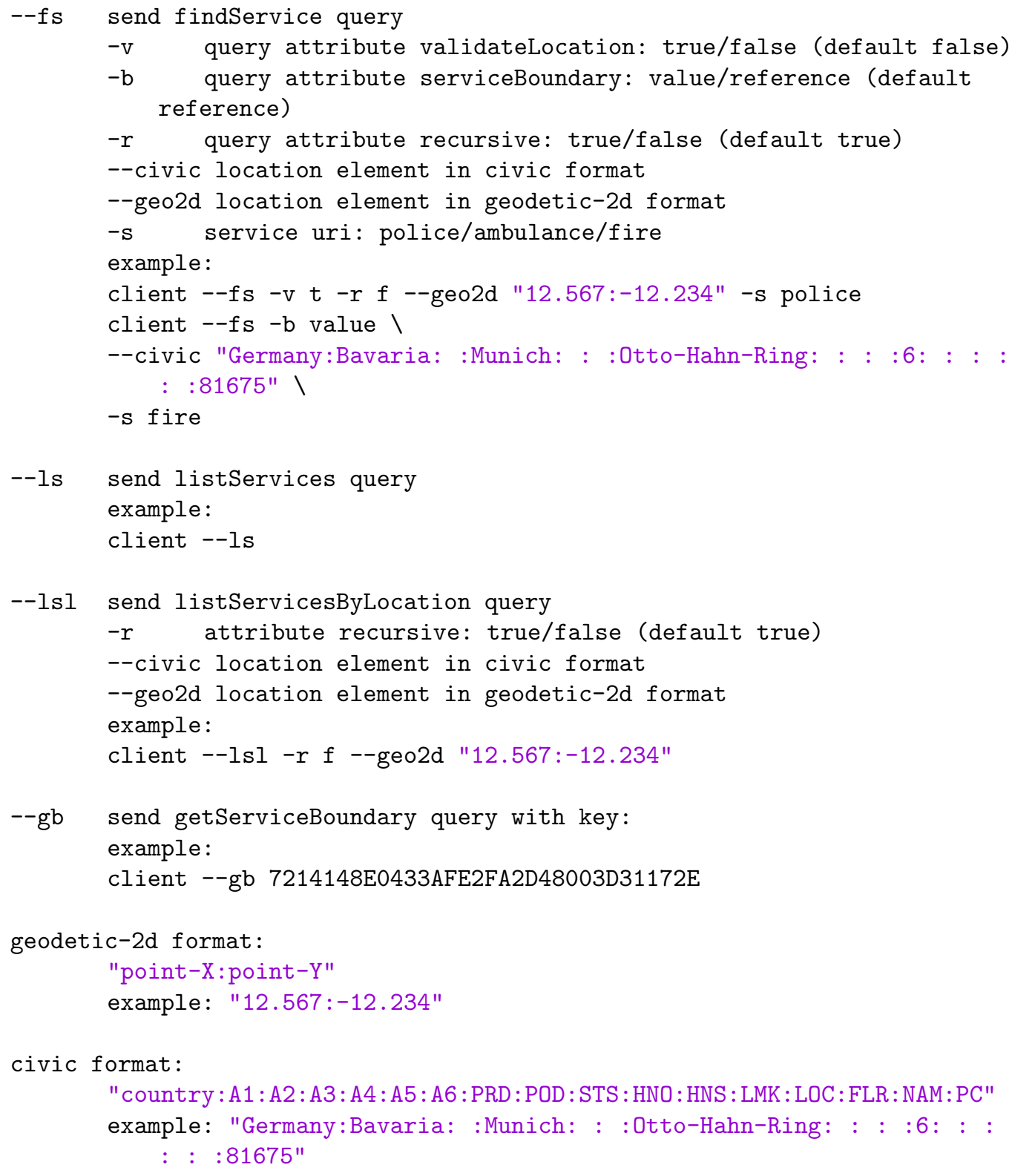

For the performance evaluation the findService query with geodetic location information is most important; the listServices, listServicesByLocation, and the getServiceBoundary queries play a secondary role. The LoST client implementation only supports a single geodetic location shape of the geo2d location profile, namely the Point. The Polygon, Circle, Ellipse, and ArcBand structures are not supported. For the purpose of this evaluation 
this is not a significant constraint given that GPS-enabled devices will mainly use the Point location shape (or a circular area to express uncertainty about a point). More complex location shapes are typically the result of network-based location determination techniques, which are not used in this performance evaluation.

An example LoST client configuration is shown in Listing A.2. The LoST client needs to be configured with the IP address and port number of the LoST server. Additionally, it needs to know the URL path, which is constructed in this example configuration using the Eclipse project name and the servlet name.

Listing A.2: LoST Client Configuration Example

serverLoST $=127 \cdot 0 \cdot 0.1$

page $=/$ LoSTAppRoot/StartServlet

port $=8080$

An example findService query with geodetic location information is shown in Listing A.3. The query instructs the LoST Server to use the police service URN to request a service boundary by reference (rather than by value) and an interative query (rather than a recursive query). The GPS coordinates for a location in Manhatten, New York are expressed as a latitude:longitude pair. This location refers to the following civic address determined via reverse geocoding: Sidewalk Clock at 200 5th Avenue, 5th Avenue, New York, NY 10035, United States of America.

Listing A.3: LoST Client CLI Query with findService Query Example

./client --fs -b reference -r f --geo2d

"-81.1562038362026:40.4049754631706" -s police

The LoST client implementation is separated into several files, namely

findService findService. $h$ and findService.c implement the findService query functionality getServiceBoundary getServiceBoundary.h and getServiceBoundary.c implement the getServiceBoundary query functionality

listServices listServices.h and listServices.c implement the listServices query functionality

listServicesByLocation listServicesByLocation.h and listServicesByLocation.c implement the listServicesByLocation query functionality

location location.h defines the data structure for civic and geodetic location information. location.c defines function to populate the data structure.

get_config get_config.h and get_config.c implement the functionality to read the configu- 
ration file.

cl_client cl_client.h defines constants for CLI usage of the LoST client and cl_client.c implements the CLI parameter parsing. cl_client.c also calls functions for LoST functions and contains code for parsing civic information provided via the CLI.

http_connection http_connection.h and http_connection.c implement the HTTP request/response interaction. Because of the different socket interfaces on Windows and Linux-based systems the implementation varies between these two operating systems.

common common. $h$ defines constants for debug, XML parameters, and emergency service URIs.

client client.c is contains the main() function of the LoST client CLI program.

The LoST client reads the configuration file, parses the CLI parameters and invokes the functions for building the respective LoST request. Then, the HTTP request is made to the LoST server. Note that the prototype uses regular HTTP for communicating with the LoST server. Then, the LoST client waits for the response from the LoST server, and prints the response message. Finally, the HTTP connection is terminated and after releasing resources the LoST client program is terminated.

To build the code for the LoST client on Windows it is easiest to use Microsoft Visual Studio and use the provided project files. When building the code from scratch (via Microsoft Visual Studio) import all source and header files and use the NuGet package manager to include the libxml2-vc140-static-32_64 and the libapr-iconv libraries along with any dependencies. Additionally, the libraries wsock32.lib and libxml2.lib have to be added as an additional dependency in the linker configuration. Note that the lost_client.conf must be in the same directory from which you run the LoST client executable.

\section{A.2. LoST Server}

The LoST server implementation is more complex than the client-side because it has to connect to a database to store service boundaries. The LoST server is implemented in Java and to run the Java application a Tomcat server is used. As a database PostgreSQL was selected because it supports geospatial functionality via the PostGIS extension. For software development Eclipse was used as an open source integrated development environment. To execute the LoST server the Java SE Development Kit has to be installed and for development the Java Development Kit (JDK). The implementation relies on the third party XML library JDOM2, which provides a Java-based implementation for accessing, manipulating, and outputting XML data. JDOMs SAXBuilder class can also be used as an XML parser. 
On Windows it is easiest to install PostgreSQL as part of the EnterpriseDB software from https://www.enterprisedb.com, which is a pre-bundled version of the necessary software to run PostgreSQL. The "StackBuilder" utility is then used to install the PostGIS add-on. Configuring and optimizing a database system can be a challenge but PostgreSQL offers a convient tool called PgAdmin 4, which offers a graphical interface to connect to a database, to manipulate tables and views, and to execute queries among many other functions. Access to the PostgreSQL database via the Java app is accomplished via Java Database Connectivity (JDBC) for which a driver has to be downloaded from https://jdbc.postgresql.org/download.html and added to the project settings in Eclipse as an external JAR file.

The LoST server software also comes with a configuration file, similarly to the LoST client. It can be found in WebContent/WEB-INF/config/config.xml and an example LoST server configuration is shown in Listing A.4. Two different databases can be used to store civic and geodetic location information, which is not utilized in the example configuration because both point to the same database server. For LoST servers different modes of operations are available and can be set via the $<$ mode $>$ element in the config.xml file. These modes are based on the roles defined in RFC 5582 [55]. The following modes can be set:

Resolver A resolver is contacted by a seeker, interacts with forest guides, and then queries the appropriate tree. Resolvers may cache query results but do not source authoritative mapping information.

TreeLeaf Leaf nodes are nodes without children, and only maintain mappings.

TreeNode Tree nodes only maintain coverage regions. Coverage regions point to LoST servers while mapping data points to service URLs. Hence, the difference between coverage regions and mapping data is intentionally rather small and coverage regions are also implemented as LoST-compatible shapes enclosing contiguous geographic areas or by descriptors enumerating groups of civic locations. The primary difference is therefore to what entities the URLs point to.

ForestGuide Forest guides facilitate coordination among trees and they keep track of the coverage regions of all the trees for a service and a location profile. Forest guides only redirect to LoST mapping servers.

In the example configuration in Listing A.4 the LoST server operates in a TreeLeaf mode. The log file must be located in a writeable directory and the database indicated in the configuration file, postgis_lost in our example, must exist. More details about the database setup can be found in Section A.3.

Listing A.4: LoST Server Configuration Example

$<$ ?xml version="1.0" encoding="UTF-8"?> 


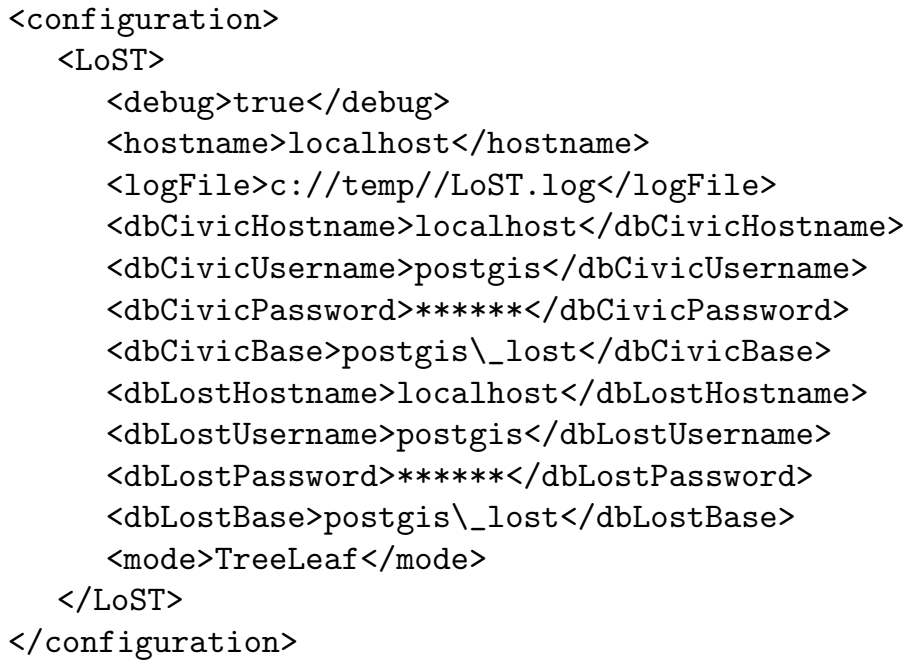

There are different ways to run server-side Java code; Java servlets is the technique utilized by this implementation. The LoST server implementations registers itself (via the Tomcat server) on a specific IP address, port number and at a specific URL path and waits for incoming POST messages. The @ WebServlet annotation is used to declare a servlet and in the LoST server implementation the code can be found in MainServlet.java file where the URL /StartServlet is registered. GET requests, on the other hand, will lead to an error (according to the LoST specification). An incoming LoST request will cause the LoST server to process the request according to its configured role. The prototype implementation is not designed for efficiency. It reads the configuration file and connects to the database with every request instead of reading the configuration once and maintaining it in memory as well as keeping the database connection open while the server is running. Hence, there is still potential for performance optimization. This has to be kept in mind when considering the performance analysis.

The LoST server implementation is split into several Java files, which can be grouped into four categories. These categories are (a) the main class, MainServlet.java, containing code executed when a request is received, (b) code handling specific LoST queries, (c) code implementing functionality needed by different LoST server roles, and (d) support functionality. Not relevant to this description is the test class LoSTClient.java.

The code for handling specific LoST requests can be found in the following Java classes:

FindService This class implements parsing of the findService and findServiceByLocation queries and produces the response message. Both geodetic and civic location information are supported. 
GetServiceBoundary This class implements parsing of the getServiceBoundary request. The getServiceBoundary request contains a value in the key attribute, which is used to find the service boundary in the cache. This code makes use of the getServiceById() and getIdByKey() functions implemented in the Base class.

ListServices This class implements parsing of the listServices query and makes use of the database access function in the Base class via the listServices method. The listServices method crafts the response message from the Response class via the listServicesResponse method.

ListServicesByLocation This class implements the listServicesByLocation query and, similiarly to the ListServices class, it also creates the corresponding response by utilizing the listServicesByLocation method from the Base class.

ServiceBoundary The methods in this class construct the $<$ serviceBoundary $>$ element based on civic or geodetic location information provided via parameters. For geodetic location information the polygon is encoded following the recommendations provided in RFC 5222 and RFC 5491. Not supported is the encoding of holes in services boundaries, as described in RFC 5964 [146]. The methods in this class, namely createGeodetic2d and createCivic, are used by the FindServiceResponse method of the FindService class and by the getServiceBoundary method in the GetServiceBoundary class.

The code implementing functionality needed by different LoST server roles can be found in the following Java classes:

Resolver The Resolver class implements functionality to fetch mappings from cache, and if those are not present or outdated, then Forest Guides have to be contacted.

ForestGuide The ForestGuide class implements functionality to fetch coverage regions from the database (for civic and geodetic location information) and to redirect the request to the appropriate LoST mapping server.

TreeNode The TreeNode class implements functionality to interact with other Tree Nodes or with Leaf Nodes (in an interative or recursive query style).

ParseFirst The ParseFirst class implements functionality of a TreeLeaf and contains code for parsingf LoST requests based on the different types of requests available within the LoST specification.

The support functionality is found in the following Java classes:

Base This class is responsible for handling database access. This class does not only include code for establishing the connection to the PostgreSQL database using Java Database Connectivity (JDBC) but also constructs the SQL queries needed for retrieving the necessary information from the database. One example of an important SQL query 
used by findServiceByLocation is shown in Listing A.5.

Cache and CacheStruct These two classes implement cache management.

CommonRequestPattern This class parses the XML document to find the $<$ service $>$ element.

Config This class reads the XML-based configuration file but also offers functionality for event logging and for setting timestamps (used in logs and in LoST response messages).

ErrorContainer This class implements the error handling and generates LoST error response messages.

Response This class implements generic LoST response functionality, such as creating the $<$ mapping $>$, the $<$ serviceList $>$, $<$ locationValidation $>$, and the $<$ path $>$ elements.

The Base class contains code to construct SQL queries and the most important SQL query is to determine the record(s) where the emergency caller's location is within the service boundary of a PSAP. This point-in-polygon functionality is provided by ST_Intersects() provided by PostGIS. It takes two geometries and returns true if any part of those geometries is shared between the two. The ST_AsText() function converts the location data from the database native binary format into a textual display format and ST_GeomFromText() provides the conversion into the reverse direction. Listing A.5 shows this important code segment.

Listing A.5: Java Code for Constructing the findServiceByLocation SQL Query

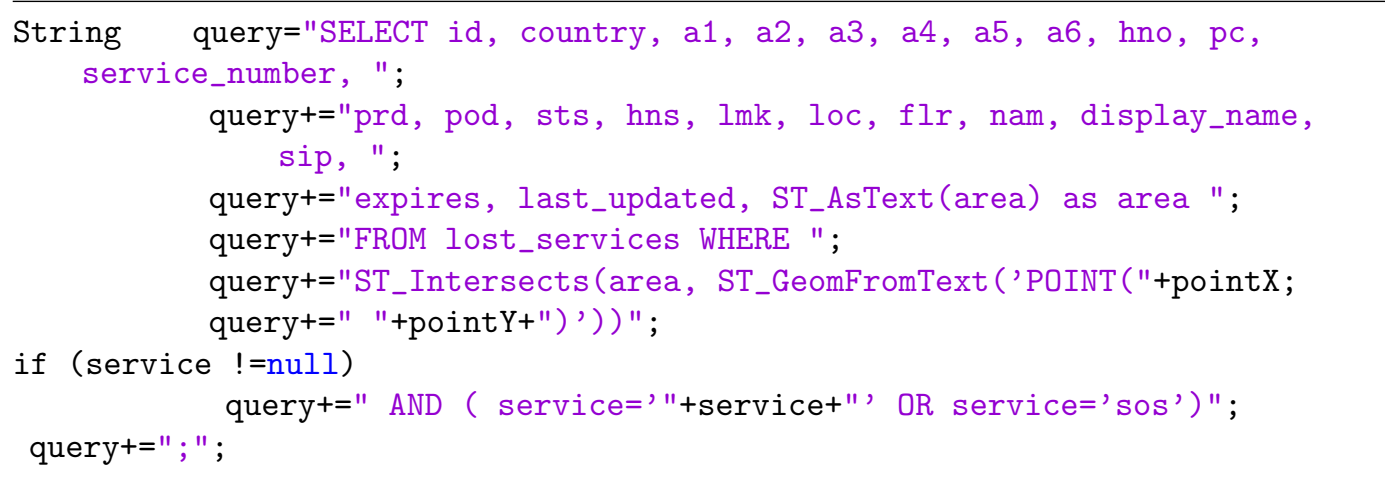

\section{A.3. Database}

The LoST server Java application issues queries against a number of tables whereby some of the tables are only used when the LoST server application operates in a specific role. The following tables are used for the different roles: 
Resolver The Resolver uses the resolver_cache and forest_guides tables. The former table is used for caching LoST responses while the latter table is used to obtain the list of LoST Forest Guides.

TreeLeaf The TreeLeave uses the lost_services and key_cache tables. The lost_services table is used to store authoritative mapping information. The key_cache table is used to store service boundary information when those are accessed by reference rather than by value.

TreeNode The TreeNode uses the tree_children_civic and tree_children_geodetic tables. These two tables are used to store coverage areas for civic and geodetic location information, respectively.

ForestGuide The Forest Guide uses the tree_roots_civic and tree_roots_geodetic tables. These two tables again store coverage areas.

Figure A.1 shows the database design with the tables graphically. 

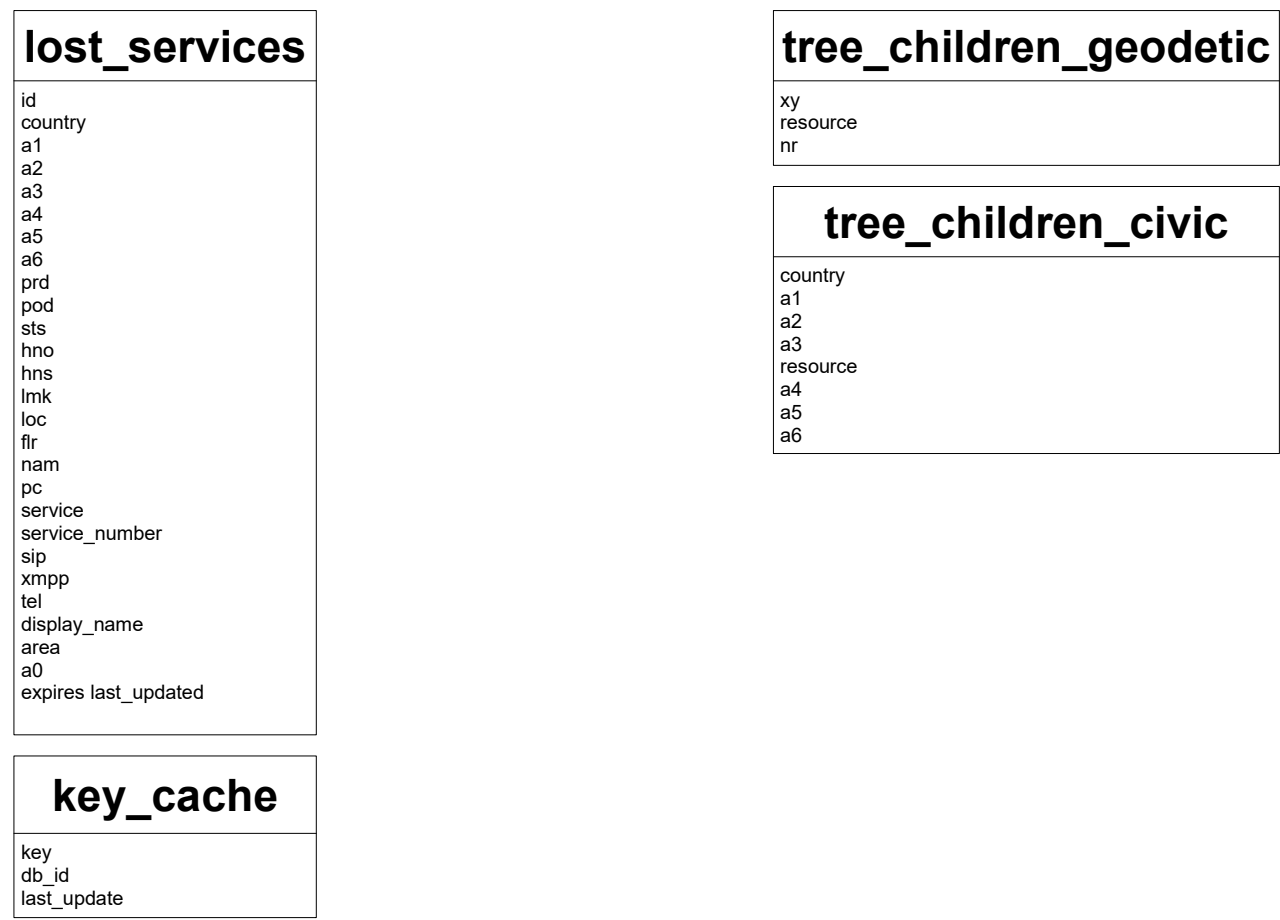

\begin{tabular}{|l|}
\hline resolver_cache \\
\hline $\begin{array}{l}\text { request } \\
\text { response } \\
\text { expires }\end{array}$ \\
\hline
\end{tabular}

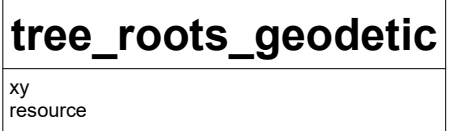

\section{forest_guides}

forest_guide

\begin{tabular}{|l|}
\hline \multicolumn{1}{|c|}{ tree_roots_civic } \\
\hline $\begin{array}{l}\text { country } \\
a 1 \\
a 2 \\
a 3 \\
a 3 \\
\text { resource }\end{array}$ \\
\hline
\end{tabular}

Figure A.1.: Database Design. 


\section{A.4. Performance Analysis}

While the main purpose of the LoST client and LoST server implementation was to verify correctness and completeness of the LoST specification, the performance was of interest to many. Although the code has not been optimized for performance it still provides an indication of the upper bounds. For a realistic analysis the most important aspect is thereby to select a country or region with many PSAP boundaries such that the point-in-polygon computation, which is considered to be one of the more expensive operation in a LoST-based deployment, can be evaludated. Unfortunately, it is difficult to obtain real-world PSAP boundaries, even for research purposes, because this type of information is still considered commerically valuable and a competitive advantage for those companies who obtained access to it. For practical reasons a simplifying assumption was made by assuming the PSAP boundaries correspond to the shapes of US counties. The shape files of U.S. counties are published by the US Census Bureau in the Topologically Integrated Geographic Encoding and Referencing system (TIGER) data set. The 2018 data set is available at https://www2. census.gov/geo/tiger/TIGER2018//COUNTY/ and has 3233 entries. The number of PSAPs in the US is roughly 6100; hence the TIGER county data set is a good approximation of the U.S., which has the most complex PSAP infrastructure of any country.

Figure A.2 shows a plot of the U.S. county database. The figure has been produced using the open-source cross-platform desktop geographic information system application QGIS, which is available for download at https://qgis.org, with the TIGER data retrieved from the PostgreSQL database. 


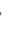

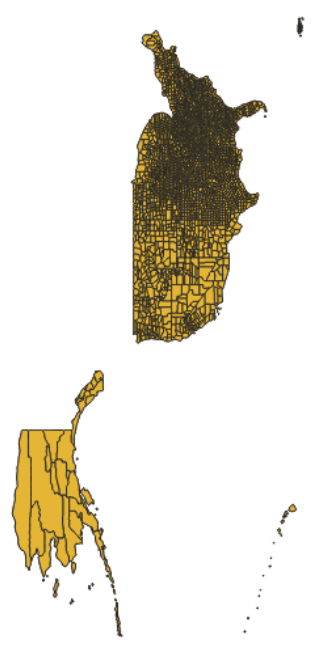

Figure A.2.: Plot of the U.S. TIGER County Dataset (2018). 
The TIGER data set is available in shapefiles, which cannot be imported directly into the Postgres database via the pgAdmin backup/restore tool. There are two techniques that can be used for importing the data set. First, it is possible to use the external PostGIS Shapefile Import/Export Manager tool. This tool can import the *.shp files found in the downloaded ZIP archives. Second, an automated, but considerable more complex, procedure for downloading and importing the TIGER data set is available in form of scripts. The latter approach uses the psql and the shp2pgsql command line tools. The next few paragraphs explain the automated data download and importing.

Assuming that the database has already been configured to use the extensions for postgis, fuzzystrmatch, postgis_tiger_geocoder, and address_standardizer the first step is to use the pgAdmin utility to configure the paths to various programs. This is, unsurprisingly, accomplished by modifying values in a specific field in the loader_platform and the loader_variables tables. It is easiest to use the pgAdmin utility to select these two tables in the tiger schema. By using the "View/Edit Data" function on the two tables showing all rows displays the current values. The values set for the declare_sect column have been set to the values in Listing A.6 on my system, which will have to be adjusted to each system depending on the US and the location of the binaries.

Listing A.6: Configuration Settings for the Loader Tables

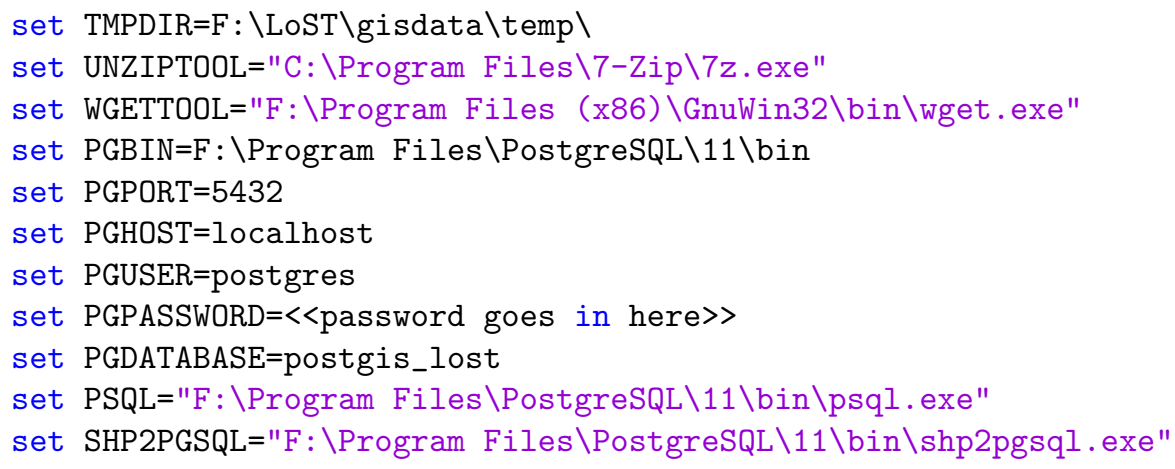

Once this configuration data has been saved, the configuration can be used to create a script, which will subsequently be used to perform the download and import. The command line to execute this script is shown in Listing A.7. The semantics of the command line parameters are described in the manual, which is available at https://www . postgresql. org/docs/current/app-psql.html.

Listing A.7: Command line to generate the so-called Nation-Script

$\mathrm{F}: \backslash$ LoST \gisdata>psql -U postgres -c "SELECT

Loader_Generate_Nation_Script('windows')" -d postgis_lost -tA > 
nation_script_load.bat

After successfully generating the nation_script_load.bat it can be executed as shown in Listing A.8.

Listing A.8: Running the Nation-Script File

$\mathrm{F}: \backslash$ LoST \gisdata>nation_script_load.bat

After a successful execution the data is imported into the indicated database and the correctness can be verified by counting the rows in the county and state tables. The rows in the county database should be 3233 and the number of rows in the state table should be 56 .

Note that the same technique can be used to retrieve additional data from the TIGER database to perform geocoding and reverse geocoding. Reverse geocoding is used to resolve a geodetic location into a civic address and geocoding performs the opposite. The commands shown in Listing A.9 are necessary to download and import detailed civic information of all states. Note, however, that the download of the detailed data will take both time (potentially in the order of days) and harddisk space.

Listing A.9: Running the State-Script File

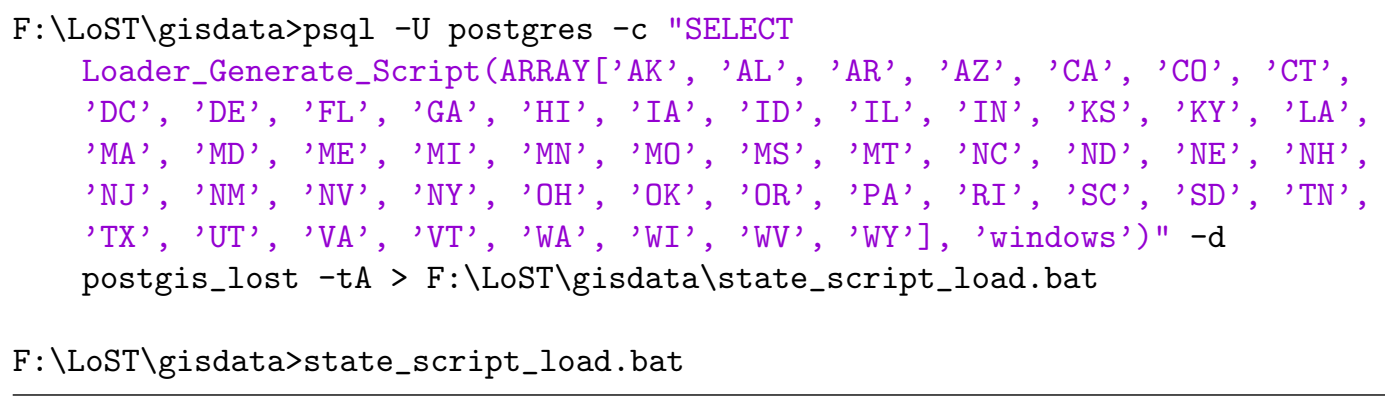

The database structure used by the TIGER imported data, of course, does not correspond to the structure the LoST server database uses. Hence, it is necessary to import relevant information from the TIGER database into the LoST database. Figure A.3 shows 10 rows added to the LoST database. 

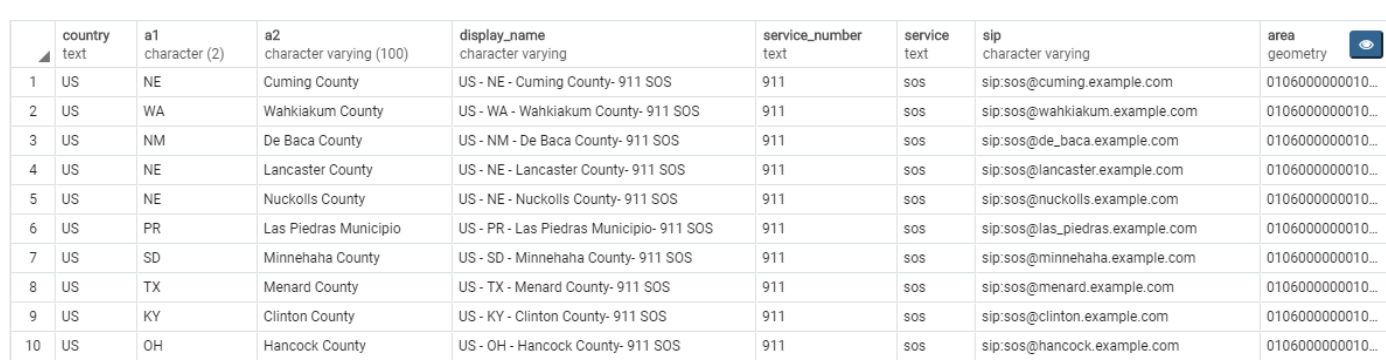

Figure A.3.: Example data to be inserted in LoST Database.

The SQL query in Listing A.11 has been used to add the data shown in Figure A.3 to the LoST database. Before it can be run, a separate table was created to perform a mapping between the abbreviations of state names with the numerical values used in the TIGER data set. The SQL script used for the import is shown in Listing A.10. While it is not stricly necessary to perform such a mapping, the use of state name abbreviations (such as 'CA' for California) greatly improves readability of the results and enables easier debugging for matching LoST requests against records in the database.

Listing A.10: SQL Code for Creating State Name Abbreviation Mapping

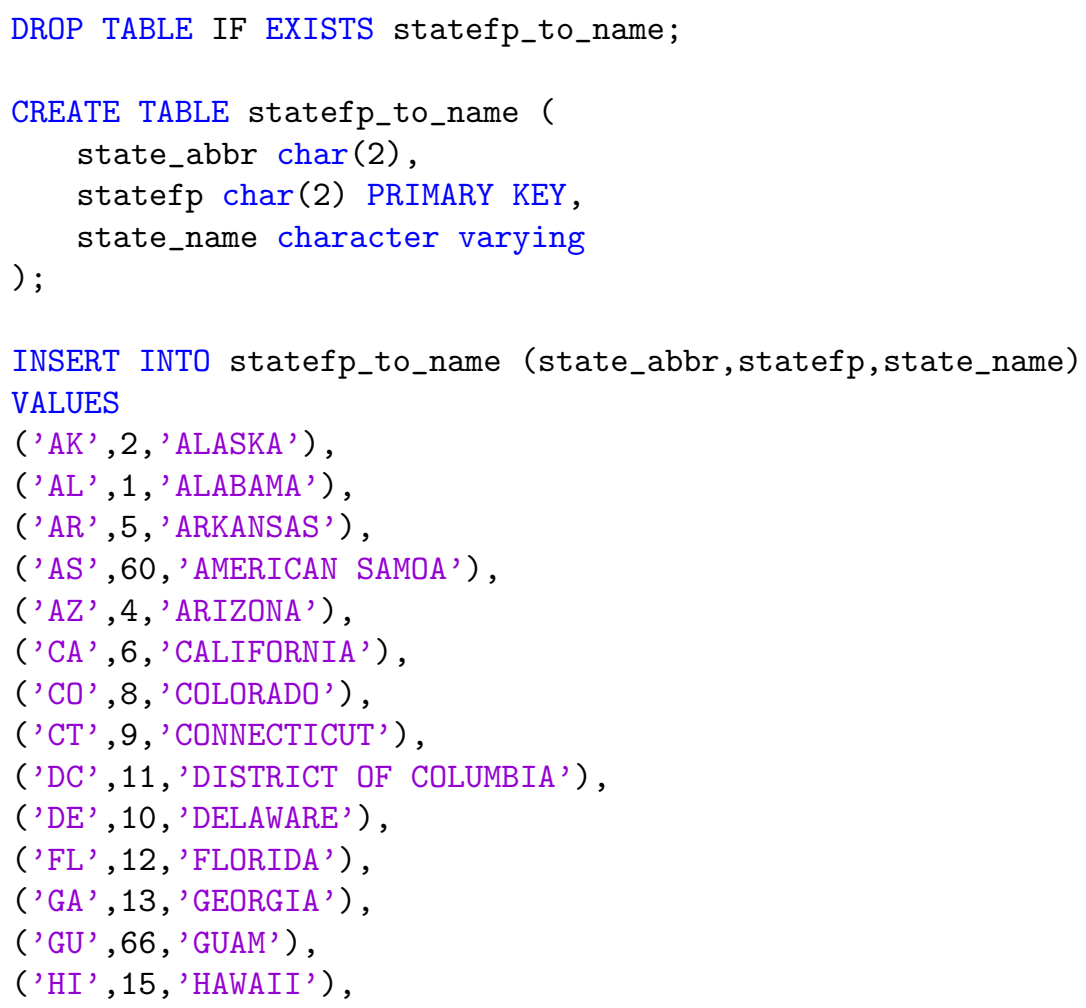




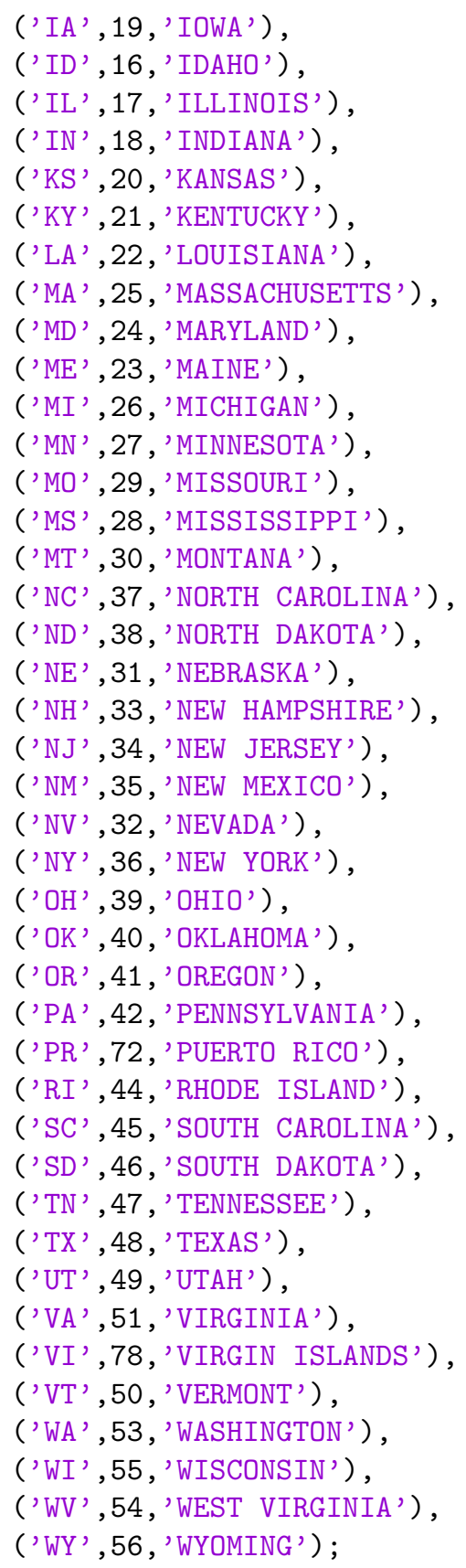

Listing A.11 shows the script that populates the lost_services table with data from the TIGER database. More specifically, the following data is copied: the country column, the 
A1 column (used for the top-level subdivision within a country, i.e. the state in the US), A2 column (used for the political district; the county in the US), the service_number column (which is set to 911 in the US), the service column (which uses the 'sos' service in the example only), the sip column (which is the PSAP URI to be used by the seeker for reaching out to a PSAP call taker over SIP), and the area column used to encode the service boundary. Note that the area column contains the polygon information encoded in a custom, PostgreSQL-specific binary format rather than the textual, GML-based representation shown earlier in LoST requests and responses. This is primarily done for performance reasons.

Listing A.11: SQL Script for Importing the TIGER Data into the LoST Database

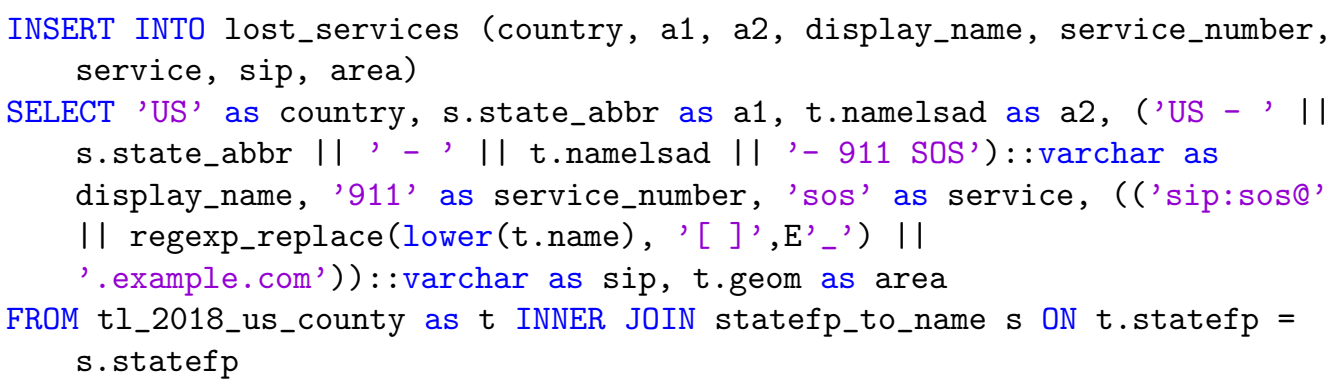

It is also useful to set the expires and last_updated fields of the records for correct operation of the LoST server application. Listing A.12 shows the SQL code.

Listing A.12: SQL Script for updating expires and last_updated

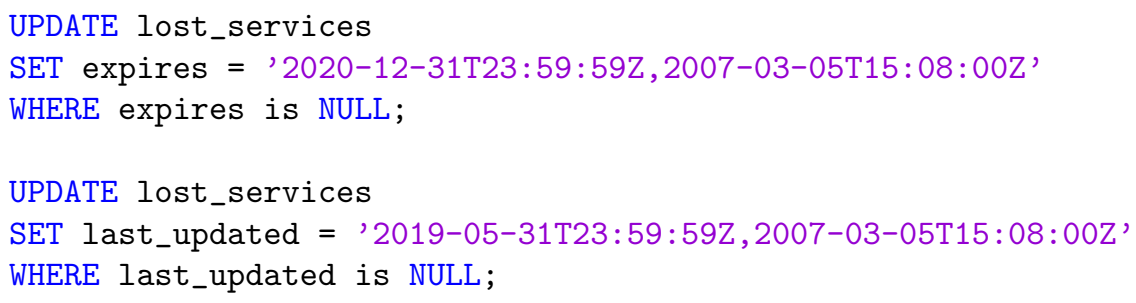

Once the example data is available in the LoST database, the next step is to construct example queries that will be used by the seeker against the LoST server. There have to be enough example queries to ensure that any anomalies caused by OS process scheduling, delay variation between the LoST Java application running on the Tomcat server and the PostgreSQL database, and varying latency on the network connection are averaged out. Additionally, the queries have to contain points somewhere in the US and cover all the counties. Furthermore, the location provided in two consecutive queries should not refer to locations directly next to each other to avoid any geolocation caching capabilities by 
the database because this is rather unlikely behavior with real emergency calls as well. Doing a 'linear scan' through points of the U.S. territory is therefore an unsuitable strategy. Unfortunately, there is no public data about the geographical distribution of emergency calls for selected regions available that could be re-used. Hence, any selection of example queries will at best be a rough approximation of real-world behavior.

The following approach had been selected:

- First, random points in and around the U.S. had been selected and tested against the database. When a hit was found the respective record was written into a database table called 'sample'.

- Second, the content of the 'sample' table was exported to a comma-separated values (csv) file.

- Third, a Python program was used to extract the data from the csv file and to execute LoST client calls. The results from those lookups was written into a text file for post-processing of the results.

To select example locations within the U.S. the built-in capability in PostgreSQL for random number generation with the random() function (in combination with the generate_series() function) was utilized. Listing A.13 shows the SQL command to create the table that holds the example queries. Note that the table contains more than just the $\mathrm{X} / \mathrm{Y}$ coordinates; information useful for debugging purposes, including the civic location information and the service boundary that matched the query, was added as well.

Listing A.13: SQL Script to create table to hold sample queries.

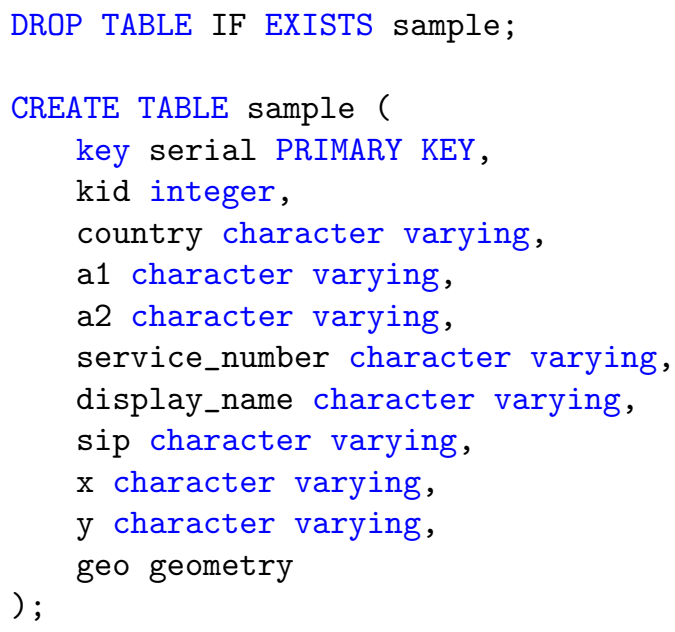


An SQL query covering the region of all U.S. counties, as shown in Listing A.14, is used to generate example queries, which are inserted into the 'sample' table.

Listing A.14: SQL Script to create sample queries.

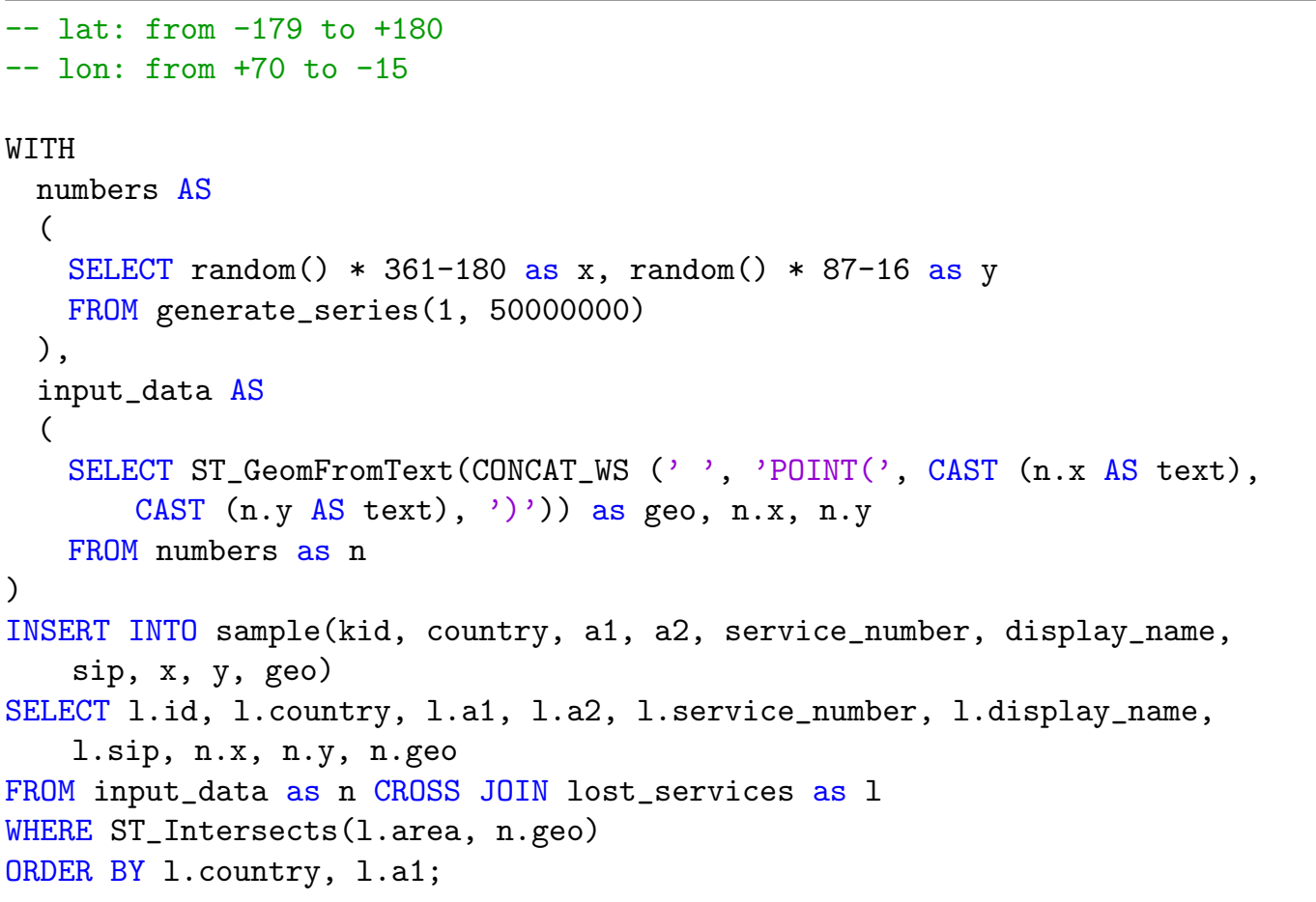

When identifying a region from which random $\mathrm{x} / \mathrm{y}$ coordinates are chosen it is useful to verify whether the expression is indeed correct. This can be accomplished by the SQL query shown in Listing A.15 where we use the $\min ()$ and $\max ()$ functions to determine whether the generated random values are indeed within the +70 to -15 range.

Listing A.15: Verifying correctness of the generated data.

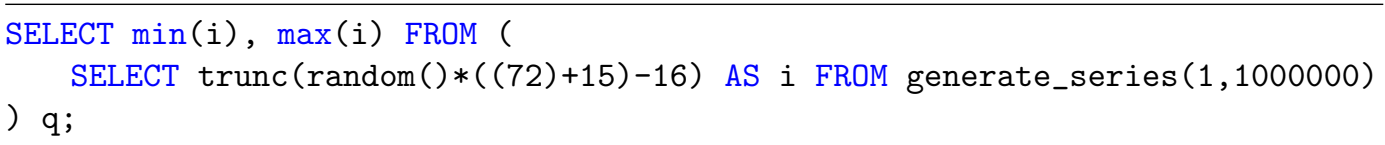

Plotting the example queries using the QGIS application, see Figure A.4, shows whether the sample queries have been correctly created and whether the entire region of the U.S. has been covered by the example queries. 
8

$\infty$

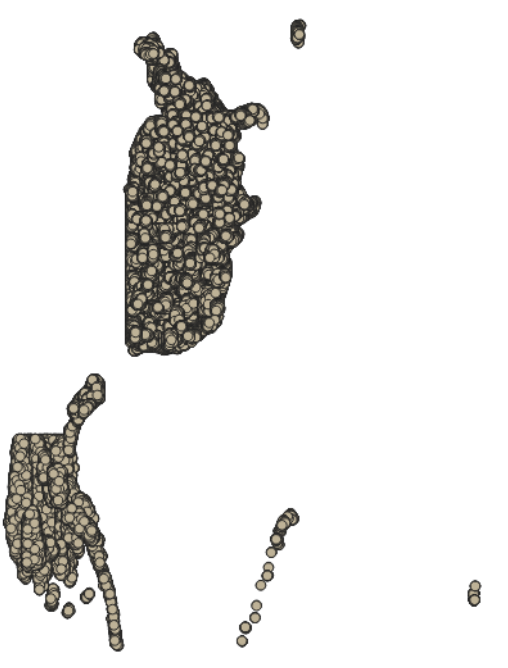

Figure A.4.: Plot of example queries. 
Next, we have to export the content of the 'sample' table to an csv file, which will be used for post-processing next. Listing A.16 shows the export commands.

Listing A.16: Exporting the example queries into a CSV file.

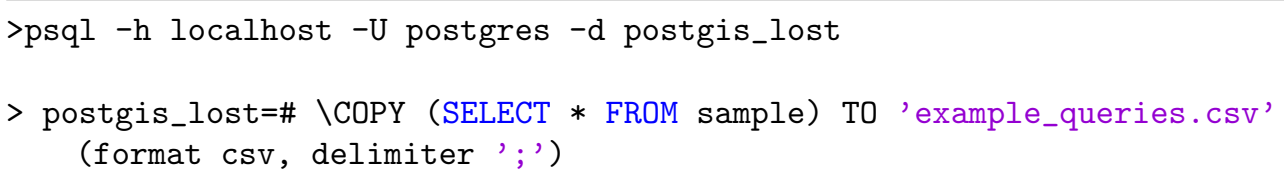

A small section of the CSV file is shown in Figure A.5. The most important data is in column 8 and 9 representing the $\mathrm{X}$ and $\mathrm{Y}$ coordinates of the query to be used.

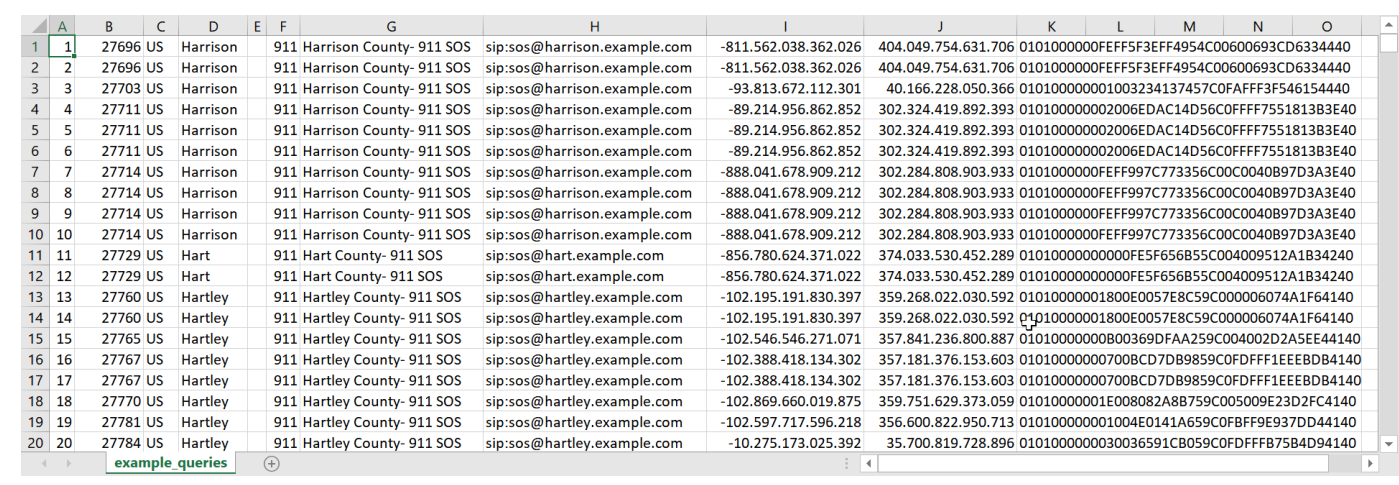

Figure A.5.: CSV file with example queries.

Next, the example_queries.csv is used as input to the Python script shown in Listing A.17. This short Python script reads each line of the CSV file and executes the LoST client program with the $\mathrm{X}$ and $\mathrm{Y}$ coordinates as command line parameters. The LoST client program has been compiled with the TIME_MEASUREMENT and the TIME_MEASUREMENT_SUMMARY C pre-processor directives enabled to produce timing data. This timing data is written to a log file for final processing, which contains three data points: the size of the LoST request (in bytes), the size of the LoST response (in bytes), and the execution time (in msec). The execution time includes processing the command line parameters to construct the LoST query until the time when the response has been received.

Listing A.17: Python Script to parse CSV file and execute LoST queries.

import csv

import subprocess 


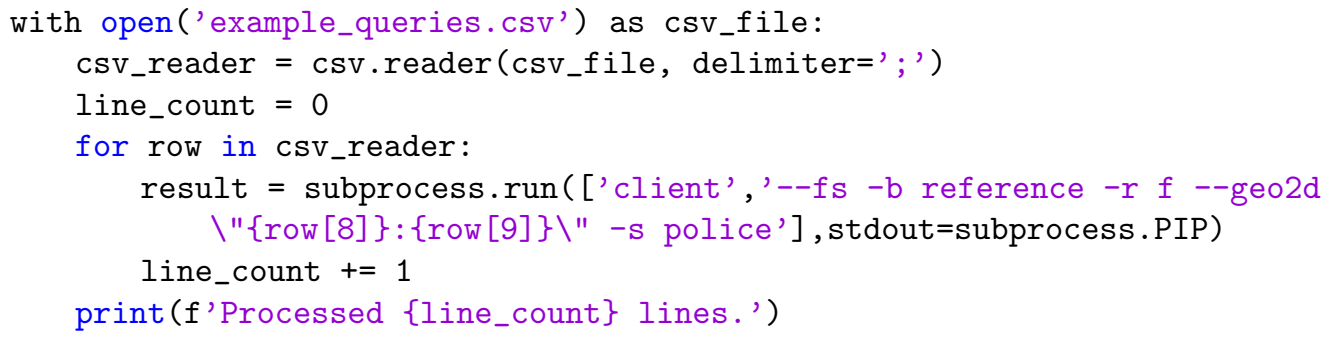

For this performance measurement the following assumptions were made:

- No TLS is used in this test setup. The overhead of TLS as a communication security solution is described in other parts of this thesis and, once the handshake has been completed, the overhead for symmetric key cryptography is minimal.

- Only geodetic location information is used because civic location lookups are much faster database operations and do not even require special extensions to database systems.

- Only a single location shape (namely a point) is used in queries because the data provided by the end device is supposed to be obtained via a GPS. Other location shapes, such as circles (which are able to express uncertainty) or arc bands (which are typically used in context of network-based location determination techniques) are not explored in this performance investigation.

- Caching capabilies are not explored in this performance analysis. The use of caching (by resolves as well as by seekers) would improve performance in a real-world scenario.

- Service boundares are transmitted by reference and subsequently not dereferenced. This leads to smaller messages transmitted over the wire and obtaining service boundaries is only useful when caching is utilized. Note also that the support for holes in service boundaries, as described in RFC 5964, is not implemented in this prototype.

- To reduce the impact of network latency all components, i.e. LoST clients executed sequentially from the Python script, LoST server as a Java application on the Tomcat server, and PostgreSQL database) were executed on the same machine.

For the test setup an Intel Xeon CPU E3-1231 v3 @ 3.40 GHz with 4 cores and 8 threads, with $16 \mathrm{~GB}$ of RAM and $8 \mathrm{MB}$ cache running Windows 10 Home in version 10.0.18362 was used. On the software side PgAdmin 4 in version 4.5 was used with PostgreSQL version 11.2 as well as Tomcat 9.0.19.

Figure A.6 shows the plot of the 550,000 query/response measurements graphically. The minimal roundtrip time (RTT) of the LoST request/response is $39 \mathrm{msec}$ and the maximum is $856 \mathrm{msec}$. The median is $55 \mathrm{msec}$ and the mean is $72.17 \mathrm{msec}$. The first and third quartile is $48 \mathrm{msec}$ and $65 \mathrm{msec}$, respectively. 


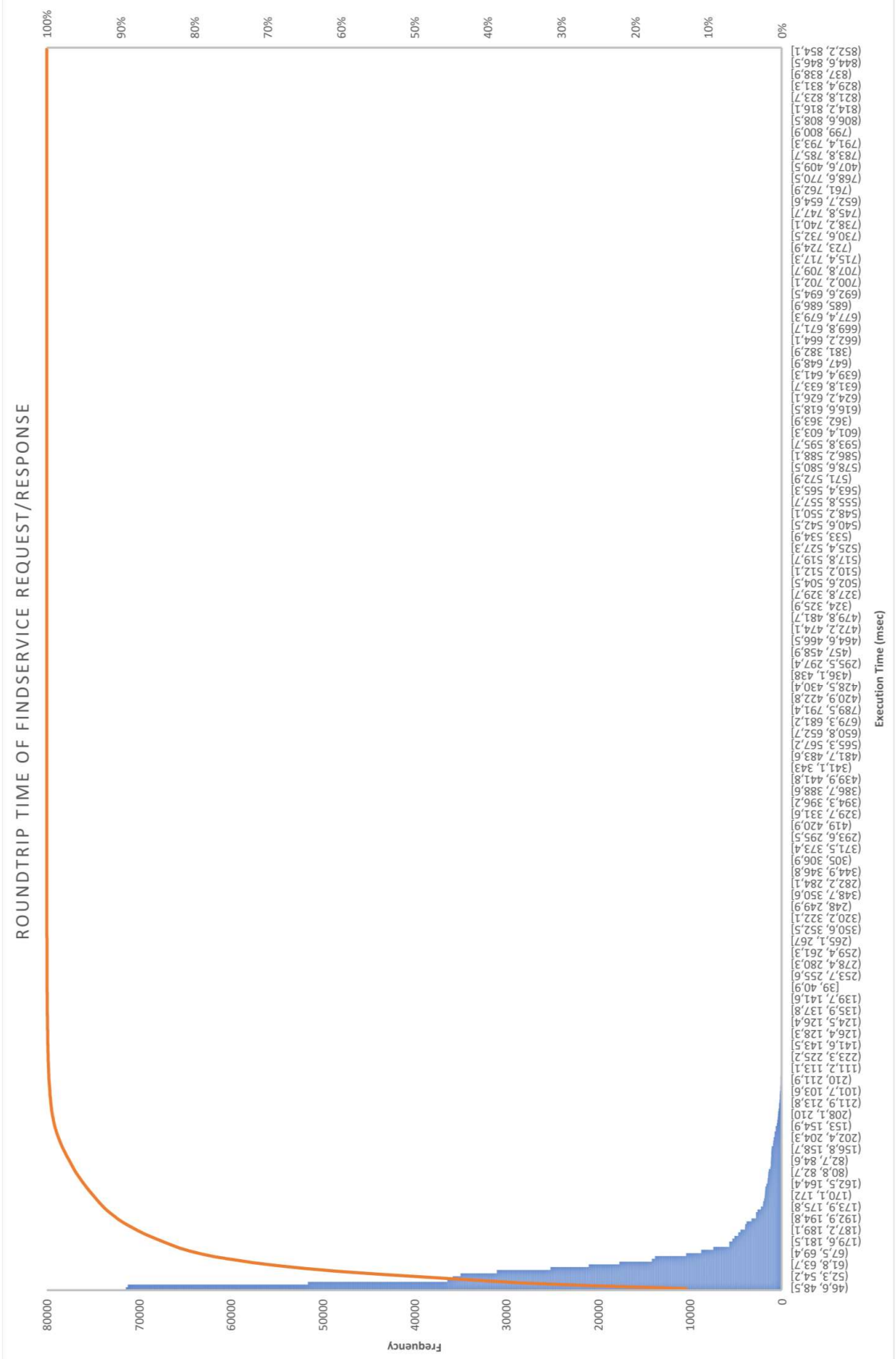

Figure A.6.: Roundtrip Time of findService Request/Response. 
As a conclusion, without special optimizations at the LoST server application nor at the database itself, without the use of caching at resolvers and seekers the RTT of a LoST interaction with a dataset taking the most complex PSAP infrastructure of any country into account the use of LoST at the end device is not only feasible but also possible without creating unnecessary delay in an emergency services situation. 


\section{Bibliography}

[1] NENA. National Emergency Number Association (NENA). URL: http://www . nena.org.

[2] S. L. Keoh, S. S. Kumar, and H. Tschofenig. Securing the internet of things: A standardization perspective. IEEE Internet of Things Journal, 1(3):265-275, June 2014.

[3] H. Tschofenig and E. Baccelli. Cyberphysical security for the masses: A survey of the internet protocol suite for internet of things security. IEEE Security Privacy, 17(5):47-57, Sep. 2019.

[4] K. Zandberg, K. Schleiser, F. Acosta, H. Tschofenig, and E. Baccelli. Secure firmware updates for constrained iot devices using open standards: A reality check. IEEE Access, PP:1-1, 052019.

[5] H. Schulzrinne and R. Marshall. Requirements for Emergency Context Resolution with Internet Technologies. RFC 5012, Internet Engineering Task Force, January 2008 .

[6] J. Peterson. A Presence-based GEOPRIV Location Object Format. RFC 4119, Internet Engineering Task Force, December 2005.

[7] H. Schulzrinne, H. Tschofenig, C. Holmberg, and M. Patel. Public Safety Answering Point (PSAP) Callback. RFC 7090, Internet Engineering Task Force, April 2014.

[8] H. Schulzrinne, S. McCann, G. Bajko, H. Tschofenig, and D. Kroeselberg. Extensions to the Emergency Services Architecture for Dealing With Unauthenticated and Unauthorized Devices. RFC 7406, Internet Engineering Task Force, December 2014.

[9] Randall Gellens, Brian Rosen, and Hannes Tschofenig. Next-Generation VehicleInitiated Emergency Calls. RFC 8148, May 2017.

[10] Brian Rosen, Henning Schulzrinne, Hannes Tschofenig, and Randall Gellens. Data- 
Only Emergency Calls. Internet-Draft draft-ietf-ecrit-data-only-ea-16, Internet Engineering Task Force, October 2018. Work in Progress.

[11] T. Taylor, H. Tschofenig, H. Schulzrinne, and M. Shanmugam. Security Threats and Requirements for Emergency Call Marking and Mapping. RFC 5069, Internet Engineering Task Force, January 2008.

[12] R. Marshall. Requirements for a Location-by-Reference Mechanism. RFC 5808, Internet Engineering Task Force, May 2010.

[13] H. Tschofenig and H. Schulzrinne. GEOPRIV Layer 7 Location Configuration Protocol: Problem Statement and Requirements. RFC 5687, Internet Engineering Task Force, March 2010.

[14] H. Schulzrinne, L. Liess, H. Tschofenig, B. Stark, and A. Kuett. Location Hiding: Problem Statement and Requirements. RFC 6444, Internet Engineering Task Force, January 2012.

[15] B. Rosen and J. Polk. Best Current Practice for Communications Services in Support of Emergency Calling. RFC 6881, Internet Engineering Task Force, March 2013.

[16] Wikipedia. National Emergency Number Association (NENA), November 2018.

[17] NENA. NENA Interim VoIP Architecture for Enhanced 9-1-1 Services (i2), Standard 08-001, December 2005.

[18] NENA. NENA Interim VoIP Architecture for Enhanced 9-1-1 Services (i2), Standard 08-001 v2, August 2010.

[19] H. Schulzrinne and Tschofenig. Internet Protocol-based Emergency Services. Wiley, August 2013.

[20] European Commission. Standardization Mandate to the European Standards Organisations (ESO) in Support of the Location Enhanced Emergency Call Service, M/493 EN, may 2011. URL: http://ec.europa.eu/growth/tools-databases/ mandates/index.cfm?fuseaction=select_attachments. download\&doc_ id $=820$.

[21] ETSI. Functional architecture to support European requirements on emergency caller location determination and transport, Final draft ETSI ES 203178 V1.0.0, apr 2014. URL: https://www .etsi.org/deliver/etsi_es/203100_203199/ 203178/01.00.00_50/es_203178v010000m.pdf. 
[22] ETSI. Protocol specifications for Emergency Service Caller Location determination and transport, Final draft ETSI ES 203283 V1.1.0, sep 2017. URL: https://www.etsi.org/deliver/etsi_es/203200_203299/ 203283/01.01.00_50/es_203283v010100m.pdf.

[23] M. Thomson and R. Bellis. Location Information Server (LIS) Discovery Using IP Addresses and Reverse DNS. RFC 7216, Internet Engineering Task Force, April 2014.

[24] M. Barnes. HTTP-Enabled Location Delivery (HELD). RFC 5985, Internet Engineering Task Force, September 2010.

[25] J. Winterbottom, M. Thomson, H. Tschofenig, and R. Barnes. Use of Device Identity in HTTP-Enabled Location Delivery (HELD). RFC 6155, Internet Engineering Task Force, March 2011.

[26] R. Bellis. Flow Identity Extension for HTTP-Enabled Location Delivery (HELD). RFC 6915, Internet Engineering Task Force, April 2013.

[27] J. Winterbottom, H. Tschofenig, and L. Liess. A routing request extension for the http-enabled location delivery (held) protocol. RFC 7840, RFC Editor, May 2016.

[28] H. Tschofenig, S. Decugis, J. Mahoney, and J. Korhonen. Diameter: New Generation AAA Protocol - Design, Practice, and Applications. Wiley, June 2019.

[29] ETSI. Network Technologies (NTECH); Network Attachment; e2 interface based on the DIAMETER protocol, ETSI ES 283035 V3.2.1, jan 2018. URL: https://www.etsi.org/deliver/etsi_es/283000_283099/ 283035/03.02.01_60/es_283035v030201p.pdf.

[30] H. Schulzrinne. Dynamic Host Configuration Protocol (DHCPv4 and DHCPv6) Option for Civic Addresses Configuration Information. RFC 4776, Internet Engineering Task Force, November 2006.

[31] J. Polk, J. Schnizlein, and M. Linsner. Dynamic Host Configuration Protocol Option for Coordinate-based Location Configuration Information. RFC 3825, Internet Engineering Task Force, July 2004.

[32] B. Rosen. Emergency Call Information in the Domain Name System, October 2005. draft-rosen-dns-sos-03.txt (expired), Internet Engineering Task Force.

[33] M. Mealling. Dynamic Delegation Discovery System (DDDS) Part One: The Com- 
prehensive DDDS. RFC 3401, Internet Engineering Task Force, October 2002.

[34] M. Mealling. Dynamic Delegation Discovery System (DDDS) Part Two: The Algorithm. RFC 3402, Internet Engineering Task Force, October 2002.

[35] M. Mealling. Dynamic Delegation Discovery System (DDDS) Part Three: The Domain Name System (DNS) Database. RFC 3403, Internet Engineering Task Force, October 2002.

[36] M. Mealling. Dynamic Delegation Discovery System (DDDS) Part Four: The Uniform Resource Identifiers (URI). RFC 3404, Internet Engineering Task Force, October 2002.

[37] J. Peterson, O. Kolkman, H. Tschofenig, and B. Aboba. Architectural Considerations on Application Features in the DNS. RFC 6950, Internet Engineering Task Force, October 2013.

[38] K. Wolf and A. Mayrhofer. Considerations for Civic Addresses in the Presence Information Data Format Location Object (PIDF-LO): Guidelines and IANA Registry Definition. RFC 5774, Internet Engineering Task Force, March 2010.

[39] J. Winterbottom, M. Thomson, R. Barnes, B. Rosen, and R. George. Specifying Civic Address Extensions in the Presence Information Data Format Location Object (PIDF-LO). RFC 6848, Internet Engineering Task Force, January 2013.

[40] J. Winterbottom, M. Thomson, and H. Tschofenig. GEOPRIV Presence Information Data Format Location Object (PIDF-LO) Usage Clarification, Considerations, and Recommendations. RFC 5491, Internet Engineering Task Force, March 2009.

[41] H. Schulzrinne and B. Rosen. Emergency Services for Internet Telephony Systems, October 2004. draft-schulzrinne-sipping-emergency-arch-02.txt (expired), Internet Engineering Task Force.

[42] H. Schulzrinne. A Uniform Resource Name (URN) for Emergency and Other WellKnown Services. RFC 5031, Internet Engineering Task Force, January 2008.

[43] H. Alvestrand. Overview: Real Time Protocols for Browser-based Applications. Internet-Draft draft-ietf-rtcweb-overview-19, Internet Engineering Task Force, 11 2017. Work in progress.

[44] P. Saint-Andre. Extensible Messaging and Presence Protocol (XMPP): Core. RFC 6120, Internet Engineering Task Force, March 2011. 
[45] B. Carpenter. Architectural Principles of the Internet. RFC 1958, Internet Engineering Task Force, June 1996.

[46] H. Tschofenig, B. Aboba, J. Peterson, and D. McPherson. Trends in Web Applications and the Implications on Standardization, May 2012. draft-tschofenig-poststandardization-02 (expired), Internet Engineering Task Force.

[47] EENA. Public Safety Answering Points Global Edition, December 2018. URL: https://eena.org/wp-content/uploads/2018-12-19_Abstract_Light. pdf (last accessed: Nov. 2019).

[48] Gary Machado. NG112 Transition Models - Implementation Activities, Version 2.0, July 2015. URL: https://eena.org/document/ ng112-transition-models-implementation-activities/ (last accessed: Nov. 2019).

[49] EENA. PSAP Directory: Supporting Inter-PSAP Communication Across National Border, June 2019. URL: https : //eena .org/document/psap-directory/ (last accessed: Nov. 2019).

[50] EENA. EENA Operations Document - Overload of calls, December 2012. URL: https://eena.org/document/overload-of-calls/, (last accessed: Nov. 2019).

[51] H. Schulzrinne and H. Tschofenig. Elements Based on the Location-to-Service Translation (LoST) Protocol. RFC 6739, Internet Engineering Task Force, October 2012.

[52] T. Hardie, A. Newton, H. Schulzrinne, and H. Tschofenig. LoST: A Location-toService Translation Protocol. RFC 5222, Internet Engineering Task Force, August 2008.

[53] C. Jennings, J. Peterson, and M. Watson. Private Extensions to the Session Initiation Protocol (SIP) for Asserted Identity within Trusted Networks. RFC 3325, Internet Engineering Task Force, November 2002.

[54] H. Tschofenig, H. Schulzrinne, and B. Aboba. Trustworthy Location. RFC 7378, Internet Engineering Task Force, December 2014.

[55] H. Schulzrinne. Location-to-URL Mapping Architecture and Framework. RFC 5582, Internet Engineering Task Force, September 2009. 
[56] H. Schulzrinne, J. Polk, and H. Tschofenig. Discovering Location-to-Service Translation (LoST) Servers Using the Dynamic Host Configuration Protocol (DHCP). RFC 5223, Internet Engineering Task Force, August 2008.

[57] B. Rosen, H. Schulzrinne, J. Polk, and A. Newton. Framework for Emergency Calling Using Internet Multimedia. RFC 6443, Internet Engineering Task Force, December 2011.

[58] B. Rosen. Dial String Parameter for the Session Initiation Protocol Uniform Resource Identifier. RFC 4967, Internet Engineering Task Force, July 2007.

[59] H. Schulzrinne. The tel URI for Telephone Numbers. RFC 3966, Internet Engineering Task Force, December 2004.

[60] M. Thomson and J. Winterbottom. Discovering the Local Location Information Server (LIS). RFC 5986, Internet Engineering Task Force, September 2010.

[61] R. Barnes, M. Thomson, J. Winterbottom, and H. Tschofenig. Location Configuration Extensions for Policy Management. RFC 7199, Internet Engineering Task Force, April 2014.

[62] REACH112. REsponding to All Citizens needing Help (REACH112) Project. URL: https://eena.org/eu-projects/reach-112/.

[63] R. Gellens and H. Tschofenig. Next-generation pan-european ecall. RFC 8147, RFC Editor, May 2017.

[64] R. Gellens, B. Rosen, H. Tschofenig, R. Marshall, and J. Winterbottom. Additional data related to an emergency call. RFC 7852, RFC Editor, July 2016.

[65] R. Barnes, M. Lepinski, A. Cooper, J. Morris, H. Tschofenig, and H. Schulzrinne. An Architecture for Location and Location Privacy in Internet Applications. RFC 6280, Internet Engineering Task Force, July 2011.

[66] J. Rosenberg, H. Schulzrinne, G. Camarillo, A. Johnston, J. Peterson, R. Sparks, M. Handley, and E. Schooler. SIP: Session Initiation Protocol. RFC 3261, Internet Engineering Task Force, June 2002.

[67] J. Polk, B. Rosen, and J. Peterson. Location Conveyance for the Session Initiation Protocol. RFC 6442, Internet Engineering Task Force, December 2011.

[68] Wikipedia. 'Swatting', November 2019. URL: https://en.wikipedia.org/ 
wiki/Swatting.

[69] EENA. False Emergency Calls, EENA Operations Document, Version 1.1, May 2011. URL: https://eena.org/document/false-emergency-calls/ (last accessed: Nov. 2019).

[70] H. Schulzrinne and J. Polk. Communications Resource Priority for the Session Initiation Protocol (SIP). RFC 4412, Internet Engineering Task Force, February 2006.

[71] L. Daigle and A. Newton. Domain-Based Application Service Location Using SRV RRs and the Dynamic Delegation Discovery Service (DDDS). RFC 3958, Internet Engineering Task Force, January 2005.

[72] P. Saint-Andre and J. Hodges. Representation and Verification of Domain-Based Application Service Identity within Internet Public Key Infrastructure Using X.509 (PKIX) Certificates in the Context of Transport Layer Security (TLS). RFC 6125, Internet Engineering Task Force, March 2011.

[73] D. Atkins and R. Austein. Threat Analysis of the Domain Name System (DNS). RFC 3833, Internet Engineering Task Force, August 2004.

[74] C. Rigney, S. Willens, A. Rubens, and W. Simpson. Remote Authentication Dial In User Service (RADIUS). RFC 2865, Internet Engineering Task Force, June 2000.

[75] V. Fajardo, J. Arkko, J. Loughney, and G. Zorn. Diameter Base Protocol. RFC 6733, Internet Engineering Task Force, October 2012.

[76] M. Thomson and J. Winterbottom. Using Device-Provided Location-Related Measurements in Location Configuration Protocols. RFC 7105, Internet Engineering Task Force, January 2014.

[77] J. Peterson, H. Schulzrinne, and H. Tschofenig. Secure Telephone Identity Problem Statement and Requirements. RFC 7340, Internet Engineering Task Force, September 2014.

[78] C. Mayville. 911 Swatters Cost Thousands, Endanger Lives, February 2009. URL: http://www.govtech.com/security/ 911-Swatters-Cost-Thousands-Endanger-Lives.html.

[79] FBI. Don't Make the Call - The New Phenomenon of 'Swatting', February 2008. URL: https://archives.fbi.gov/archives/news/stories/2008/ february/swatting020408, (last accessed: Nov. 2019). 
[80] Lucian Constantin. Vulnerability in embedded Web server exposes millions of routers to hacking, December 2014. URL: https://bit.1y/2RetduE.

[81] Lucian Constantin. At least 700K routers given to customers by ISPs can be hacked, March 2015. URL: http://www.computerworld.com/article/2899663/ at-least-700k-routers-given-to-customers-by-isps-can-be-hacked . html.

[82] L. Daigle. Domain-Based Application Service Location Using URIs and the Dynamic Delegation Discovery Service (DDDS). RFC 4848, Internet Engineering Task Force, April 2007.

[83] B. Aboba, L. Blunk, J. Vollbrecht, J. Carlson, and H. Levkowetz. Extensible Authentication Protocol (EAP). RFC 3748, Internet Engineering Task Force, June 2004.

[84] William E. Burr, Donna F. Dodson, Elaine M. Newton, Ray A. Perlner, W. Timothy Polk, Sarbari Gupta, and Emad A. Nabbus. NIST Special Publication 800-63-3: Electronic Authentication Guideline, August 2013. URL: http://nvlpubs .nist. gov/nistpubs/SpecialPublications/NIST .SP .800-63-3.pdf.

[85] R. Droms and W. Arbaugh. Authentication for DHCP Messages. RFC 3118, Internet Engineering Task Force, June 2001.

[86] E. Rescorla. HTTP Over TLS. RFC 2818, Internet Engineering Task Force, May 2000.

[87] P. Saint-Andre, M. Miller, and P. Hancke. Domain name associations (dna) in the extensible messaging and presence protocol (xmpp). RFC 7712, RFC Editor, November 2015 .

[88] M. Miller and P. Saint-Andre. Pkix over secure http (posh). RFC 7711, RFC Editor, November 2015.

[89] Y. Sheffer, R. Holz, and P. Saint-Andre. Recommendations for Secure Use of Transport Layer Security (TLS) and Datagram Transport Layer Security (DTLS). RFC 7525, Internet Engineering Task Force, May 2015.

[90] M. Handley, V. Jacobson, and C. Perkins. SDP: Session Description Protocol. RFC 4566, Internet Engineering Task Force, July 2006.

[91] J. Peterson and C. Jennings. Enhancements for Authenticated Identity Management in the Session Initiation Protocol (SIP). RFC 4474, Internet Engineering Task Force, 
August 2006.

[92] E. Rescorla and N. Modadugu. Datagram Transport Layer Security Version 1.2. RFC 6347, Internet Engineering Task Force, January 2012.

[93] Alissa Cooper, Hannes Tschofenig, Jon Peterson, and Bernard Aboba. Secure call origin identification. Internet-Draft draft-cooper-iab-secure-origin-00, IETF Secretariat, November 2012.

[94] J. Peterson, C. Jennings, E. Rescorla, and C. Wendt. Authenticated identity management in the session initiation protocol (sip). RFC 8224, RFC Editor, February 2018.

[95] C. Wendt and J. Peterson. Passport: Personal assertion token. RFC 8225, RFC Editor, February 2018.

[96] J. Fischl, H. Tschofenig, and E. Rescorla. Framework for Establishing a Secure Realtime Transport Protocol (SRTP) Security Context Using Datagram Transport Layer Security (DTLS). RFC 5763, Internet Engineering Task Force, May 2010.

[97] D. McGrew and E. Rescorla. Datagram Transport Layer Security (DTLS) Extension to Establish Keys for the Secure Real-time Transport Protocol (SRTP). RFC 5764, Internet Engineering Task Force, May 2010.

[98] M. Jones, J. Bradley, and N. Sakimura. JSON Web Token (JWT). RFC 7519, Internet Engineering Task Force, May 2015.

[99] D. Hardt. The OAuth 2.0 Authorization Framework. RFC 6749, Internet Engineering Task Force, October 2012.

[100] J. Peterson and S. Turner. Secure telephone identity credentials: Certificates. RFC 8226, RFC Editor, February 2018.

[101] H. Schulzrinne, S. Casner, R. Frederick, and V. Jacobson. RTP: A Transport Protocol for Real-Time Applications. RFC 3550, Internet Engineering Task Force, July 2003.

[102] J. Lazzaro. Framing Real-time Transport Protocol (RTP) and RTP Control Protocol (RTCP) Packets over Connection-Oriented Transport. RFC 4571, Internet Engineering Task Force, July 2006.

[103] D. Yon and G. Camarillo. TCP-Based Media Transport in the Session Description Protocol (SDP). RFC 4145, Internet Engineering Task Force, September 2005. 
[104] J. Lennox. Connection-Oriented Media Transport over the Transport Layer Security (TLS) Protocol in the Session Description Protocol (SDP). RFC 4572, Internet Engineering Task Force, July 2006.

[105] M. Baugher, D. McGrew, M. Naslund, E. Carrara, and K. Norrman. The Secure Real-time Transport Protocol (SRTP). RFC 3711, Internet Engineering Task Force, March 2004.

[106] D. Wing, S. Fries, H. Tschofenig, and F. Audet. Requirements and Analysis of Media Security Management Protocols. RFC 5479, Internet Engineering Task Force, April 2009.

[107] F. Andreasen, M. Baugher, and D. Wing. Session Description Protocol (SDP) Security Descriptions for Media Streams. RFC 4568, Internet Engineering Task Force, July 2006.

[108] J. Franks, P. Hallam-Baker, J. Hostetler, S. Lawrence, P. Leach, A. Luotonen, and L. Stewart. HTTP Authentication: Basic and Digest Access Authentication. RFC 2617, Internet Engineering Task Force, June 1999.

[109] T. Dierks and E. Rescorla. The Transport Layer Security (TLS) Protocol Version 1.2. RFC 5246, Internet Engineering Task Force, August 2008.

[110] C. Bormann, M. Ersue, and A. Keranen. Terminology for Constrained-Node Networks. RFC 7228, Internet Engineering Task Force, May 2014.

[111] Oliver Hahm, Emmanuel Baccelli, Hauke Petersen, and Nicolas Tsiftes. Operating Systems for Low-End Devices in the Internet of Things: a Survey. IEEE Internet of Things Journal, 3(5):720-734, October 2016.

[112] European Union Agency For Network and Information Security (ENISA). Baseline Security Recommendations for IoT in the context of Critical Information Infrastructures, November 2017. URL: https://www .enisa. europa.eu/publications/ baseline-security-recommendations-for-iot.

[113] H. Tschofenig, J. Arkko, D. Thaler, and D. McPherson. Architectural Considerations in Smart Object Networking. RFC 7452, Internet Engineering Task Force, March 2015.

[114] E. Rescorla and B. Korver. Guidelines for Writing RFC Text on Security Considerations. RFC 3552, Internet Engineering Task Force, July 2003. 
[115] A. Cooper, H. Tschofenig, B. Aboba, J. Peterson, J. Morris, M. Hansen, and R. Smith. Privacy Considerations for Internet Protocols. RFC 6973, Internet Engineering Task Force, July 2013.

[116] S. Farrell and H. Tschofenig. Pervasive Monitoring Is an Attack. RFC 7258, Internet Engineering Task Force, May 2014.

[117] Hannes Tschofenig and Thomas Fossati. Transport Layer Security (TLS) / Datagram Transport Layer Security (DTLS) Profiles for the Internet of Things. RFC 7925, July 2016.

[118] Eric Rescorla. The Transport Layer Security (TLS) Protocol Version 1.3. RFC 8446, August 2018.

[119] D. Taylor, T. Wu, N. Mavrogiannopoulos, and T. Perrin. Using the Secure Remote Password (SRP) Protocol for TLS Authentication. RFC 5054, Internet Engineering Task Force, November 2007.

[120] Robert Cragie and Feng Hao. Elliptic Curve J-PAKE Cipher Suites for Transport Layer Security (TLS). Internet-Draft draft-cragie-tls-ecjpake-01, Internet Engineering Task Force, June 2016. Work in Progress.

[121] H. Krawczyk and P. Eronen. HMAC-based Extract-and-Expand Key Derivation Function (HKDF). RFC 5869, Internet Engineering Task Force, May 2010.

[122] Eric Rescorla, Hannes Tschofenig, and Nagendra Modadugu. The Datagram Transport Layer Security (DTLS) Protocol Version 1.3. Internet-Draft draft-ietf-tls-dtls1333, Internet Engineering Task Force, October 2019. Work in Progress.

[123] Eric Rescorla, Hannes Tschofenig, and Thomas Fossati. Connection Identifiers for DTLS 1.2. Internet-Draft draft-ietf-tls-dtls-connection-id-07, Internet Engineering Task Force, October 2019. Work in Progress.

[124] G. Selander, J. Mattsson, F. Palombini, and L. Seitz. Object security for constrained restful environments (oscore). RFC 8613, RFC Editor, July 2019.

[125] Jim Schaad. CBOR Object Signing and Encryption (COSE). RFC 8152, July 2017.

[126] Angelo P. Castellani, Salvatore Loreto, Akbar Rahman, Thomas Fossati, and Esko Dijk. Guidelines for Mapping Implementations: HTTP to the Constrained Application Protocol (CoAP). RFC 8075, February 2017. 
[127] Ludwig Seitz, Göran Selander, Erik Wahlstroem, Samuel Erdtman, and Hannes Tschofenig. Authentication and Authorization for Constrained Environments (ACE) using the OAuth 2.0 Framework (ACE-OAuth). Internet-Draft draft-ietf-ace-oauthauthz-25, Internet Engineering Task Force, October 2019. Work in Progress.

[128] Francesca Palombini, Ludwig Seitz, Göran Selander, and Martin Gunnarsson. OSCORE profile of the Authentication and Authorization for Constrained Environments Framework. Internet-Draft draft-ietf-ace-oscore-profile-08, Internet Engineering Task Force, July 2019. Work in Progress.

[129] M. Jones and J. Hildebrand. JSON Web Encryption (JWE). RFC 7516, Internet Engineering Task Force, May 2015.

[130] M. Jones, J. Bradley, and N. Sakimura. JSON Web Signature (JWS). RFC 7515, Internet Engineering Task Force, May 2015.

[131] Michael Jones, Erik Wahlstroem, Samuel Erdtman, and Hannes Tschofenig. CBOR Web Token (CWT). RFC 8392, May 2018.

[132] Michael Jones, John Bradley, and Hannes Tschofenig. Proof-of-Possession Key Semantics for JSON Web Tokens (JWTs). RFC 7800, April 2016.

[133] Michael Jones, Ludwig Seitz, Göran Selander, Samuel Erdtman, and Hannes Tschofenig. Proof-of-Possession Key Semantics for CBOR Web Tokens (CWTs). Internet-Draft draft-ietf-ace-cwt-proof-of-possession-11, Internet Engineering Task Force, October 2019. Work in Progress.

[134] Giridhar Mandyam, Laurence Lundblade, Laurence Lundblade, Miguel Ballesteros, and Jeremy O'Donoghue. The Entity Attestation Token (EAT). Internet-Draft draftietf-rats-eat-01, Internet Engineering Task Force, July 2019. Work in Progress.

[135] H. Tschofenig and N. Smith. Credential Management for Internet of Things Devices. Technical report, Internet Protocol for Smart Objects (IPSO) Alliance, December 2017. URL: https://www. omaspecworks .org/wp-content/uploads/ 2018/03/IPSO-IoT-Credential-Management_Final.pdf.

[136] Open Mobile Alliance. Lightweight Machine to Machine Technical Specification v1.1. Technical report, Open Mobile Alliance, July 2018. URL: http://www . openmobilealliance.org/release/LightweightM2M/V1_1-20180710-A/.

[137] Behcet Sarikaya, Mohit Sethi, and Dan Garcia-Carillo. Secure IoT Bootstrapping: A Survey. Internet-Draft draft-sarikaya-t2trg-sbootstrapping-07, Internet Engineering 
Task Force, July 2019. Work in Progress.

[138] E. Lear, R. Droms, and D. Romascanu. Manufacturer usage description specification. RFC 8520, RFC Editor, March 2019.

[139] Brendan Moran, Milosch Meriac, Hannes Tschofenig, and David Brown. A Firmware Update Architecture for Internet of Things Devices. Internet-Draft draftietf-suit-architecture-07, Internet Engineering Task Force, October 2019. Work in Progress.

[140] Brendan Moran, Hannes Tschofenig, and Henk Birkholz. SUIT CBOR manifest serialisation format. Internet-Draft draft-ietf-suit-manifest-01, Internet Engineering Task Force, October 2019. Work in Progress.

[141] Mingliang Pei, Andrew Atyeo, Nick Cook, Minho Yoo, and Hannes Tschofenig. The Open Trust Protocol (OTrP). Internet-Draft draft-ietf-teep-opentrustprotocol-03, Internet Engineering Task Force, May 2019. Work in Progress.

[142] Mingliang Pei, Hannes Tschofenig, Dave Wheeler, Andrew Atyeo, and Dapeng Liu. Trusted Execution Environment Provisioning (TEEP) Architecture. Internet-Draft draft-ietf-teep-architecture-03, Internet Engineering Task Force, July 2019. Work in Progress.

[143] Hannes Tschofenig. Security Risks in Next-Generation Emergency Services. Communications of the ACM, 54(11):23-25, 2011. http://doi.acm.org/10.1145/2018396.2018405.

[144] E. Trevisani and A. Vitaletti. Cell-ID Location Technique, Limits and Benefits: An Experimental Study. In WMCSA '04: Proceedings of the Sixth IEEE Workshop on Mobile Computing Systems and Applications (WMCSA'04), pages 51-60, 2004.

[145] EENA. Caller Location in Support of Emergency Services, EENA Operations Document, Version 2.0, November 2014. URL: https://eena.org/document/ caller-location-in-support-of-emergency-services-updated/, (last accessed: Nov. 2019).

[146] J. Winterbottom and M. Thomson. Specifying Holes in Location-to-Service Translation (LoST) Service Boundaries. RFC 5964, Internet Engineering Task Force, August 2010 . 